\title{
Herpetological Monitoring and Assessment on the Trinity River, Trinity County, California-Final Report
}

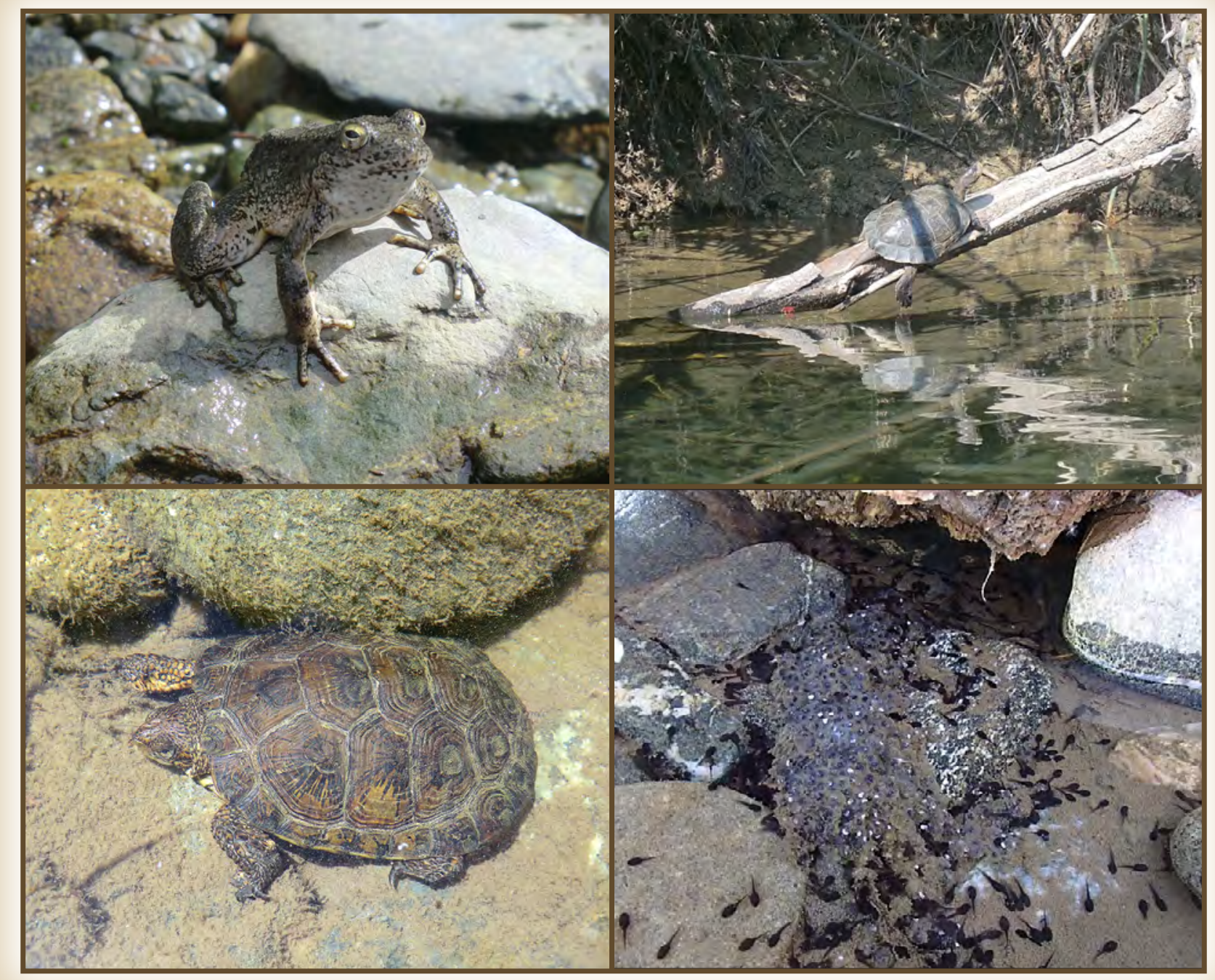

Open-File Report 2016-1089 
Cover: Photographs of adult Foothill yellow-legged frog (Rana boylii) (top left); basking adult western pond turtle (Actinemys marmorata) (top right); juvenile western pond turtle (bottom left); and Foothill yellow-legged frog egg mass with embryo mortality (bottom right). Photographs by Don Ashton, U.S. Geological Survey, June 2014. 


\section{Herpetological Monitoring and Assessment on the Trinity River, Trinity County, California-Final Report}

By Melissa L. Snover and Michael J. Adams

Prepared in cooperation with the Trinity River Restoration Program

Open-File Report 2016-1089

U.S. Department of the Interior

U.S. Geological Survey 


\section{U.S. Department of the Interior \\ SALLY JEWELL, Secretary}

\section{U.S. Geological Survey \\ Suzette M. Kimball, Director}

U.S. Geological Survey, Reston, Virginia: 2016

For more information on the USGS—-the Federal source for science about the Earth,

its natural and living resources, natural hazards, and the environment-visit

http://www.usgs.gov/ or call 1-888-ASK-USGS (1-888-275-8747).

For an overview of USGS information products, including maps, imagery, and publications, visit http://www.usgs.gov/pubprod/.

Any use of trade, firm, or product names is for descriptive purposes only and does not imply endorsement by the U.S. Government.

Although this information product, for the most part, is in the public domain, it also may contain copyrighted materials as noted in the text. Permission to reproduce copyrighted items must be secured from the copyright owner.

Suggested citation:

Snover, M.L., and Adams, M.J., 2016, Herpetological monitoring and assessment on the Trinity River, Trinity County, California_Final report: U.S. Geological Survey Open-File Report 2016-1089, 93 p., http://dx.doi.org/10.3133/ofr20161089.

ISSN 2331-1258 (online) 


\section{Contents}

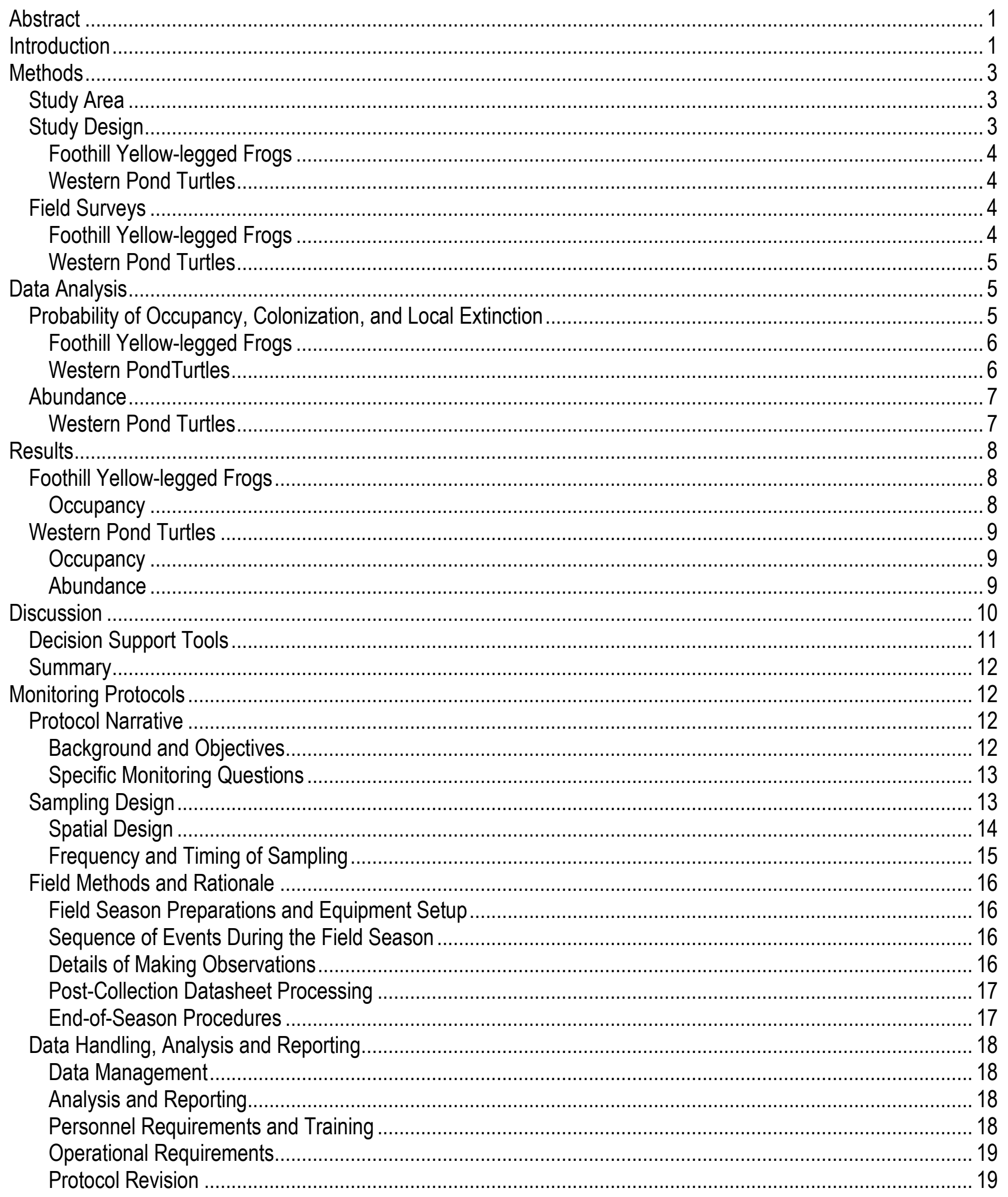


Standard Operation Procedures for Monitoring Herpetological Species ……..................................................... 20

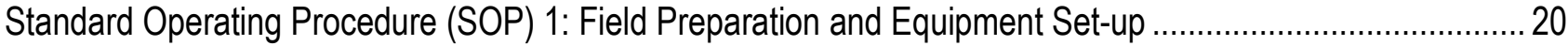

Standard Operating Procedure (SOP) 2: Personnel and Training ............................................................ 23

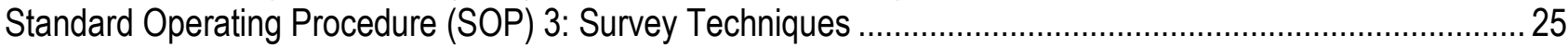

Standard Operating Procedure (SOP) 4: Data Management ………...................................................... 30

Standard Operating Procedure (SOP) 5: Spatial Data Management........................................................ 31

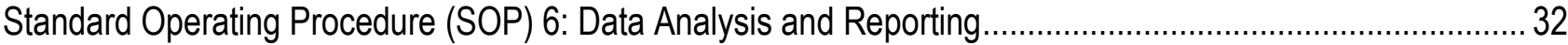

Standard Operating Procedure (SOP) 7: Revising the Protocol .............................................................. 34

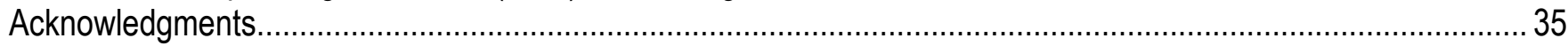

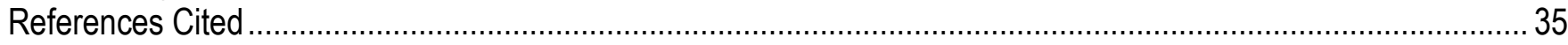

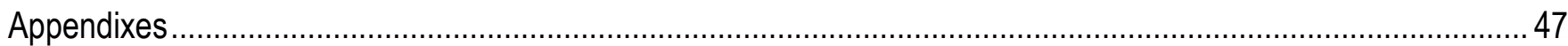

Appendix A. Example of Field Schedule Based on the 2015 Season............................................................... 47

Appendix B. U.S. Geological Survey Hygiene Protocol for Control of Disease Transmission Between Amphibian

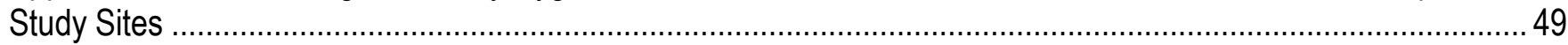

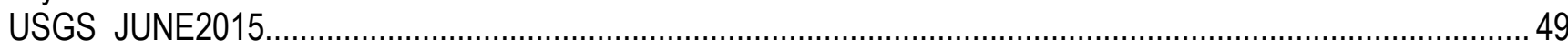

Appendix C. Metadata for Database: Foothill Yellow-legged Frogs ................................................................. 51

Appendix D. Metadata for Database: Western Pond Turtles ………...........................................................56

Appendix E. Generalized Linear Random Tessellation Stratified Sampling Protocol (GRTS) Panel Identification .. 60

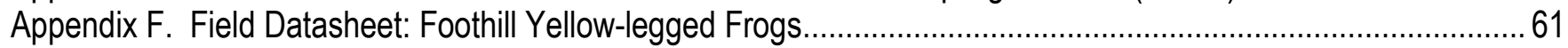

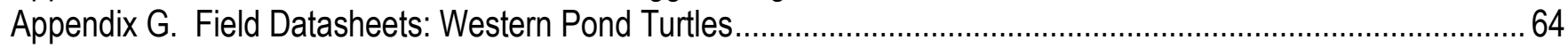

Appendix H. Pond Location and Information for Western Pond Turtle Surveys .................................................... 79

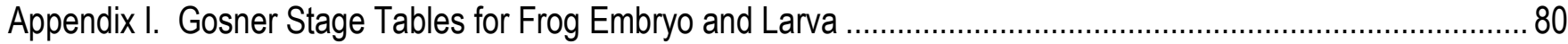

Appendix J. Input Data Structure for R Script: Foothill Yellow-Legged Frogs ...................................................... 81

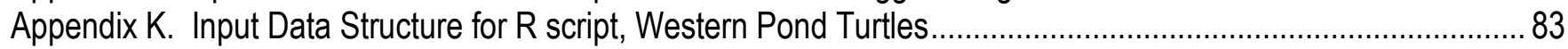

Appendix L. R Script for Foothill Yellow-Legged Frog Occupancy Model .............................................................. 85

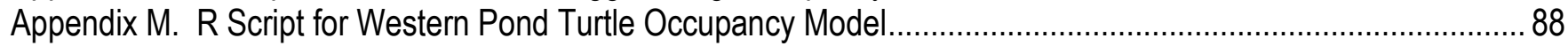

Appendix N. R Script for Western Pond Turtle Abundance Model.................................................................... 92

\section{Figures}

Figure 1. Study area for herpetological surveys on main stem Trinity River from the Lewiston Dam to the confluence with the North Fork Trinity River, Trinity County, California

Figure 2. Summary of habitat characteristics for 808 basking western pond turtle (Actinemys marmorata) sightings along the Trinity River, Trinity County, California, 2013-15.

Figure 3. Conceptual model for the links between management decisions and population processes for foothill yellow-legged frogs (Rana boylii) on the Trinity River, Trinity County, California.

Figure 4. Conceptual model for the links between management decisions and population processes for western pond turtles (Actinemys marmorata) on the Trinity River, Trinity County, California..

Figure 5. Sample sites for foothill yellow-legged frogs (Rana boylii) from the Lewiston Dam to the confluence with the North Fork Trinity River, Trinity County, California, 2013-15.

Figure 6. Sample sites for western pond turtle (Actinemys marmorata) surveys from the fish weir to the confluence with the North Fork Trinity River, Trinity River, Trinity County, California, 2013-15 ....

Figure 7. Initiation and duration of foothill yellow-legged frog (Rana boylii) breeding activity as it relates to flow and water temperature, Trinity River, Trinity County, California, 2006-09. 


\section{Tables}

Table 1. Multi-season occupancy models describing the probability that a site was occupied by breeding foothill yellow-legged frogs (Rana boylii), Trinity River, Trinity County, California, 2013-15.....

Table 2. Occupancy model results of yellow-legged frogs (Rana boylii) and western pond turtles (Actinemys marmorata), Trinity River, Trinity County, California, 2013-15 using data from all generalized linear random tessellation stratified sampling (GRTS) panels and data from five-panel GRTS only. ....

Table 3. Multi-season occupancy models describing the probability that a site was occupied by basking western pond turtles (Actinemys marmorata), Trinity River, Trinity County, California, 2013-15.

Table 4. Multi-season occupancy models describing the probability that a site was occupied by basking western pond turtles (Actinemys marmorata) observed in ponds adjacent to the Trinity River, Trinity County, California, 2013-15.

\section{Conversion Factors}

International System of Units Inch/Pound

\begin{tabular}{ccc}
\hline Multiply & By & To obtain \\
\hline & Flow rate & \\
\hline cubic foot per second $\left(\mathrm{ft}^{3} / \mathrm{s}\right)$ & 0.02832 & cubic meter per second $\left(\mathrm{m}^{3} / \mathrm{s}\right)$ \\
\hline
\end{tabular}

Inch/Pound to International System of Units

\begin{tabular}{lcl}
\hline & Bultiply & \multicolumn{1}{l}{ To obtain } \\
\hline centimeter $(\mathrm{cm})$ & Length & \\
meter $(\mathrm{m})$ & 0.3937 & inch (in.) \\
\hline & 1.094 & yard $(\mathrm{yd})$ \\
\hline cubic meter per second $\left(\mathrm{m}^{3} / \mathrm{s}\right)$ & Flow rate & \\
\hline
\end{tabular}

Temperature in degrees Celsius $\left({ }^{\circ} \mathrm{C}\right)$ may be converted to degrees Fahrenheit $\left({ }^{\circ} \mathrm{F}\right)$ as ${ }^{\circ} \mathrm{F}=\left(1.8 \times{ }^{\circ} \mathrm{C}\right)+32$.

Temperature in degrees Fahrenheit $\left({ }^{\circ} \mathrm{F}\right)$ may be converted to degrees Celsius $\left({ }^{\circ} \mathrm{C}\right)$ as ${ }^{\circ} \mathrm{C}=\left({ }^{\circ} \mathrm{F}-32\right) / 1.8$.

\section{Datums}

Vertical coordinate information is referenced to North American Vertical Datum of 1983 (NAVD 83)].

Horizontal coordinate information is referenced to North American Datum of 1983 (NAD 83)].

Altitude, as used in this report, refers to distance above the vertical datum. 
This page left intentionally blank 


\title{
Herpetological Monitoring and Assessment on the Trinity River, Trinity County, California-Final Report
}

\author{
By Melissa L. Snover and Michael J. Adams
}

\begin{abstract}
The primary goal of the Trinity River Restoration Program is to rehabilitate the fisheries on the dam-controlled Trinity River. However, maintaining and enhancing other wildlife populations through the restoration initiative is also a key objective. Foothill yellow-legged frogs (Rana boylii) and western pond turtles (Actinemys marmorata) have been identified as important herpetological species on which to focus monitoring efforts due to their status as California state-listed species of concern and potential listing on the U.S. Endangered Species List. We developed and implemented a monitoring strategy for these species specific to the Trinity River with the objectives of establishing baseline values for probabilities of site occupancy, colonization, and local extinction; identifying site characteristics that correlate with the probability of extinction; and estimating overall trends in abundance. Our 3-year study suggests that foothill yellow-legged frogs declined in the probability of site occupancy. Conversely, our results suggest that western pond turtles increased in both abundance and the probability of site occupancy. The short length of our study period makes it difficult to draw firm conclusions, but these results provide much-needed baseline data. Further monitoring and directed studies are required to assess how habitat changes and management decisions relate to the status and trend of these species over the long term.
\end{abstract}

\section{Introduction}

The construction of two dams, an upper (Trinity Dam) and a lower (Lewiston Dam), on the main stem of the Trinity River, Trinity County, California, was completed in 1964. The result of this action, combined with subsequent flooding events and water control actions, was a severely modified channel, with an estimated loss of 80-90 percent of fish habitat for native salmon and steelhead populations (U.S. Department of the Interior, 2000). Efforts to rehabilitate the river began in 1980 with the passage of the Trinity River Stream Rectification Act and continue today with efforts being led by the interagency Trinity River Restoration Program (TRRP; U.S. Department of the Interior, 2000). Although the TRRP is primarily focused on rehabilitating the fisheries, the program uses an ecosystembased approach, with a complementary aim of protecting and restoring other wildlife populations along the river. Consequently, a considerable amount of research and monitoring has been done on the herpetofauna associated with the river, especially the foothill yellow-legged frog (Rana boylii) and the western pond turtle (Actinemys marmorata), both of which are listed as California Species of Special Concern (Jennings and 
Hayes, 1994). The U.S. Fish and Wildlife Service was recently petitioned to consider both of these species for listing under the Endangered Species Act, and the 90-day findings indicated that the petitions may be warranted. Status reviews are therefore underway for both species (U.S. Fish and Wildlife Service, 2015a, 2015b).

The foothill yellow-legged frogs and western pond turtles were monitored on the Trinity River by the U.S. Forest Service from 1991 to 1994 (Lind and others, 1996; Reese and Welsh, 1998a, 1998b) and from 2004 to 2009 (Wheeler and others, 2013, 2014; Ashton and others, 2015; Snover and others, 2015). For foothill yellow-legged frogs, Lind and others (1996) monitored variable numbers of sites (gravel bars) each year before and after peak spring flows and documented losses of egg masses deposited prior to peak flows. The objectives of the monitoring from 2004 to 2009 were to compare breeding phenology among several Trinity River tributaries (Wheeler and others, 2013, 2014). For western pond turtles, both monitoring periods consisted of capture-mark-recapture studies conducted on limited sections of the Trinity and South Fork Trinity Rivers to compare size and age distribution, abundance, and reproductive efforts between the rivers (Reese and Welsh, 1998a, 1998b; Ashton and others, 2010, 2015; Snover and others, 2015).

These studies have provided valuable information on aspects of the populations as they compare to unregulated waterways and changes in demographics between the sampling timeframes. In particular, the foothill yellow-legged frog work highlights how the timing of dam releases affects egg mass survival (Lind and others, 1996); and how the cooler water temperatures of the Trinity River delays breeding, hatching, and metamorphosis compared to unregulated waterways (Wheeler and others, 2013, 2014). Comparisons of western pond turtles between decades and between regulated and unregulated rivers have highlighted reduced growth rates, delayed maturity, and smaller size at maturity for Trinity River turtles surveyed in the 2000s (Ashton and others, 2015; Snover and others, 2015). This information has provided an understanding of the links between dam operations and restoration activities and the population processes for both species.

One of the key ongoing issues for both of these species is the management decision to maintain uncharacteristically low water temperatures compared to surrounding water bodies. The purpose of the temperature goals is to mitigate for the loss of natural cool-water fish habitats caused by the dams, and advance the rehabilitation of the fisheries (U.S. Fish and Wildlife Service and Hoopa Valley Tribe, 1999). These cool-water habitats provide a refuge for large fish, minimizing disease and large-scale dieoffs from disease and decreased dissolved oxygen. Another key management decision that relates specifically to foothill yellow-legged frogs is the volume and timing of the spring release from the dam. Annual decisions are made that classify the water year on a gradient from critically dry to extremely wet, and Department of the Interior (2000) specifies the volume of the spring dam release based on this classification, with increasing volumes along the critically dry to extremely wet spectrum. These spring releases coincide with oviposition in the foothill yellow-legged frog, and they can result in scouring if egg masses are deposited prior to the release, or in dessication if egg masses are deposited during the descending limb of the release (Lind and others, 1996). These observed effects of water management on frog and turtle populations necessitated the development of a comprehensive monitoring protocol for these sensitive species. 
Our objectives for developing monitoring protocols for the foothill yellow-legged frog and western pond turtle populations on the Trinity River were to: (1) estimate site-level probability of occupancy, colonization, and extinction rates for both species; (2) examine how site characteristics and habitat changes, especially as they relate to restoration decisions, correlate with the probability of local extinction; and (3) estimate the overall trend in abundance for western pond turtles. A long-term goal for this project is to inform management decisions as they relate to water releases from the dam and habitat modifications. Hence, we also consider how these objectives can inform the development of decision support tools.

\section{Methods}

\section{Study Area}

The primary study area was the main stem Trinity River from the Lewiston Dam to the confluence with the North Fork Trinity River in Trinity County, California (fig. 1). For foothill yellowlegged frogs, the study area also included perennial waterways that feed into the primary study area (fig. 1). For western pond turtles, the study area included perennial ponds within $500 \mathrm{~m}$ of the centerline of the primary study area (fig. 1).

\section{Study Design}

Our monitoring approach was based on a study design developed for fisheries monitoring by the Trinity River Restoration Program partners. Specifically, they developed a study design using a generalized linear random tessellation stratified sampling protocol (GRTS) with a rotating panel revisit design (California Department of Fish and Game and others, 2010). A GRTS sample site was defined as a 400-m segment of the Trinity River centerline at a flow rate of 5,000 $\mathrm{ft}^{3} / \mathrm{s}$. The rotating panel design consisted of 5 panels, and each panel was composed of 16 GRTS sample sites. Each year, 2 panels were sampled, and in subsequent years 1 of the panels from the previous year was repeated, taking 5 years to sample all panels. These 5 panels represented 50 percent of the sample sites on the Trinity River between the Lewiston Dam and the confluence with the North Fork Trinity river (see California Department of Fish and Game and others [2010] for additional details on this sampling protocol and rotating panel design).

This study design is being used in the fish habitat studies, and has been recommended for application to other studies conducted on the river to enhance opportunities for integrating data (California Department of Fish and Game and others, 2010). Given the small population sizes and sparse distribution of our focal species, we anticipated that the rotating panel design would provide limited information on distribution and abundance of amphibians and reptiles. In addition, our monitoring work was relatively quick in comparison to the fish habitat studies; we were able to conduct monitoring in up to 30 GRTS panels per day for foothill yellow-legged frogs and as many as 60 panels per day for western pond turtles. Hence, though we were consistent with the defined segments of the GRTS design, we did not adopt the rotating panel revisit design. In our initial surveys, we included all GRTS sample sites for both species, that is, all sample sites between Steel Bridge and the confluence with the North Fork Trinity River for foothill yellow-legged frogs, and between Lewiston and the confluence with the North Fork Trinity River for western pond turtles. In our analysis we contrasted the results of surveys including all GRTS units with results where we limited the surveys to the 5 panel GRTS units (50 percent of the sample universe). 


\section{Foothill Yellow-legged Frogs}

In the first year, we surveyed the entire study area for signs of foothill yellow-legged frog breeding juveniles or adults. Within each GRTS sample site, we surveyed all potential breeding habitat. Previous studies have identified the characteristics of breeding habitat for foothill yellow-legged frogs on the Trinity River (Lind and others, 1996; Wheeler and others, 2014). These characteristics include gravel bars with shallow water, slow flow, little canopy cover, and temperatures that generally exceed an average of $12^{\circ} \mathrm{C}$ on a daily basis. The temperature requirements for breeding were not achieved upstream of the Steel Bridge River Access, hence, in the subsequent 2 years, main stem surveys were limited to downstream of the Steel Bridge River Access because of the lack of thermal habitat upstream of this location (fig. 1). Of all of the perennial tributaries surveyed in 2013 (fig. 1), eight were determined to contain breeding habitat, and in 2014 and 2015 only these tributaries were monitored. One additional tributary, Sheridan Creek, might have breeding habitat, but access was denied by the landowners.

\section{Western Pond Turtles}

On the Trinity River, we surveyed all GRTS sample sites from the fish weir at the Lewiston Dam to the confluence with the North Fork Trinity River (fig. 1). For off-channel habitats, 35 ponds were initially identified within $500 \mathrm{~m}$ of the centerline of the main stem Trinity River primary study site from previous studies (Sloan, 2012) or from the National Hydrography Dataset (http://www.nhd.usgs.gov). Two additional ponds were identified in the field. Of the 37 ponds, 20 were surveyed for western pond turtles (fig. 1). The remaining ponds were not surveyed either because they were dry $(\mathrm{N}=11)$, could not be located $(\mathrm{N}=2)$, or because we were unable to contact the landowners or were denied access $(\mathrm{N}=4)$.

\section{Field Surveys}

\section{Foothill Yellow-legged Frogs}

A two-person crew surveyed gravel bars with shallow, slow-moving water within the primary study area. One person walked the shoreline slightly ahead of the second person, who walked below the water line, and the observations of both crew members counted as one survey. Location and counts of foothill yellow-legged frog egg masses, larvae, juveniles, and adults were recorded. Two or three repeat visits were conducted at all surveyed gravel bars between the Steel Bridge River Access and the confluence with the North Fork Trinity River. For tributaries that contained flowing water, we surveyed all gravel bar habitat within the first $400 \mathrm{~m}$ from the confluence with the Trinity River; one exception was Oregon Gulch, where access past $120 \mathrm{~m}$ was denied by landowners.

Survey specific variables were collected at each site, including air temperature ("air"), water temperature of the main channel ("water"), wind ("wind") and sky conditions ("weather"; see appendix $\mathrm{C}$ for the wind and weather classifications). At each gravel bar, off-channel habitats were noted, including side channels (connected to the main channel at the top and bottom), inlets (connected to the main channel but not flowing), and backwaters (disconnected from the main channel). Habitat variables for each GRTS sample site were summarized as a linear measure of 
the shoreline with habitat ("length"), measured from a track line recorded with a global positioning system receiver (GPS); the percent of habitat associated with the main channel ("pctmc", contrasted with off-channel habitats); and the distance from the midpoint of the GRTS sample site to the closest upstream occupied tributary (“distUP”).

\section{Western Pond Turtles}

A two-person crew in separate kayaks floated the primary study area, proceeding as slowly as possible. Each crew member was assigned a river bank (river right or river left) and scanned the bank downriver using binoculars. All basking western pond turtles were counted and locations recorded. Surveys of the primary study area were repeated three times. When deemed safe, all side channels and back waters immediately adjacent to the main channel also were surveyed. Surveys of ponds differed slightly from the primary study area. Ponds were scanned for at least 5 minutes by a single observer, and observations were repeated three times, alternating observers and completing all surveys in a single visit. All observed western pond turtles were counted and recorded.

Habitat variables were collected for each GRTS sample site and pond, and again at each basking turtle sighting along the river. These variables included dominant vegetation ("veg"), substrate and morphology of the river bank ("bank"), amount of basking habitat ("bask"), water velocity ("vel"), depth ("depth"), each metric collected for the left and right banks of river, and distance downstream of the dam ("distance"; see appendix D for further information on the habitat variables). In addition, the same survey variables collected for the frog surveys also were collected within each GRTS sample site for western pond turtles. One change in the protocol for 2015 was to determine the habitat variables for each GRTS on a separate float, whereas in 2013 and 2014 the habitat variables were collected in conjunction with the first survey. This change may affect the probability of detection for the first survey.

\section{Data Analysis}

\section{Probability of Occupancy, Colonization, and Local Extinction}

We used the function "colext" from the R package "unmarked" (v0.11-0) in R (v3.2.1) to estimate probabilities for initial occupancy, colonization, and local extinction, considering that not all individuals or egg masses present at a site will be detected and that the probability of detection $(p)$ is likely to vary (Fiske and Chandler, 2011; R Core Team, 2015). The function "colext" fits the multiseason occupancy model of MacKenzie and others (2003). We used this model to investigate how the probability of local extinction $(\varepsilon)$ was related to site characteristics and to derive estimates of site occupancy $(\psi)$. With only 3 years of data, we anticipated difficulties estimating the transition probabilities and therefore used a simplifying assumption that colonization $(\gamma)$ was constant across years and sites for all analyses. 


\section{Foothill Yellow-legged Frogs}

For this analysis, occupied GRTS units were those with eggs or larvae of foothill yellow-legged frogs, indicating active breeding. We used the global model:

$\psi_{1}$ (distUP,gravel), $\varepsilon$ (distUP,gravel,pctmc), $\gamma(),$.$p (year,date, water,wind,weather)$

For detection, we included the covariate "year" so that change in the probability of detecting foothill yellow-legged frogs that were present would not bias estimates of trend in occupancy. We also considered additional effects on $p$ including date, because changes in larval behavior and abundance over the season might affect $p$; water temperature, which might affect larval activity; wind, because this can obscure the water surface and affect detection; and sky conditions, which might affect visibility.

We included the closest upstream inhabited tributary ("distUP") as a covariate of $\psi$ because these locations may serve as sources of breeding adults. We also included the amount of breeding habitat within sites ("gravel") as a covariate of $\psi$. Off-channel habitats offer different conditions from the main channel, including higher water temperature, slower flows, and potentially higher risks of desiccation, hence we included the percent of habitat characterized as main channel as a site covariate.

To estimate initial occupancy $\left(\psi_{1}\right), \gamma$, and $\varepsilon$, we fit a colext model with the following covariates of each parameter:

$$
\psi_{1} \text { (dist,gravel), } \varepsilon(.), \gamma(.), p(\text { year })
$$

To compare the effects of site characteristics on $\varepsilon$, we fit the following variations on the $\varepsilon$ portion of the model leaving the $\psi, \gamma$, and $p$ portions as shown in previous model:

$$
\varepsilon \text { (gravel,dist,pctmc), } \varepsilon(\text { dist }), \varepsilon(\text { gravel }), \varepsilon(\text { pctmc), } \varepsilon(.) \text {. }
$$

We also included a null model, $\psi_{1}(),. \varepsilon(),. \gamma(),. p($.$) , in our model comparisons. Probabilities of$ occupancy in subsequent years were derived from the estimates of $\psi_{1}, \gamma$, and $\varepsilon$, and we used a nonparametric bootstrap to obtain the standard errors for $\psi_{2}$ and $\psi_{3}$ (appendix L).

We ran the occupancy analysis with data from all GRTS sites and repeated the analysis with data from the five-panel GRTS only to determine if the subset of GRTS sampling units from the five panels provides adequate spatial coverage to estimate the occupancy parameters.

Western Pond Turtles model:

Occupied sites are GRTS sample sites that had basking western pond turtles. We used the global

$\psi($ bask, distance), $\varepsilon($ bank, bask, vel, distance), $\gamma()$,

$p$ (bank, bask, vel, distance, date, date ${ }^{2}$, air, air ${ }^{2}$, water, year) 
The primary purpose of basking in emydid turtles is for thermoregulation (Ben-Ezra and others, 2008), hence we included air and water temperatures as these conditions were likely to influence basking behavior. Wind and sky conditions also seemed likely to influence basking behavior, but including them did not improve the model based on Akaike Information Criterion (AIC). We included the covariate "year" to assess the trend in occupancy over years and the possibility that $p$ might vary over years with changes in habitat or field personnel. We also included "date" because changes in behavior and abundance over the season might affect $p$. Including the quadratic effects of date and air temperature on $p$ convincingly improved the model $(\triangle \mathrm{AIC}=6.4)$.

We included "bask" as a covariate of $\psi$ because the relative amount of available basking structures (such as, logs, limbs, and emergent rocks) is likely important. Water temperatures increase with distance from the dam, hence we also included "distance". Reese and Welsh (1998b) found that bank structure and water velocity were important characteristics of western pond turtle habitat, hence we included them to explore the effect of these site characteristics on $\varepsilon$.

To estimate initial occupancy $\left(\psi_{1}\right), \gamma$, and $\varepsilon$, we fit a colext model with the following covariates of each parameter:

$\psi_{1}$ (bask, distance), $\varepsilon(),. \gamma(),$.$p (bank, bask, vel, distance, date, date { }^{2}$, air, air ${ }^{2}$, water, year)

To compare the effects of site characteristics on $\varepsilon$, we fit the following variations on the $\varepsilon$ portion of the model leaving the $\psi, \gamma$, and $p$ portions as shown in previous model:

$$
\varepsilon(\text { bank, bask, vel, distance), } \varepsilon \text { (bank), } \varepsilon(\text { bask }), \varepsilon(\text { vel }), \varepsilon(\text { distance }), \varepsilon(.),
$$

in addition to a null model, $\psi_{1}(),. \varepsilon(),. \gamma(),. p($.). Probabilities of occupancy in subsequent years are derived from the estimates of $\psi_{1}, \gamma$, and $\varepsilon$, and we used a nonparametric bootstrap to obtain the standard errors for $\psi_{2}$ and $\psi_{3}$ (appendix L).

We ran a separate analysis on the surveyed ponds. To estimate initial occupancy $\left(\psi_{1}\right), \gamma$, and $\varepsilon$, we fit a colext model with the following covariates of each parameter:

$$
\psi_{1}(\text { depth }), \varepsilon(.), \gamma(.), \mathrm{p} \text { (wind,year). }
$$

To compare the effects of pond site characteristics on $\varepsilon$, we fit the following variations on the $\varepsilon$ portion of the model leaving the $\psi, \gamma$, and $p$ portions as shown in previous model:

$$
\varepsilon(\text { veg, bank, bask, depth), } \varepsilon \text { (bank), } \varepsilon \text { (bask), } \varepsilon(\text { veg), } \varepsilon(\text { depth }), \varepsilon(.) \text {. }
$$

\section{Abundance}

\section{Western Pond Turtles}

To estimate trends in abundance in basking western pond turtles, we used the function "pcountOpen" from the package "unmarked" in R. This function is based on the models of Royle (2004) and Dail and Madsen (2011), and fits an N-mixture model that accounts for the number of animals counted during a field survey is less than the number of animals present and that the difference is variable and unobserved (Royle, 2004; Dail and Madsen, 2011). N-mixture models require repeated count data to assess the probability of detection, and are based in the assumption of a closed population within a season, relaxing that assumption to an open population between seasons. We found that the negative binomial error structure fit our data better than the Poisson or zero-inflated Poisson structures based on AIC. We used the "trend" parameterization of the model, which estimated the trend in abundance instead of survival, and compared the best model with the "no-trend" parameterization to assess evidence for a trend. In the trend model, the parameter $\gamma$ is used as the finite rate of increase, which is often referred to as $\lambda$ in the ecological literature and that is how we refer to it in section, 
Results. We used $\mathrm{K}=50$ as an estimate of maximum population size within a site. The abundance models took days of computer time to run, so we could not do a full model comparison. For this analysis, we simplified the covariates from the occupancy model and estimated the trend in abundance by fitting:

$$
\mathrm{N}(\text { bask }), \lambda(.), p\left(\text { day, } \text { day }^{2}, \text { Atemp, Atemp }^{2}\right. \text {, water, bask, year) }
$$

\section{Results}

\section{Foothill Yellow-legged Frogs}

A total of 23 perennial waterways feeding the primary study area of the main stem Trinity River were identified from the National Hydrography Dataset (U.S. Geological Survey, 2001) (fig. 1). Of those tributaries, 13 were surveyed for foothill yellow-legged frogs. A tributary was not surveyed if landowner access was not granted $(\mathrm{N}=2)$ or the tributary was dry $(\mathrm{N}=8)$. Foothill yellow-legged frog breeding was detected in 5 of the 13 surveyed tributaries in 2013: Indian Creek, Weaver Creek, Oregon Gulch, Canyon Creek, and North Fork Trinity River. Six of the 13 tributaries surveyed did not contain breeding habitat and were not surveyed in subsequent years. The remaining two tributaries, Brown's and Rush Creeks contained habitat and continued to be surveyed annually. In 2014, Reading Creek was added, for a total of eight tributaries. Of these eight, breeding was detected in all years in Indian, Weaver, Oregon, Canyon, and North Fork Creeks. Breeding was detected in 2014 and 2015 in Reading and Brown's Creeks. No breeding was detected in Rush Creek.

\section{Occupancy}

We found that including "date", "water", "wind", and "weather" together or individually as covariates for detection did not improve the models based on AIC. Subsequently, we only considered $p$ (year) in our modeling.

When modeling effects of site characteristics on $\varepsilon$, the "pctmc" model, $\varepsilon$ (pctmc), was the most parsimonious based on $\mathrm{AIC}_{\mathrm{c}}$ (table 1). The remaining models had less support with $\triangle \mathrm{AIC}_{\mathrm{c}}$ between 3.3 and 14.6, and the null model could be dismissed with $\Delta \mathrm{AIC}_{\mathrm{c}}=52.2$. The probability of detecting foothill yellow-legged frogs at a site when the species was present increased over the years (table 2).

After accounting for variation in the probability of detection, the overall estimate of $\psi$ decreased over the 3 years of the study (table 2). The probability that a site unoccupied for 1 year was colonized the next year was quite low, with a mean value of 0.020 , whereas the probability that a site occupied for 1 year became unoccupied (locally extinct) the next year was fairly high with a mean of 0.25 in 2014 and 0.26 in 2015 (table 2). Naive occupancy rates were 0.11 in 2013, 0.21 in 2014, and 0.13 in 2015.

When only the data from the five GRTS panels were used, the estimate of $\psi$ followed a similar pattern, and the mean estimates of local extinction were comparable (table 2). Naive occupancy rates for the five panels were 0.15 in 2013, 0.22 in 2014, and 0.07 in 2015. 


\section{Western Pond Turtles}

Total basking western pond turtles observed on the main stem Trinity River for each survey was 96, 119, and 104 in 2013; 85, 116, and 111 in 2014; and 92, 75, and 91 in 2015. Naive occupancy rates were $0.34,0.44$, and 0.47 in 2013,2014 , and 2015 , respectively. For the ponds, naive occupancy rates were $0.61,0.44$ and 0.44 over the same years.

Summarizing the habitat characteristic at sites where basking western pond turtles were observed, immature willow and willow/hardwood mix were the dominant vegetation at over 77 percent of the sightings (fig. 2). A cut bank with exposed tree roots was observed in conjunction with 85 percent of the sightings, 93 percent of sightings were at locations with few to moderate numbers of basking structures, and 98 percent of sightings were at water depths greater than $10 \mathrm{~cm}$ (fig. 2).

\section{Occupancy}

For the models of extinction probability, there was not strong support for any of the covariates of extinction. The constant model, $\varepsilon($.$) had the lowest \mathrm{AIC}_{\mathrm{c}}$, followed by the global model with $\Delta \mathrm{AIC}_{\mathrm{c}}=$ 5.45 (table 3). Only the null model could be dismissed with $\Delta \mathrm{AIC}_{\mathrm{c}}=39.28$. We report values using the constant model, $\varepsilon$ (constant).

The probability of detecting western pond turtles at a site when the species was present varied among years (table 2), and this variation was best explained by the date of surveys, air temperature, water temperature, and year.

The overall estimate of $\psi$ increased over the 3 years of the study (table 2). The mean probability that a site unoccupied 1 year was colonized the next year was 0.25 and the mean probability that a site occupied 1 year became unoccupied the next year was 0.08 (table 2).

When only the panel GRTS were considered, estimates of $p$ suggested less interannual variability (table 2 ). The mean probabilities of colonization and extinction were similar at 0.17 and 0.15 respectively, and estimates of $\psi_{\mathrm{t}}$ did not vary much over the 3 years (table 2 ).

For the ponds, the null model had the lowest $\mathrm{AIC}_{\mathrm{c}}$, and none of the other models had much support, with $\triangle \mathrm{AIC}_{\mathrm{c}}$ greater than or equal to 10.74 (table 4 ). Based on the null model, the mean probability of detection was high at $0.71(\mathrm{SE}=0.045 ; \mathrm{CI}=0.61-0.79)$ and estimates of $\psi_{\mathrm{t}}$ indicated that occupancy rates decreased over the 3 years (table 2 ).

\section{Abundance}

Mean weighted counts of basking western pond turtles, incorporating variation in habitat and survey covariates, at all GRTS panels were 1.08, 1.10, and 1.04 annually from 2013 to 2015. Using data from all GRTS panels, the open abundance model suggested an increase in the number of basking western pond turtles with an instantaneous growth rate of $\lambda=1.11(\mathrm{SE}=0.089 ; \mathrm{CI}=0.95-1.29)$. The trend model $\left(\Delta \mathrm{AIC}_{\mathrm{c}}=0.00\right)$ was favored slightly over the no trend model $\left(\Delta \mathrm{AIC}_{\mathrm{c}}=2.81\right)$. 


\section{Discussion}

Key objectives for the foothill yellow-legged frog and western pond turtle populations monitoring protocols on the Trinity River was to estimate site-level probability of occupancy, colonization, and extinction rates for both species, and an additional trend in abundance for western pond turtles. Although it is not possible to make conclusive statements regarding trends or colonization and extinction rates with only 3 years of data, the occupancy and abundance models we fit demonstrate the usefulness of these models to assess these rates, and these models provide a baseline for assessing the status of these two populations.

For foothill yellow-legged frogs, our results suggest a decline in probability of site occupancy over the 3 years of the study. However, 3 years is likely too short of a period to establish a trend, and these data are better considered as a baseline for future monitoring. We found a relatively high local extinction rate and low colonization rate $(0.25$ and 0.02 , respectively), which may be suggestive of "sink" populations (Peterman and others, 2014), potentially supported by "source" populations in the tributaries. The probability of local extinction decreased in the presence of off-channel habitats (side channels, inlets, and backwaters). Although the time series is not long enough for a convincing analysis, these results suggest the importance of off-channel habitat for establishing and maintaining foothill yellow-legged frog breeding sites.

Based on historical surveys for foothill yellow-legged frogs on the Trinity River (Lind and others, 1996; Wheeler and others, 2014), we anticipated low occupancy rates and were concerned about limiting our surveys to the panel GRTS sample sites. However, our results indicate comparable estimates of $\psi$ for all and panel GRTS sample sites $(0.31,0.22$, and 0.17 compared to $0.35,0.20$ and 0.12 for 2013, 2014, and 2015, respectively; table 2). Hence, we recommend limiting foothill yellowlegged frog surveys to the 5 panel GRTS sample sites downstream of Steel Bridge, containing a total of 79 sample sites.

Our results for abundance trends and probability of site occupancy for western pond turtles on the Trinity River suggested increases in both metrics over the 3-year study. In contrast to what we observed for foothill yellow-legged frogs, the colonization probability for western pond turtles was relatively high (0.25), whereas the probability of local extinction was low (0.09). This may suggest that the western pond turtles are expanding into previously unoccupied sites. The dynamics for the ponds adjacent to the river showed the reverse, with declining rates of occupancy over the course of the study, and a lower contrast in probabilities of colonization and local extinction $(0.23$ and 0.15 , respectively; table 2).

Although 3 years is not a long enough time series to draw conclusions, there likely is a link between western pond turtles using pond habitats adjacent to the river and those turtles that use river habitats. The last 3 water years for Trinity River have all been designated dry or critically dry (http://www.trrp.net/restore/flows/water-year-summaries) which means low-volume spring releases and high spring and summer water temperatures (Snover and others, 2015). One possible explanation for declining probabilities of site occupancy in ponds (together with increasing probabilities of site occupancy on the river) is that, with the warmer water temperatures, more western pond turtles are using river habitat. Continued monitoring and studies directed at this question would be needed to clarify this link. 


\section{Decision Support Tools}

The monitoring protocols and preliminary results presented here provide means of tracking trends in probability of site occupancy and abundance for foothill yellow-legged frogs and western pond turtles. With continued efforts, they also can provide information on how site characteristics related to habitat changes correlate with the probability of local extinction. This information is invaluable to understanding the status of the populations over the long-term, and can provide insights into how dam operation and restoration decisions might affect demographic processes. The next logical step in synthesizing this information is the development of decision support tools.

The decision-making process for dam management and restoration activities that enhances both fish and other wildlife populations is inherently complex with multiple sources of uncertainty associated with the ecological system and conflicting management objectives. Decision support tools provide a way to formalize these complexities into a framework that links management actions with anticipated outcomes, and identifies sources of uncertainty. The primary goal of TRRP dam management and restoration activities is the rehabilitation of the salmonid populations, and decision support tools are being developed for this goal. Although some of the management actions can benefit fish and herpetofaunal populations, such as enhancing gravel bars that provide nursery habitat for fry and breeding habitat for frogs, other actions, such as maintaining cooler water temperatures to reduce fish disease, inhibit growth and development of frogs and turtles (Wheeler and others, 2014; Ashton and others; 2015; Snover and others, 2015). Decision support tools can provide a way to incorporate riparian wildlife maintenance objectives while still prioritizing salmonids.

Conceptual models are useful for defining the links between management actions and biological or ecological processes. Our conceptual models for the links between management decisions and population dynamics for foothill yellow-legged frogs and western pond turtles outline what this process might look like for the Trinity River (figs. 3 and 4). Management decisions on annual hydrographs, restoration work, and gravel augmentation will each have effects (positive and negative) on different life stages of foothill yellow-legged frogs and western pond turtles, and the potential cumulative effects, within and between seasons, from these actions can be tested using demographic models.

Initial population models will inherently contain uncertainty in many of the parameters, however, not all of the uncertainties will need to be resolved to adequately predict population responses. Sensitivity analyses can help identify key parameters where reducing uncertainty will improve management decisions. These analyses will help identify studies that are needed to reduce key parameter uncertainty. As new information is collected, models are confronted with empirical data from the monitoring efforts, including changes in occupancy and abundance, to test their ability to project population responses to dam management and restoration decisions.

Furthermore, linking the development of decision support tools for foothill yellow-legged frogs and western pond turtles to those being developed for fish would provide the ability to assess trade-offs between management decisions that take into consideration the current status of each population. For example, if foothill yellow-legged frog and western pond turtle populations appear to be within some pre-determined level of acceptable risk, management decisions that favor fish would be followed. However, if risks to foothill yellow-legged frog or western pond turtle populations exceed acceptable levels, it would trigger management decisions that favor those populations over fish. 


\section{Summary}

Foothill yellow-legged frogs and western pond turtles are considered species of concern in California, and the U.S. Fish and Wildlife Service is currently conducting status assessments of both species to determine if they should be listed under the Endangered Species Act. We have developed and implemented monitoring strategies for the Trinity River and associated water bodies for these species. We assessed probabilities of site occupancy, colonization and extinction for both species, and demonstrated that overall abundance trends can be estimated for western pond turtles. With only 3 years of data, it is not possible to make any inferences regarding the status or trends of these populations, but our results suggest a short term decline for foothill yellow-legged frogs and a short term increase western pond turtles in site occupancy.

\section{Monitoring Protocols}

\section{Protocol Narrative}

In the development of this monitoring protocol, we follow the recommendations of Oakley and others (2003) for standardizing the format and content of long-term monitoring protocols. This section contains detailed information on the background, survey design and justifications, links to informing management decisions, and an overview of the key features for each step of carrying out the protocol, with the anticipation that infrequent revision will be required for this section. The detailed steps for each feature of the protocol is described in separate Standard Operating Procedures (SOPs), and these are referenced throughout the narrative. Each SOP contains a table for future revisions as new methods and techniques are developed, both for collecting data and for data management. Supporting information including datasheets, metadata, and R scripts for analyzing data are included as appendixes.

\section{Background and Objectives}

The goal of the Trinity River Restoration Program is to protect and restore wildlife populations along the river. For herpetological species, the foothill yellow-legged frog and western pond turtle have been identified as key populations that are likely negatively affected by past and current dam operations. These two species are listed as species of concern by the state of California (Jennings and Hayes, 1994). Their status is currently under review by the U.S. Fish and Wildlife Service; they are being considered for listing under the Endangered Species Act (U.S. Fish and Wildlife Service, 2015a, 2015b).

Studies comparing foothill yellow-legged frogs and western pond turtle populations on the main stem of the Trinity River with populations on the unregulated South Fork Trinity River (see section, Introduction, for details) have suggested differences in population size, occupancy, juvenile growth rates, and age to maturity between these rivers, which likely relate to the ecological effects of the impoundment of the Trinity River. (for example, Lind and others, 1996; Reese and Welsh, 1998a, 1998b; Wheeler and others, 2014; Ashton and others, 2015; Snover and others, 2015). Specifically, densities of foothill yellow-legged frog egg masses along the Trinity River are a fraction of what occurs on the adjacent South Fork Trinity River (Lind and others, 1996; Wheeler and others, 2014), and juvenile growth rates and sizes of adult western pond turtles are markedly lower and smaller, respectively, on the main stem compared to those on the South Fork Trinity River (Ashton and others, 2015; Snover and others, 2015). 
The current need of the TRRP is a decision support tool that can incorporate the links between dam management decisions and population effects to anticipate how populations are likely to respond to management decisions, and what actions might be taken to enhance the viability of these populations. A key part of this larger project was an ongoing monitoring strategy that will track the status of these populations in terms of occupancy and abundance, changes in distribution, and habitat characteristics that are linked to changes in distribution. We developed the following monitoring protocols to collect data that can be analyzed with occupancy models (MacKenzie and others, 2003; Royle, 2004; Dail and Madsen, 2011). Our objective with this monitoring protocol was to provide TRRP with long-term monitoring protocols for foothill yellow-legged frogs and western pond turtles designed to: (1) estimate site-level probability of occupancy, colonization, and extinction rates for both species; (2) examine how site characteristics and habitat changes, especially as they relate to dam operation and restoration decisions, correlate with the probability of local extinction; and (3) estimate the overall trend in abundance for western pond turtles.

\section{Specific Monitoring Questions}

1. How do the foothill yellow-legged frog and western pond turtle populations respond to variable hydrographs?

2. Do the habitat modification efforts of TRRP alter the occupancy of foothill yellow-legged frog or western pond turtles?

3. Can management activities influence the trends in occupancy of foothill yellow-legged frogs or western pond turtles?

The proposed work supports strategic goals identified as priorities in the TRRP Integrated Assessment Plan and Conceptual Models and Hypotheses for the TRRP. Data collected under this protocol will assist TRRP in achieving specific management goals of determining if the proportion of habitats occupied by and(or) abundance of foothill yellow-legged frogs and western pond turtles is:

1. increasing or decreasing over time,

2. related to the water year and spring flows from the dam, and

3. related to specific habitat variables that can be modified through restoration activities.

\section{Sampling Design}

A GRTS with a rotating panel revisit design was developed for fish habitat monitoring work by TRRP partners (California Department of Fish and Game and others, 2010). Each GRTS sample site consists of a 400-m segment of the river, with segments defined based on the $5,000 \mathrm{ft}^{3} / \mathrm{s}$ centerline of the river bed. Fifty percent of the sampling universe is represented in 5 panels, each panel consisting of 16 GRTS sampling units. In the rotating panel design, 2 panels are surveyed each year, 1 of the panels from a previous year is included in the following year, and it takes 5 years to visit all panels. To enhance opportunities for integrating our monitoring data with that of the fish studies and other disciplines, we designate sample sites consistent with the GRTS sampling units; however, given the small population sizes and sparse distribution of our focal species, we did not adopt the rotating panel design. 


\section{Spatial Design}

\section{Foothill Yellow-legged Frogs}

For the foothill yellow-legged frogs, we visit the GRTS units from all five panels each year (fig. 5). Our initial intent for the frog surveys was to designate gravel bars within GRTS units as study sites. Hence, for the first 3 years, all GRTS sample units between Steel Bridge and the North Fork Trinity River were surveyed (100 percent of the sample universe). However, gravel bar habitats are dynamic and can be altered considerably between seasons depending on winter storm events, peak volume of the spring dam release, and ongoing rehabilitation work. In 2015, approximately 25 percent of previously designated gravel bar sample sites had either disappeared or moved a considerable distance. Hence we needed a study design that can account for, and characterize, changing foothill yellow-legged frogs breeding habitat. As a result, we consider each panel GRTS sample unit a sample site, for a total of 80 sample sites. Each sample site is assessed for the presence of breeding habitat, and if present, the habitat is surveyed and the amount of habitat is quantified. The amount of breeding habitat within each GRTS sample unit is then treated as a yearly site covariate in the modeling.

The TRRP is interested in understanding the metapopulation dynamics between the tributaries and the Trinity River. Specifically, do tributaries act as a source population for foothill yellow-legged frogs while the river acts as a sink? Although more focused studies are needed to truly address this issue, we include 400-m surveys, beginning at the confluence with the Trinity River, for those tributaries within the main study area that were determined to have breeding habitat (fig.5, SOP 3: Survey Techniques).

\section{Western Pond Turtles}

Western pond turtles are sparsely distributed along the Trinity River over the length of our study area (Lewiston Dam to the confluence with the North Fork Trinity River; fig. 6). Because of the expectation of numerous uninhabited areas (disproportionate numbers of zeros in the abundance counts), we surveyed the full length of the river study area (fig. 6), considering each 400-m GRTS sample unit to be a site, for a total of 156 sample sites.

The TRRP is interested in pond use by western pond turtles, hence we include ponds within 500 $\mathrm{m}$ of the centerline of the river within the study area. Thirty-five ponds were initially identified within $500 \mathrm{~m}$ of the center line of the main stem Trinity River primary study site from either previous studies (Sloan, 2012) or from the National Hydrography Dataset (http://www.nhd.usgs.gov). Two additional ponds were identified in the field. Of the 37 ponds, 20 were surveyed for western pond turtles (fig. 6). The remaining ponds were not included in the survey because initial site visits in 2013 indicated that they were dry $(\mathrm{N}=11)$ or could not be located $(\mathrm{N}=2)$, or we were denied access by landowners $(\mathrm{N}=4)$. 


\section{Frequency and Timing of Sampling}

\section{Foothill Yellow-legged Frogs}

Because of the low level of breeding detected in occupied sites on the Trinity River (typically one to two egg masses), sites transition from being truly unoccupied to occupied within a short time. In the early part of the season, initial surveys may predate the first oviposition event for a site, whereas later in the season, as larvae become mobile, they move into downstream habitats that were previously unoccupied. A key assumption of the occupancy and abundance models used to analyze these data is that there is no change in occupancy status between surveys within a season. Because of the amount of time required for each survey, we found that only two surveys could reasonably be accomplished while meeting this assumption, with a start date after oviposition had likely begun and a second survey completed prior to larvae moving to downstream habitats. Given the relatively high detection rates, less than 80 percent in 2014 and 2015, two surveys should provide reasonable estimates and variance from occupancy models.

Wheeler and others (2014) assessed the start and duration of foothill yellow-legged frog breeding on the Trinity River in studies between 2006 and 2009. We overlaid their results with water flow and water temperatures to assess when foothill yellow-legged frogs breeding surveys should commence (fig. 7). The start of surveys is dependent on the water year type (dry, normal, or wet). Breeding typically begins in late May and extends through mid-June, therefore we recommend initiating surveys in early to mid-June, once flows are less than $1000 \mathrm{ft}^{3} / \mathrm{s}$. However in wet years, such as 2006 and 2008 (fig. 7), breeding can be delayed, and surveys should be initiated later in June and at high flow rates. Breeding may coincide with a bench in the hydrograph, where flow rates are constant. The hydrographs are developed and published by mid to late April (http://www.trrp.net/), so the timing of surveys should be determined annually to correspond with features of the hydrograph, such as benches, flow rates, and predicted water temperature. The 3 years of our preliminary work developing these protocols were dry or critically dry years, so we were not able to investigate the timing of surveys in other water years. More work will be required to better describe the timing of monitoring under more varied conditions. Information is and should continue to be collected on the Gosner stage (Gosner, 1960) of egg masses to assist in establishing the timing of the onset of breeding under different wateryear conditions.

For the tributaries, four (Indian Creek, Weaver Creek, Reading Creek, and Oregon Gulch) contain warm waters as they do not originate in the Trinity Alps, and breeding is initiated considerably earlier than on the Trinity River or the other tributaries. Hence, surveys of these tributaries should take place between mid-April and early May. Surveys of the remaining tributaries should occur in late May to early June, preceding the main stem Trinity River surveys.

While surveying for foothill yellow-legged frogs, it was not uncommon to come across western pond turtles in shallow waters. As there have been extensive mark and recapture efforts on this population (Reese and Welsh, 1998a; Ashton and others, 2015; Snover and others, 2015), it was decided worthwhile to hand capture these western pond turtles, assess them for marks, and record basic morphometrics (SOP 3: Survey Techniques). At a minimum, these actions can provide additional growth and size-at-age data for this population. 


\section{Western Pond Turtles}

Western pond turtles use river habitats between approximately April and September, although there is some evidence that turtles may begin to move away from the river to terrestrial overwintering habitats as early as August within the study site (D. Ashton, written comm., 2013). Adult female western pond turtles typically nest from late May to mid-July based on records of gravid females (Ashton and others, 2010). Hence, we conducted surveys for basking western pond turtles starting in the second week of July, after the majority of adult females had ceased nesting, making them available for detection, and before turtles began upland migrations for overwintering. Three surveys were conducted per season.

\section{Field Methods and Rationale}

\section{Field Season Preparations and Equipment Setup}

Preparations for field work include decisions regarding the timing of the frog surveys based on the TRRP hydrograph (SOP 1: Field Preparation and Equipment Set-up). Equipment setup includes a test float of the kayaks, ensuring all electronic equipment is in working order, and inventorying all gear (SOP 1: Field Preparation and Equipment Set-up).

\section{Sequence of Events During the Field Season}

The TRRP will define the water year and publish the hydrograph by mid-to-late April of each year. This information can be accessed at http://www.trrp.net. Once the hydrograph is published, decisions will be made for the timing of the main stem Trinity River frog surveys. See appendix A for an example field schedule based on the 2015 water year. Generally, tributaries will be surveyed for foothill yellow-legged frogs for 2 days in late April, and for 1 week at the end of May to early June. Foothill yellow-legged frog surveys on the Trinity River occur in June and western pond turtle surveys on the Trinity River and associated ponds occur in July. Approximately 10 days and a 2-person staff (20 person-days) are required for post-processing data and end-of-season wrap-up (SOP 3: Survey Techniques, SOP 4: Data Management, and SOP 5: Spatial Data Management).

\section{Details of Making Observations}

\section{Foothill Yellow-Legged Frogs}

The general method for accessing sites and detecting foothill yellow-legged frogs, egg masses, and larvae using a two-person crew is consistent with the methods used previously on the Trinity River (Wheeler and others, 2014). The details for making these observations are covered in SOP 3: Survey Techniques. Briefly, a two-person crew floats the river, consulting the GPS for the start and end locations of the GRTS sample units. When habitat is identified, as defined in "Sampling Design", kayaks are secured to shore and surveys are conducted on foot, whereby one person walks the shoreline slightly ahead of the second person walking below the water line. Locations of foothill yellow-legged frog egg masses, larvae, juveniles, and adults are 
recorded, as well as the presence of any other herpetological species. If a foothill yellow-legged frog is detected and easily hand-captured, it should be captured, checked for identifying marks, photographed, and measured (appendix F). Only viable breeding habitat is surveyed, and separate tracks are recorded using a GPS to quantify the amount of habitat contained in the 400-m transects (SOP 3: Survey Techniques).

\section{Western Pond Turtles}

Our goal was to assess turtle status for the entire Trinity River study area. We developed a monitoring protocol to detect basking western pond turtles (Bury and others, 2012). Western pond turtles on the Trinity River spend substantial periods of time basking out of the water, using primarily woody vegetation and rocks as substrates (Reese and Welsh, 1998a; Ashton and others, 2010). We used analytical methods that compensate for the effect of water temperature and other factors on the number of western pond turtles observed, so that estimates of the probability of site occupancy and trend in abundance are not biased.

Details of conducting surveys for basking western pond turtles are covered in SOP 3: Survey Techniques. Briefly, a two-person crew in separate kayaks floats the primary study area, proceeding as slowly as possible. Each crew member is assigned a river bank (river right or river left) and the banks are scanned downriver using binoculars. All basking western pond turtles are counted prior to approaching the site, as turtles will drop into the river as the kayaks approach. Once the observer is at the location of where the western pond turtles were sighted, a waypoint is marked on the GPS, and the waypoint number and habitat characteristics are recorded (SOP 3: Survey Techniques). When deemed safe, all side channels and backwaters immediately adjacent to the main channel also are surveyed. Surveys of the primary study area are repeated three times (SOP 3: Survey Techniques).

\section{Post-Collection Datasheet Processing}

At the end of the day, after returning to the crew quarters, datasheets are reviewed for completeness, notes are entered as needed to clarify any observations (SOP 3: Survey Techniques).

\section{End-of-Season Procedures}

At the end of the season, all datasheets are scanned to TIFF file format and stored in an appropriate network folder (SOP 3: Survey Techniques). All data are entered into the Access ${ }^{\circledR}$ database and checked for accuracy (SOP 4: Data Management).

All gear is cleaned and thoroughly dried then stored (appendix B, SOP 3: Survey Techniques). 


\section{Data Handling, Analysis and Reporting}

\section{Data Management}

We created a relational database in Microsoft ${ }^{\circledR}$ Access for the frog and turtle data. The database will be maintained by USGS on ScienceBase (https://www.sciencebase.gov) when data are collected by USGS. A copy of the database also will be maintained by TRRP, with updates provided annually. Data are collected on field datasheets (appendixes F and G), and these data are entered into the database by technicians familiar with the data collection protocols. Data verification will be conducted within 1 month of data entry. Scans of the datasheets and the originals will be archived at USGS, Forest and Rangeland Ecosystem Science Center (SOP 3: Survey Techniques).

Analysis and Reporting

Analysis

Data summaries presenting the percentage of sites occupied by foothill yellow-legged frogs and western pond turtles, as well as species detection probabilities, will be prepared annually. In addition, abundance estimates will be prepared for western pond turtle. Preliminary statistical analysis on the first 3 years of data have been completed and are included with this report, and they will be updated at least every 5 years (SOP 6: Data Analysis and Reporting, appendixes J-N).

\section{Reporting}

Annual reports based on data of the current year, including data summaries, will be prepared. The report, database, and metadata will be transmitted to TRRP by December 31 (SOP 6: Data Analysis and Reporting).

\section{Personnel Requirements and Training}

The monitoring requires two technicians, including one lead with at least one season of experience conducting similar surveys or monitoring. The lead technician will provide training in kayak/boating safety and the monitoring protocols as needed to the second technician (SOP 2: Personnel and Training).

The data management and analysis requires a technician with a quantitative background, experience manipulating large datasets, and experience using occupancy models in R. 


\section{Operational Requirements}

\section{Annual Workload and Field Schedule}

A 2-person crew is required. Foothill yellow-legged frog surveys of the warmer tributaries will be conducted in late April, with 2 surveys of each completed in 2 field days. The colder tributary surveys will be completed in late May through early June. Timing of the main stem surveys will be determined based on the hydrograph. A total of about 16 field days are required to complete the frog surveys. Turtle surveys will be completed in about 12 field days during mid to late July.

\section{Facility and Equipment Needs}

This work requires two inflatable kayaks and two vehicles to manage shuttles between put-in and take-out locations (only one vehicle is required for the early tributary surveys). SOP 1: Field Preparation and Equipment Set-up contains a list of specific field gear required. Normal office space and computing abilities are needed for the data entry work. The TRRP office in Weaverville, California, provides field crews with needed office equipment such as printers, scanners, and internet access while conducting field work.

\section{Protocol Revision}

Revisions to this protocol may be necessary for several reasons, including improvements in survey techniques, new modeling, statistical approaches, or new objectives. Documentation of these changes is needed to ensure consistency in data collection and analysis. This document has been divided into the protocol narrative and supporting SOPs such that minor changes will not require a revision of the entire protocol. The SOPs contain detailed information on specific tasks required to complete the monitoring, and are more likely to require revision than the protocol narrative. All versions of the protocol narrative and SOPs shall be archived, and a history log shall be filed. Detailed steps on completing revisions of this protocol are in SOP 7: Revising the Protocol. 


\section{Standard Operation Procedures for Monitoring Herpetological Species}

Standard Operating Procedure (SOP) 1: Field Preparation and Equipment Set-up

\begin{tabular}{c|c|c|c|c|c}
\hline $\begin{array}{c}\text { Previous } \\
\text { Version No. }\end{array}$ & $\begin{array}{c}\text { Revision } \\
\text { Date }\end{array}$ & Author & Changes Made & Reasons for Change & $\begin{array}{c}\text { New Version } \\
\text { No. }\end{array}$ \\
\hline 1.0 & & & & & \\
\hline & & & & & \\
\hline & & & & & \\
\hline
\end{tabular}

1. Purpose.

1.1. The purpose of this Standard Operating Procedure (SOP) is to prepare field crews for herpetological monitoring along the Trinity River, including developing the schedule, becoming familiar with relevant species, and assembling the field gear.

2. Reference Documents.

- Gosner, K.L., 1960, A simplified table for staging anuran embryos and larvae with notes on identification: Herpetologica, v. 16, p. 183-190.

- Stebbins, R.C., 2003, A field guide to western reptiles and amphibians: Houghton-Mifflin, New York, $533 \mathrm{p}$.

- Appendix B. U. S. Geological Survey Hygiene Protocol for Control of Disease Transmission between Amphibian Study Sites .

- Wheeler, C.A., Bettaso, J.B., Ashton, D.T., and Welsh, H.H. Jr., 2014, Effects of water temperature on breeding phenology, growth and metamorphosis of foothill-yellow-legged frogs (Rana boylii) - a case study of the regulated main stem and unregulated tributaries of California's Trinity River: River Research and Applications, DOI: 10.1002/rra.2820.

3. Procedures and General Requirements

3.1. The Trinity River Restoration Program (TRRP) will define the water year and publish the hydrograph by mid- to late April of each year. This information can be accessed at http://www.trrp.net. Once the hydrograph is published, the project lead will decide on the timing of the main stem Trinity River foothill yellow-legged frog surveys. This will take into consideration the descending limb of the hydrograph, location of benches, and projected flows and temperature. A tentative schedule is then prepared for the field season (appendix A). Staff should be hired to start at least 1 week prior to the start of the main field season. General preparation:

3.1.1. At least 6 months prior to the field season, ensure that the lead technician's California Department of Fish and Wildlife Scientific Collecting Permit is current, renew as necessary.

3.1.2. At least 3-4 months prior to the field season, ensure that the Animal Care and Use Proposal (ACUP) is current and make any amendments as necessary.

3.1.3. Review the protocol narrative and all SOPs.

3.1.4. Ensure familiarity with species identification for those likely to be encountered (see datasheet, appendix F).

3.1.5. Maps identifying generalized linear random tessellation stratified (GRTS) sample unit locations, 400-m tributary transects, and pond locations should be loaded onto the handheld GPS or be available on a laptop computer to be taken into the field.

3.1.6. Update western pond turtle habitat datasheets with the habitat classifications of previous year.

3.1.7. Assemble, clean and disinfect, and repair all field equipment as needed. 


\subsection{Scheduling Field Work}

3.2.1. The field schedule will begin in late April with a 2-day site visit (not including travel time) to survey the warm water tributaries for foothill yellow-legged frogs. The remaining tributaries will be surveyed the week after the Memorial Day holiday (the last Monday in May). The timing for the start of the main stem frog surveys will be based on the hydrograph. Surveys for western pond turtles will begin the second week of July, and all field work should be completed by the end of July.

3.2.2. For dry and critically dry years, main stem frog surveys should be initiated in approximately the second week of June to ensure breeding has commenced. As mentioned previously, additional work will be required to pinpoint the timing for other water years. Key features of the hydrograph, such as flow and benches, together with the historical work of Wheeler and others (2014) should be consulted to determine when foothill yellowlegged frog surveys should be initiated.

3.2.3. A crew of 2 people is required for all field work. There will be 2 field days in late April. The remaining foothill yellow-legged frog surveys will require 14 field days and the western pond turtle surveys will require 12 field days.

3.3. Prior to leaving the lab for a site visit, the following tasks should be completed:

- Make copy of current Scientific Collecting Permit from California Department of Fish and Wildlife (previously California Department of Fish and Game).

- Contact Michele Gallagher (magallagher@usbr.gov) or the current public outreach specialist at TRRP to inform of the general dates you will be working and what general activities you will be doing.

- Review itinerary, maps, and check-in procedure.

- Contact relevant agency personnel, private landowners, and other relevant parties to discuss access issues prior to surveys. Crews should contact the laboratory with any questions.

- Inform the California Department of Fish and Wildlife of the intent to collect specimens, as per scientific collecting permit requirements.

- Perform vehicle safety checks before loading gear (complete the checklist in vehicle notebook).

- Carry adequate maps to navigate isolated roads.

- Review hard copy forms to ensure they reflect any changes or updates in database. Make copies of blank map sheets and backup datasheets (appendixes F and G).

- Leave a detailed itinerary with supervisors and coworkers at office before leaving.

- Designate a point-of-contact who will be kept apprised of daily activities.

- Ensure that the tracks and waypoints from previous year have been removed from the GPS.

\subsection{Field Equipment}

3.4.1. All field equipment should be assembled, cleaned, and checked for operability at least 2 weeks prior to the anticipated start of the field season, or when the equipment will be required (kayaks are not needed until the start of the main field season). Faulty equipment should be repaired or replaced. Kayaks should be inflated and taken for a short float on a local water body to ensure there are no leaks.

3.4.2. A hygiene kit should be prepared with the supplies required to follow the hygiene protocol (appendix B). 
3.5. List of Field Equipment for the Two-Person Survey Crew:

- Itinerary with contact information

- Collecting permit

- Protocol for survey

- Identification guides/keys

- Kayaks

- Repair kit

- Paddles

- Personal flotation devices with whistle, light source, and signaling device attached

- Air pump (two, large for vehicles and small to take on water)

- Dry bags

- Rope/straps/carabineers (strap bag)

- Polarized sunglasses

- Binoculars

- Datasheets and clipboard

- Hygiene materials:

- Protocol

- Bleach

- Measuring container

- Scrub brush

- Bucket

- Spray bottle

- GPS and spare AA batteries (about 2 spares per week of field work)

- Maps

- Cell phone and car charge cable

- SPOT $^{\circledR}$ device (personal GPS tracker)

- Pencils, extra lead, Sharpie ${ }^{\circledR}$ marker

- Water shoes

- Thermometers for air and water temperatures

- Rulers (2)

- Tape measure

- Water resistant watch with stop watch function

- Camera and spare battery

- Emergency gear including vehicle emergency preparedness pack and small first aid kits for boats

- Extra water

- Appropriate clothing for cold/wet weather and spare clothing

- Calipers

- Scales

- Sharpie ${ }^{\circledR}$ pen 
Standard Operating Procedure (SOP) 2: Personnel and Training

Revision History Log:

\begin{tabular}{c|c|c|c|c|c}
\hline $\begin{array}{c}\text { Previous } \\
\text { Version No. }\end{array}$ & $\begin{array}{c}\text { Revision } \\
\text { Date }\end{array}$ & Author & Changes Made & Reasons for Change & $\begin{array}{c}\text { New Version } \\
\text { No. }\end{array}$ \\
\hline 1.0 & & & & & \\
\hline & & & & & \\
\hline & & & & & \\
\hline
\end{tabular}

1. Purpose

It is critical that species are correctly identified. The statistical procedures used to analyze these data do not allow for incorrect (false positive) identifications. This protocol also involves kayaking, and it is important that staff receive appropriate safety training. The purpose of this document is to help ensure that field crews are properly trained.

2. Reference Documents

- Stebbins, R.C., 2003, A field guide to western reptiles and amphibians: Houghton-Mifflin, New York, $533 \mathrm{p}$.

3. Procedures and General Requirements

3.1. Personnel requirement

3.1.1. The team lead should have at least one season of experience working on the Trinity River Herpetological Monitoring Project or other similar project.

3.1.2. The second crew member should have a minimum of a Bachelor of Science degree in Biology, Wildlife Biology, Environmental Science, or a related degree. Previous experience with field work, especially with amphibians, is desirable but not necessary.

3.2. Identification of key species

3.2.1. Western pond turtles are the only turtle species likely to be observed, hence identification is straightforward.

3.2.2. Foothill yellow-legged frog egg masses are distinct from other anuran egg masses in the area and are readily identified. The only other species of juvenile and adult anurans likely to be observed are Northern Pacific tree frogs (Pseudacris regilla; PSRE), American bullfrogs (Lithobates catesbeianus; LICA) and Western toads (Anaxyrus boreas; ANBO), which are readily distinguished from foothill yellow-legged frogs.

3.2.3. Excluding bullfrogs, larvae from the other two species often occur within breeding habitat, and differentiating between them is critical. At a minimum, the lead crew member should have some expertise in distinguishing between the three species and provide training to the second crew member as needed. 


\subsection{Equipment}

3.3.1. New crew should receive training in the use of the global position system (GPS), including loading maps, downloading waypoint and tracks, recording waypoints, and recording and naming tracks.

3.3.2. New crew should receive training in kayaking and kayak safety. Throughout the study site, the Trinity River is relatively calm and at low water there is nothing larger than a Class 1 rapid. However, if a crew member does not have experience in river kayaking, time should be taken at the beginning of the season, in conjunction with the late May tributary surveys, to instruct crew on paddling techniques, steering kayaks, avoiding hazards, safely entering and navigating rapids and riffles, eddying out, and securing kayaks. A float between the Rush Creek River Access and the Bucktail River Access is a good stretch of easy river for this instruction. The first survey of Rush Creek can be conducted at the same time (appendix A). At high water levels, such as was seen in 2006, the river is likely to present more dangers and care should be taken on selecting experienced crew and timing the start of surveys.

\subsection{Additional Training}

3.4.1. Ensure that all field staff are current on First Aid/CPR certification.

3.4.2. Ensure enough time for field staff to complete all center-required safety training prior to the start of field work.

3.4.3. Ensure all field staff complete the Animal Care and Use Protocol (ACUP) training requirements. 
Standard Operating Procedure (SOP) 3: Survey Techniques

Revision History Log:

\begin{tabular}{c|c|c|c|c|c}
\hline $\begin{array}{c}\text { Previous } \\
\text { Version No. }\end{array}$ & $\begin{array}{c}\text { Revision } \\
\text { Date }\end{array}$ & Author & Changes Made & Reasons for Change & $\begin{array}{c}\text { New Version } \\
\text { No. }\end{array}$ \\
\hline 1.0 & & & & & \\
\hline & & & & & \\
\hline & & & & & \\
\hline
\end{tabular}

1. Purpose

The purpose of this document is to outline the procedures for conducting surveys for foothill yellowlegged frogs and western pond turtles on the Trinity River and associated water bodies.

2. Reference Documents

- Bury, R.B., Welsh, H.W. Jr., Germano, D.J., and Ashton, D.T. eds., 2012, Western pond turtlebiology, sampling techniques, inventory and monitoring, conservation, and management: Northwest Fauna Number 7, Society for Northwestern Vertebrate Biology, Olympia, 128 p.

- $\quad$ Reese, D.A., and Welsh, H.H. Jr., 1998a, Comparative demography of Clemmys marmorata populations in the Trinity River of California in the context of dam-induced alterations: Journal of Herpetology, v. 32, p. 505-515.

- Stebbins, R.C., 2003, A field guide to western reptiles and amphibians: Houghton-Mifflin, New York, $533 \mathrm{p}$.

- Wheeler, C.A., Bettaso, J.B., Ashton, D.T., and Welsh, H.H. Jr., 2014, Effects of water temperature on breeding phenology, growth and metamorphosis of foothill-yellow-legged frogs (Rana boylii) - a case study of the regulated main stem and unregulated tributaries of California’s Trinity River: River Research and Applications, DOI: 10.1002/rra.2820.

3. Procedures

3.1. Each day, prior to starting surveys:

3.1.1. Inform the point-of-contact of your itinerary for the day, including put-in and take-out locations, and anticipated completion time.

3.1.2. Ensure all maps needed for the day have been loaded onto the global positioning system (GPS).

3.2. At the end of the day, inform the point-of-contact that you are finished and off-effort, either by cell phone or with the SPOT $^{\circledR}$ device if there is no cell service.

3.3. General procedures for field days that involve kayak floats:

3.3.1. Establish the put-in and take-out locations, this information should be communicated to the point-of-contact.

3.3.2. Drive both vehicles to the take-out location, ensuring all of the gear is in one of the vehicles.

3.3.3. Ensure both crew members can identify the location of the river take-out location . Walk to the river edge and note identifying features.

3.3.4. The vehicle without the gear is left at this location, and the crew proceeds in the other vehicle (with the gear) to the put-in location.

3.3.5. Unload gear as close to the river edge as possible. After all gear is unloaded, move the vehicle to a safe parking place.

3.3.6. Inflate the kayaks and stow and secure all gear.

3.3.7. At the end of the day, upon reaching the take-out location, beach the kayaks, drive the vehicle to as close as possible to the kayaks, partially deflate the kayaks, and load all gear. 
Drive to the put-in location and retrieve the other vehicle, then proceed to the crew quarters (motel, campground, or other lodging).

3.4. General survey procedures for foothill yellow-legged frogs on the Trinity River:

3.4.1. The GPS should be readily accessible and loaded with the generalized linear random tessellation stratified (GRTS) sample unit maps for the area being surveyed.

3.4.2. Begin surveying for breeding habitat as soon as kayaks are launched, or at the launch location, if applicable.

3.4.3. Record GRTS\# metrics on the RABO Surveys - Subhabitat Data datasheet (appendix F).

3.4.4. Viable habitat is defined by shallow edgewater, typically with gravel or cobble substrate. Egg masses will typically occur in areas with no canopy cover.

3.4.5. When viable habitat is detected, if it is safe, eddy out and secure kayaks to conduct a detailed survey.

3.4.6. If viable habitat is inaccessible for any reason, unsafe conditions or private property issues, make a note of the general location and indicate that detailed frog surveys could not be conducted.

3.4.7. Once at a habitat site, record River Side, Dominant Vegetation, Canopy Cover, Substrate, Water Temp (in the main channel), Air Temp, Wind, and Weather using the RABO Surveys - Subhabitat Data datasheet (appendixes C and F).

3.4.8. Assess and record the habitat types that are within the subhabitat unit; choose from main channel, backwater, inlet, or side channel (appendix C).

3.4.9. Initiate a new track on the GPS if this is the first visit to the site for the season. Create separate tracks for different habitat types, appending the track name with BW (backwater), IN (inlet), or SC (side channel). No acronyms will be used for Main Channel. Record track names on the datasheet.

3.4.10. Enter the start time, start a stopwatch, and begin surveying the site for egg masses, larvae, juveniles, and adults. Work together to survey shorelines, covering all of site that is physically accessible including gravel bars, islands, and side channels. Move slowly along the edge of the shoreline, scanning the water as well as the nearby shore/vegetation for egg masses that may have stranded as a result of declining water levels. Polarized glasses should be used consistently.

3.4.11. When egg masses are encountered, note the location, record the total number, and continue surveying. Once survey is complete, return to oviposition site and collect all data and enter on the RABO Monitoring Oviposition \& Eggmass Data datasheets (see section 3.4.16).

3.4.12. When adult or juvenile foothill yellow-legged frogs are encountered, record the Count, Stage, and SubHabitatID on theRABO Surveys - Detections datasheet (appendix F), then continue surveying.

3.4.13. When other herpetological species are encountered, record Species, Count, Stage, and SubHabitat ID and continue surveying (appendix F).

3.4.14. When incidental western pond turtles (ACMA) are encountered and can be readily hand captured, stop the stopwatch and complete the ACMA Incidental Capture Data datasheet (appendix F). When datasheet is complete, return the turtle to the capture location, resume stopwatch and continue the foothill yellow-legged frog survey.

3.4.14.1. Estimate Age, as described in Bury and others (2012).

3.4.14.2. Carapace measurements, are taken as described in Bury and others (2012). 
3.4.14.2.1. Maximum straight carapace length (Max SCL), measured with calipers from the outer edge of the first or second marginal scute to the last marginal on either side of the midline.

3.4.14.2.2. Minimum straight carapace length (Min SCL), measured with calipers from the nuchal notch to the cleft between the last marginal, along the midline of the carapace.

3.4.14.2.3. Straight carapace width (SCW), measured with calipers at the widest part of the carapace, perpendicular to the midline.

3.4.14.2.4. Depth is the shell height, measured with calipers from the plastron to the carapace at the deepest part of the body.

3.4.14.2.5. Maximum straight plastron length (Max SPL), measured with calipers flat against the plastron at the outer edges of the anterior- and posterior-most scutes.

3.4.14.2.6. Minimum straight plastron length (Min SPL), measure with calipers along the midline with the calipers inserted into the clefts between the anteriorand posterior-most scutes.

3.4.14.2.7. Straight plastron width (SPW), measured with calipers at the widest part of the plastron, perpendicular to the midline.

3.4.14.3. Note the location of any notches in the marginal scutes of the carapace that indicate the turtle was previously captured and marked. Record the Mark number or ensure photos and notes will enable determining the mark number.

3.4.14.4. Determine Sex and reproductive condition (Gravid) of adult females following Bury and others (2012).

3.4.14.5. If turtle is unmarked, use a Sharpie ${ }^{\circledR}$ pen to write a temporary ID (Temp Mark) on the caraspace ("subhabitat ID - turtle number", 1 for first capture, 2 for second, and so on) in case the turtle is recaptured on a subsequent survey

3.4.14.6. Take photographs with ruler flush against the carapace/plastron that clearly shows:

- the carapace and any marks on the marginal scutes,

- the full plastron, and

- a close up of posterior half of plastron in clear focus to confirm age.

3.4.15. After a survey has been finished, log the stop time, stop and name the track, and make any notes about the site.

3.4.16. Return to any egg mass locations and complete the RABO Monitoring Oviposition \& Eggmass Data datasheets (appendix F).

3.4.16.1. Record the water column depth at each cluster.

3.4.16.2. Record the substrate classification and waypoints for each cluster.

3.4.16.3. For each egg mass within each cluster, estimate the Gosner developmental stage (see chart in appendix I).

3.4.16.4. When estimating \% dead (the percentage of embryos in a given mass that appear dead), attempt to estimate this to the nearest 5 percent.

3.4.17. Review the habitat fields for completeness prior to leaving the site. 
3.5. General survey procedures for foothill yellow-legged frogs for tributaries.

3.5.1. Generally, the protocols for surveying the tributaries are the same as for the main stem Trinity River with a few exceptions.

3.5.2. Start at the confluence of the tributary and the main stem Trinity River. For wide tributaries, work up one side, following the same survey procedures as for main stem sites. Survey only those areas with potential habitat as described in paragraph 3.4 .4 within $400 \mathrm{~m}$ of the confluence.

3.5.3. Create separate tracks in the GPS for disconnected habitat to enable measuring total habitat.

3.5.4. For narrow channels where it is reasonable to survey both banks simultaneously, observers should walk below the waterline, scanning for egg masses in the water and foothill yellow-legged frogs along the shore.

3.6. General survey procedures for western pond turtles on the Trinity River:

3.6.1. The entire stretch of the study area, from the Lewiston Weir to the confluence with the North Fork, is surveyed.

3.6.2. The main stem is divided into 400-m segments based on the GRTS survey design used in the fish biology studies.

3.6.3. Identify the put-in and take-out locations for the day, and ensure the GRTS maps needed for the day are loaded on the GPS.

3.6.4. The first pass through all sites will involve characterizing the dominant habitat for each 400-m reach (see appendix D for habitat characteristics and appendix G for Datasheet 1). Compare the habitat with the previous year assessment to determine if the habitat has changed, otherwise keep the habitat assessment consistent with the previous year.

3.6.5. Once the habitat character survey has been completed, three surveys will be conducted for basking western pond turtles using Datasheet 2 (appendix G).

3.6.5.1. Assign a bank to each observer, alternating banks for different surveys. Begin surveying immediately after kayaks are launched. Float as slowly as possible, scanning downstream along your assigned bank with binoculars.

3.6.5.2. Record the time at each transition between GRTS unit segments.

3.6.5.3. Record the dominant Wind and Weather for each GRTS unit.

3.6.5.4. Have one observer record Water Temp and the other record Air Temp for each GRTS unit.

3.6.5.5. When a basking turtle is observed, note location and get an accurate Count of western pond turtles prior to approaching. Once you are perpendicular to the location, record a Waypoint (the turtle(s) likely will no longer be visible) and characterize the habitat (see appendix D).

3.6.5.6. If the main channel splits around an island and is deemed navigable on both sides, one observer should float each channel, observing both their river bank and the bank of the island for western pond turtles. If it is not deemed safe to float a side channel, one or both observers should get out at the island and observe the other bank. In addition to side channels, all backwaters should be observed for western pond turtles. 
3.7. General survey procedures for western pond turtles in ponds:

3.7.1. Appendix $\mathrm{H}$ lists the locations of the 20 pond sample sites.

3.7.2. Identify a vantage point that provides a view of as much of the pond as possible. Once at the vantage point, remain still for 5 minutes before beginning observations, do not observe the pond during this time. Following the quiet time, have one observer observe the pond for a reasonable period of time; $2-3$ minutes for small ponds; 5 minutes for medium ponds; and 9-10 minutes for large ponds. The western pond turtles will primarily be "waterbasking" with only their snouts showing above the water. The second observer should not be looking at the pond while the first observer is on effort. Once the first observer completes their observations, the second observer begins. For the third survey, the first observer does a repeat observation. Record all herpetological species seen or heard and record habitat characteristics (see Datasheet 3 and Datasheet 4 in appendix G).

3.8. General procedures for daily post field activities:

3.8.1. After returning to crew quarters, review all paper datasheets for completion. Add notes as necessary to clarify data entries.

3.8.2. If needed, disinfect gear and shoes (appendix B).

3.8.3. Download and label photographs taken that day.

3.8.4. Download all tracks and waypoints from the GPS, but do not clear them from the GPS.

3.8.5. Plan itinerary for following day and communicate that information to the point-ofcontact.

3.9. General procedures for end of season activities

3.9.1. Thoroughly clean the kayaks and ensure that they are completely dry prior to storage.

3.9.2. Inspect kayaks for leaks and repair as needed. The gear box contains instructions and materials for repairs.

3.9.3. Once dry, fully deflate the kayaks and roll them up for storage

3.9.4. Clean and dry the remaining, non-electronic gear and store in plastic tub with kayak (except for paddels that are stored with the kayaks).

3.9.5. For the electronic gear (GPS, camera, digital thermometers), inspect the battery housings for sand/dirt and clean as needed. Store these in a climate controlled space. 
Standard Operating Procedure (SOP) 4: Data Management

Revision History Log:

\begin{tabular}{c|c|c|c|c|c}
\hline $\begin{array}{c}\text { Previous } \\
\text { Version No. }\end{array}$ & $\begin{array}{c}\text { Revision } \\
\text { Date }\end{array}$ & Author & Changes Made & Reasons for Change & $\begin{array}{c}\text { New Version } \\
\text { No. }\end{array}$ \\
\hline 1.0 & & & & & \\
\hline & & & & & \\
\hline & & & & & \\
\hline
\end{tabular}

1. Purpose

The purpose of this document is to detail the steps that should be taken to manage the data after it has been collected, including data entry, quality assurance/quality control checks, and curating.

2. Reference Documents

- None

3. Procedures

3.1. Make digital scans of all datasheets and maps in TIFF file format to an appropriate shared network drive.

3.2. Create a blank copy of the Access ${ }^{\circledR}$ database and save it as "Trinity YEAR.accdb", where YEAR is the year of the current field season.

3.3. This is a relational database and data must be entered following the hierarchical structure. For example, an entry for Survey 1 must be created on the Survey sheet prior to entering information on the subhabitat sheet.

3.4. Ideally, staff involved in the collection of the data will enter data into the database.

3.5. When all data are entered, they should be checked for quality assurance/quality control by comparing all entered data to that recorded on the datasheets. Data should be edited as necessary to correspond with those recorded on the datasheets.

3.6. Data for track lengths and waypoint locations are generated from SOP 5: Spatial Data Management and should be recorded and checked for quality assurance/quality control.

3.7. When the database is complete, it should be merged with the Trinity All.accdb, which contains data from all years of surveys.

3.8. File original datasheets, provide copies to the USGS Forest and Rangeland Ecosystem Science Center data manager.

3.9. This updated database is then transmitted to the Trinity River Restoration Program (TRRP) through email and transmitted to the USGS Forest and Rangeland Ecosystem Science Center Data Manager to be curated on ScienceBase (https://www.sciencebase.gov). The database also is publically available through the TRRP data portal (http://odp.trrp.net). 
Standard Operating Procedure (SOP) 5: Spatial Data Management

Revision History Log:

\begin{tabular}{c|c|c|c|c|c}
\hline $\begin{array}{c}\text { Previous } \\
\text { Version No. }\end{array}$ & $\begin{array}{c}\text { Revision } \\
\text { Date }\end{array}$ & Author & Changes Made & Reasons for Change & $\begin{array}{c}\text { New Version } \\
\text { No. }\end{array}$ \\
\hline 1.0 & & & & & \\
\hline & & & & & \\
\hline & & & & & \\
\hline
\end{tabular}

1. Purpose

1.1. The purpose of this document is to detail the steps to manage and analyze the data collected by the handheld global positioning system (GPS) that includes tracks for the foothill yellow-legged frog breeding habitat and waypoints recording the locations of egg masses, foothill yellow legged-frogs and basking western pond turtles.

2. Reference Documents

- None

3. Procedures for foothill yellow-legged frog habitat tracks.

3.1. Connect the GPS to a computer and open the DNRGPS application (accessed at http://www.dnr.state.mn.us/mis/gis/DNRGPS/DNRGPS.html).

3.2. Under the Track tab, select Download, this should show all tracks recorded on the GPS.

3.3. Under the File tab, select Save To, then ArcMap, and save the file as a polyline shapefile labeled RABOTracks YEAR, where YEAR is the current survey year.

3.4. Open the shapefile in ArcMap using the Universal Transverse Mercator (UTM) coordinate system and navigate to the attribute table for the shape file.

3.5. Open an editing session and add length, in meters, to each track.

3.6. Review the tracks to ensure reasonable start and end locations, and that the tracks are relatively free of excessive wandering. Any tracks that seem suspect should be hand measured using the ArcMap measuring tool.

3.7. Export the attribute table to excel and record the track lengths in the database.

4. Procedures for Waypoints:

4.1. Connect the GPS to a computer and open the DNRGPS application (accessed at http://www.dnr.state.mn.us/mis/gis/DNRGPS/DNRGPS.html).

4.2. Under the Waypoint tab, select Download. This should show all waypoints recorded on the GPS.

4.3. Under the File tab, select Save To, then ArcMap, and save the file as a point shapefile labeled TrinityWaypointsYEAR, where YEAR is the current survey year.

4.4. Open the shapefile in ArcMap and navigate to the attribute table for the shape file.

4.5. Review the waypoint locations in reference to the river.

4.6. Export the shapefile to Excel $^{\circledR}$.

4.7. For each waypoint number recorded in the database, enter the latitude and longitude from the exported shapefile to the appropriate fields in the database. 
Standard Operating Procedure (SOP) 6: Data Analysis and Reporting

Revision History Log:

\begin{tabular}{c|c|c|c|c|c}
\hline $\begin{array}{c}\text { Previous } \\
\text { Version No. }\end{array}$ & $\begin{array}{c}\text { Revision } \\
\text { Date }\end{array}$ & Author & Changes Made & Reasons for Change & $\begin{array}{c}\text { New Version } \\
\text { No. }\end{array}$ \\
\hline 1.0 & & & & & \\
\hline & & & & & \\
\hline & & & & & \\
\hline
\end{tabular}

1. Purpose

1.1. The purpose of this document is to detail the steps involved in analyzing the data.

2. Reference Documents

- Dail, D., and Madsen, L., 2011, Models for estimating abundance from repeated counts of an open metapopulation: Biometrics, v. 67, p. 577-587.

- Fiske, I., and Chandler, R.B., 2011, Unmarked-An R package for fitting hierarchical models of wildlife occurrence and abundance: Journal of Statistical Software, v. 43, p. 1-23.

- MacKenzie, D.I., Nichols, J.D., Hines, J.E., Knutson, M.G., and Franklin, A.B., 2003, Estimating site occupancy, colonization, and local extinction when a species is detected imperfectly: Ecology, v. 84, p. 2,200-2,207.

- R Core Team, 2015, R-A language and environment for statistical computing: Vienna, Austria, R Foundation for Statistical Computing, accessed September 28, 2015, at http:/www.Rproject.org/.

- Royle, J.A., 2004, N-mixture models for estimating population size from spatially replicated counts: Biometrics, v. 60, p. 108-115.

3. Procedures for data analysis:

3.1. Foothill yellow-legged frogs occupancy analysis:

3.1.1. Within the database, run the query RABOccuSurveyl, limiting the year field to the current year. Export the result of the query to Excel $^{\circledR}$.

3.1.2. This file will have multiple lines for generalized linear random tessellation stratified (GRTS) units with multiple subhabitat units, and the lengths of these need to be summed.

Create a pivot table, add GRTS as a row, and Length and OCLength as values, and set the Value field setting as Sum. Add Breed, Date, WaterTemp, AirTemp, Wind, and Weather as values, and set the Value field setting as Maximum. Copy the pivot table and paste it into a new spreadsheet as values only. Create a new column named PctMC. For each GRTS unit, calculate $<$ Length/(Length + OCLength $)>$.

3.1.3. Within the database, run the query RABOOccuSurvey2, limiting the year field to the current year. Export the result to Excel. Again create a pivot table with GRTS as a Row. Add Breed, Date, WaterTemp, AirTemp, Wind, and Weather as values, set the Value field setting as Maximum. Copy the pivot table and paste it into a new spreadsheet as values only.

3.1.4. Appendix $\mathrm{J}$ shows an example of how the data are organized for analysis. Open the RABO_occu.csv file containing all previous years data. Insert two new columns after the last $\mathrm{y} . \mathrm{X}$ column and paste the presence/absence data (Breed) for survey one in the first new column and survey two in the second new column. Repeat with the data for Date, WaterTemp, AirTemp, Wind, and Weather. 
3.1.5. Insert one new column after the last column for GravelX, paste the length of habitat for each GRTS unit, and repeat for PctMc. Update all column headers. Save the file as a comma separated values (.csv) file.

3.1.6. Appendix L contains the R script used to conduct a multi-season occupancy model.

3.1.7. Qualified personnel with statistical and occupancy modeling experience should run the scripts and evaluate the model output to assure adequate convergence.

3.2. Western pond turtle occupancy analysis:

3.2.1. Within the database, run the query $A C M A о с c u$, limiting the year field to the current year. Export the result of the query to Excel. Sort the new Excel spreadsheet by HabitatType. Cut and paste the pond data into a separate datasheet, repeat all steps for the main stem data on the pond data. Return to the main stem (MC) data and sort first by Survey, then by GRTS number.

3.2.2. Appendix $\mathrm{K}$ shows an example of how the data are organized for analysis. Open the ACMA_occu.csv file containing all previous years data. Ensure these data are sorted by GRTSID. Insert three new columns after the last $y . X$ column and paste in the presence/absence data (TurtNum) for survey one in the first new column, survey two in the second new column, and survey three in the third new column. Repeat with the data for

Date, WaterTemp, AirTemp, Wind, and Weather. Insert one new column after the VegetationL.X column and paste in the current year data. Repeat for VegetationR, BankL, BankR, BaskingL, BaskingR, VelocityL, VelocityR, DepthL, and DepthR.

3.2.3. After the current year data are added, save the spreadsheet as a comma separated values (.csv) file.

3.2.4. Appendix $\mathrm{M}$ contains the $\mathrm{R}$ script used to conduct a multi-season occupancy model.

3.2.5. Appendix $\mathrm{N}$ contains the $\mathrm{R}$ script used to conduct a multi-season open-population abundance model.

4. Reporting

4.1. Basic parameter estimates (mean and variance) for occupancy, abundance, and probability of detection will be reported annually. Every 5 years the model structure, assumptions and covariates will be reassessed, the R script will be updated as needed, and these changes will be reported to the Trinity River Restoration Program (TRRP). 
Standard Operating Procedure (SOP) 7: Revising the Protocol

Revision History Log:

\begin{tabular}{c|c|c|c|c|c}
\hline $\begin{array}{c}\text { Previous } \\
\text { Version No. }\end{array}$ & $\begin{array}{c}\text { Revision } \\
\text { Date }\end{array}$ & Author & Changes Made & Reasons for Change & $\begin{array}{c}\text { New Version } \\
\text { No. }\end{array}$ \\
\hline 1.0 & & & & & \\
\hline & & & & & \\
\hline & & & & & \\
\hline
\end{tabular}

1. Purpose

This Standard Operating Procedure (SOP) describes how to make changes to this report and SOPs, and the methods to track these changes. The procedures outlined in this SOP should be followed to avoid confusion in how data are collected and analyzed. All personnel involved with the project should be familiar with this SOP to ensure the most current methodologies are being used.

2. Reference Documents

- None

3. Procedures and General Requirements

3.1. This report and accompanying SOPs were developed to provide current and sound methodologies for collecting and analyzing data for foothill yellow-legged frogs and western pond turtles. However, any of these documents may require editing as new information becomes available or priorities change.

3.2. Any edits should be peer-reviewed at an appropriate level. All edits should be reviewed for clarity and technical correctness.

3.3. All edits and protocol version numbering should be approved by the project lead. Once approved changes should be documented in the appropriate Revision History Log table. Number minor changes incrementally by tenths, for example the first minor revision would be numbered 1.1. Major revisions should be numbered with the next whole number (version no. $2.0,3.0$,and so on). Details on date of revision, author, exact location of revisions, and reason for revision should be recorded in the Revision History Log table.

3.4. Ensure new copies of the protocol are circulated as appropriate. 


\section{Acknowledgments}

This study was made possible in part by funding from the Trinity River Restoration Program (TRRP). The TRRP is a cooperative program of the Bureau of Reclamation, U.S. Fish and Wildlife Service, U.S. Forest Service, National Oceanic and Atmospheric Administration Fisheries, California Department of Fish and Wildlife, Trinity County, and the Hoopa Valley and Yurok Tribes. Additional funding was provided by the U.S. Geological Survey's Amphibian Research and Monitoring Initiative (ARMI). This is product 521 of the Amphibian Research and Monitoring Initiative.

\section{References Cited}

Ashton, D.T., Bettaso, J.B., and Welsh, H.H., Jr., 2010, Comparative ecology of western pond turtle (Actinemys marmorata) populations on the free-flowing South Fork and regulated Main Fork Trinity River - demography, size and body condition comparisons, thermal ecology and spatial dynamics: Final Report to the Trinity River Restoration Program, 55 p., accessed November 4, 2015, at http://www.odp.trrp.net/Data/Documents/Details.aspx?document=1217.

Ashton, D.T., Bettaso, J.B., and Welsh, H.H., Jr., 2015, Changes across a decade in size, growth, and body condition of western pond turtle (Actinemys marmorata) populations on free-flowing and regulated forks of the Trinity River in northwest California: Copeia, v. 103, p. 621-633.

Ben-Ezra, E., Bulte, G., and Blouin-Demers, G., 2008, Are locomotor performances coadapted to preferred basking temperature in the northern map turtle (Graptemys geographica)?: Journal of Herpetology, v. 42, p. 322-331.

Bury, R.B., Welsh, H.W., Jr., Germano, D.J., and Ashton, D.T., eds., 2012, Western pond turtlebiology, sampling techniques, inventory and monitoring, conservation, and management: Northwest Fauna Number 7, Society for Northwestern Vertebrate Biology, Olympia, 128 p.

California Department of Fish and Game, Hoopa Valley Tribe, U.S. Fish and Wildlife Service and Yurok Tribe, 2010, Interdisciplinary habitat assessment plan of the upper Trinity River-Work Plan FY 2010: Prepared for Trinity River Restoration Program,Weaverville, California, 43 p.

Dail, D., and Madsen, L., 2011, Models for estimating abundance from repeated counts of an open metapopulation: Biometrics, v. 67, p. 577-587.

Fiske, I., and Chandler, R.B., 2011, Unmarked-An R package for fitting hierarchical models of wildlife occurrence and abundance: Journal of Statistical Software, v. 43, p. 1-23.

Gosner, K.L., 1960, A simplified table for staging anuran embryos and larvae with notes on identification: Herpetologica, v. 16, p. 183-190.

Jennings, M.R., and Hayes, M.P., 1994, Amphibian and reptile species of special concern in California: Final report submitted to the California Department of Fish and Game, 260 p., accessed November 4, 2015, at http://www.dfg.ca.gov/wildlife/nongame/ssc/amphibian-reptile.html.

Lind, A.J., Welsh, H.H., Jr., and Wilson, R.A., 1996, The effects of a dam on breeding habitat and egg survival of the foothill yellow-legged frog (Rana boylii) in northwestern California: Herpetological Review, v. 27, p. 62-67.

MacKenzie, D.I., Nichols, J.D., Hines, J.E., Knutson, M.G., and Franklin, A.B., 2003, Estimating site occupancy, colonization, and local extinction when a species is detected imperfectly: Ecology, v. 84, p. 2,200-2,207.

Oakley, K.A., Thomas, L.P., and Fancy, S.G., 2003, Guidelines for long-term monitoring protocols: Wildlife Society Bulletin, v. 31, p. 1,000-1,003.

Peterman, W.E., Rittenhouse, T.A.G., Earl, J.E., and Semlitsch, R.D., 2013, Demographic network and multi-season occupancy modeling on Rana sylvatica reveal spatial and temporal patterns of population connectivity and persistence: Landscape Ecology, v. 28, p. 1,601-1,613. 
Phillott, A.D., Speare, R., Hines, H.B., Skerratt, L.F., Meyer, E., McDonald, K.R., Cashings, S.D., Mendez, D., and Berger, L., 2010, Minimising exposure of amphibians to pathogens during field studies: Diseases of Aquatic Organisms, v. 92, nos. 2-3, p. 175-185.

$\mathrm{R}$ Core Team, 2015, R-A language and environment for statistical computing: Vienna, Austria, $\mathrm{R}$ Foundation for Statistical Computing, accessed September 28, 2015, at http://www.R-project.org/. Reese, D.A., and Welsh, H.H., Jr., 1998a, Comparative demography of Clemmys marmorata populations in the Trinity River of California in the context of dam-induced alterations: Journal of Herpetology, v. 32, p. 505-515.

Reese, D.A., and Welsh, H.H. Jr., 1998b, Habitat use by western pond turtles in the Trinity River, California: Journal of Wildlife Management, v. 62, p. 842-853.

Royle, J.A., 2004, N-mixture models for estimating population size from spatially replicated counts: Biometrics, v. 60, p. 108-115.

Sloan, L.M., 2012, Population structure, life history, and terrestrial movements of western pond turtles (Actinemys marmorata) in lentic habitats along the Trinity River, California: Master of Science Thesis, Humboldt State University, Arcata, California, 111 p., accessed November 4, 2015, at http://odp.trrp.net/Data/Documents/Details.aspx?document=1819.

Snover, M.L., Adams, M.J., Ashton, D.T., Betasso, J., and Welsh, H.H., Jr., 2015, Evidence of countergradient growth in western pond turtles (Actinemys marmorata) across thermal gradients. Freshwater Biology, v. 60, p. 1,944-1,963.

U.S. Department of the Interior, 2000, Record of decision-Trinity River mainstem fishery restoration: Final Environmental Impact Statement/Environmental Impact Report, 43 p., accessed November 4, 2015, at http://www.odp.trrp.net/Data/Documents/Details.aspx?document=227.

U.S. Fish and Wildlife Service, 2015a, Endangered and threatened wildlife and plants; 90-day findings on 10 petitions: Federal Register, v. 80, no. 69, p. 19,259.

U.S. Fish and Wildlife Service, 2015b, Endangered and threatened wildlife and plants; 90-day findings on 31 petitions: Federal Register, v. 80, no. 126, p. 37,568.

U.S. Fish and Wildlife Service and Hoopa Valley Tribe, 1999, Trinity River flow evaluation - final report: Report to the Secretary of the U.S. Department of the Interior, 513 p., accessed November 4, 2015 at http://odp.trrp.net/Data/Documents/Details.aspx?document=226.

U.S. Geological Survey, 2001, National Hydrography Dataset (NHD): U.S. Geological Survey, accessed September 28, 2015, at http://www.nhd.usgs.gov.

Wheeler, C.A., Bettaso, J.B., Ashton, D.T., and Welsh, H.H., Jr., 2013, Effects of water temperature on breeding phenology, growth and timing of metamorphosis of foothill-yellow-legged frogs (Rana boylii) on the mainstem and selected tributaries of California's Trinity River-2004-2009: Final Report to the Trinity River Restoration Program, 37 p., accessed November 4, 2015, at http://odp.trrp.net/Data/Documents/Details.aspx?document=2165.

Wheeler, C.A., Bettaso, J.B., Ashton, D.T., and Welsh, H.H., Jr., 2014, Effects of water temperature on breeding phenology, growth and metamorphosis of foothill-yellow-legged frogs (Rana boylii) —a case study of the regulated mainstem and unregulated tributaries of California's Trinity River: River Research and Applications, v. 31, no. 10, p. 1,276-1,286, doi: 10.1002/rra.2820. 

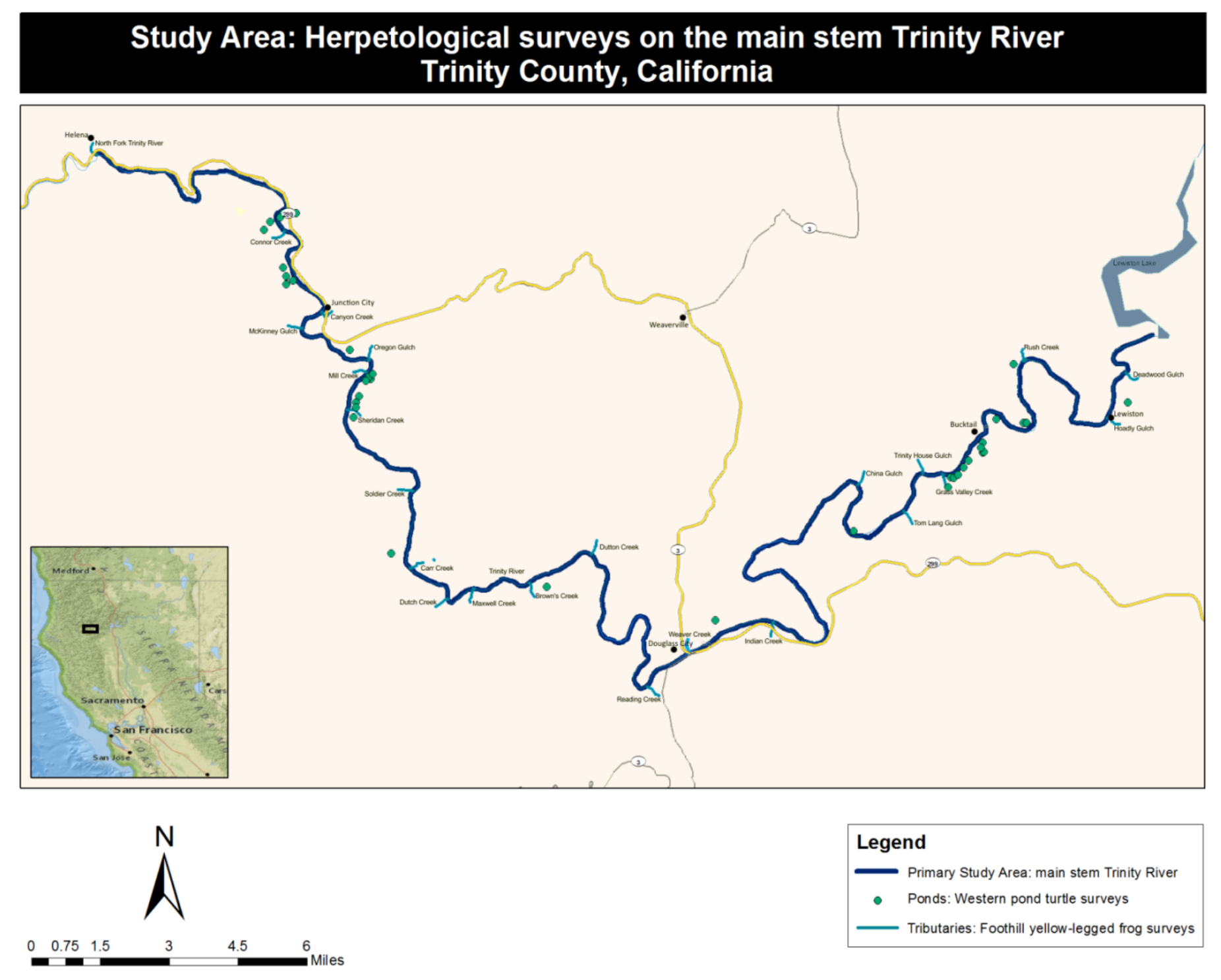

Figure 1. Study area for herpetological surveys on main stem Trinity River from the Lewiston Dam to the confluence with the North Fork Trinity River, Trinity County, California. Inset map shows the Northern California location of the study area in Trinity County. Perennial ponds that were surveyed for western pond turtles are within 500 meters of the center line of the river. Tributaries that were surveyed for foothill yellow-legged frogs are those that contain frog breeding habitat. In 2014 and 2015, foothill yellow-legged frog surveys were limited to downstream Steel Bridge River Access. 


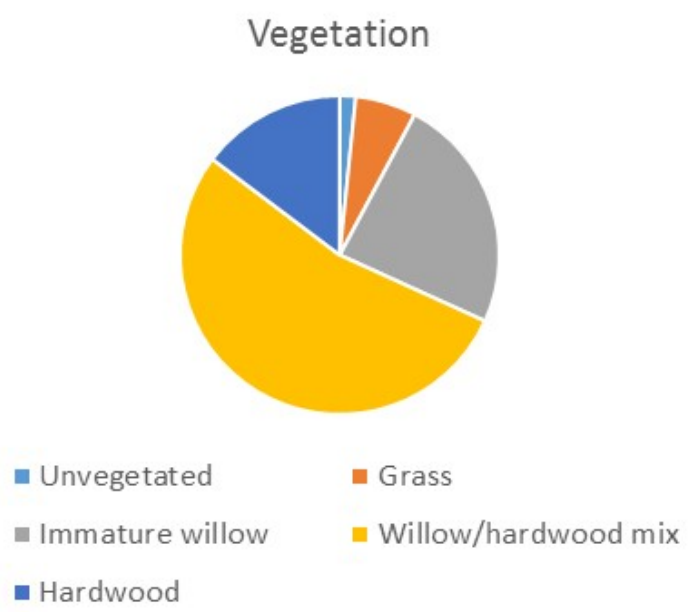

Basking habitat

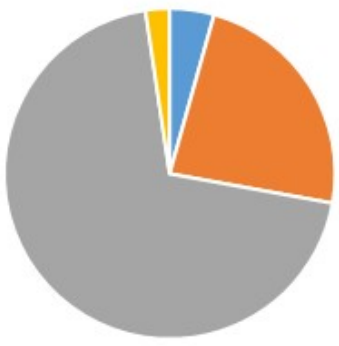

- No bask

- Few bask

n Moderate bask w High Bask
Bank structure

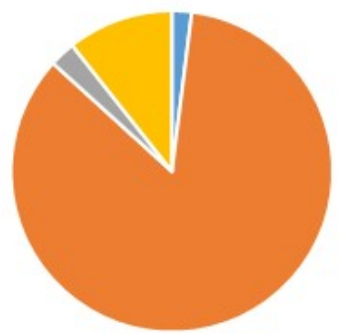

- Cut bank,no veg $\quad$ Cut bank, roots

n Sloped bank,unveg $=$ Sloped bank, veg

Water velocity

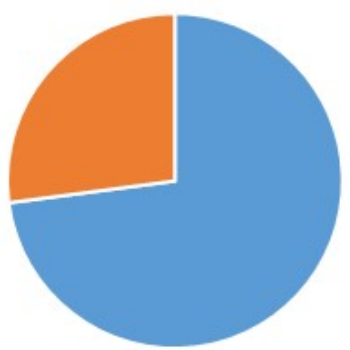

- No visable current $\quad$ Visibile current

Water depth

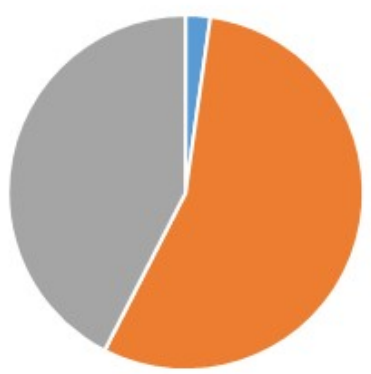

- Less than $10 \mathrm{~cm} \quad \square 10-100 \mathrm{~cm}$

- Greater than $100 \mathrm{~cm}$

Figure 2. Summary of habitat characteristics for 808 basking western pond turtle (Actinemys marmorata) sightings along the Trinity River, Trinity County, California, 2013-15. 


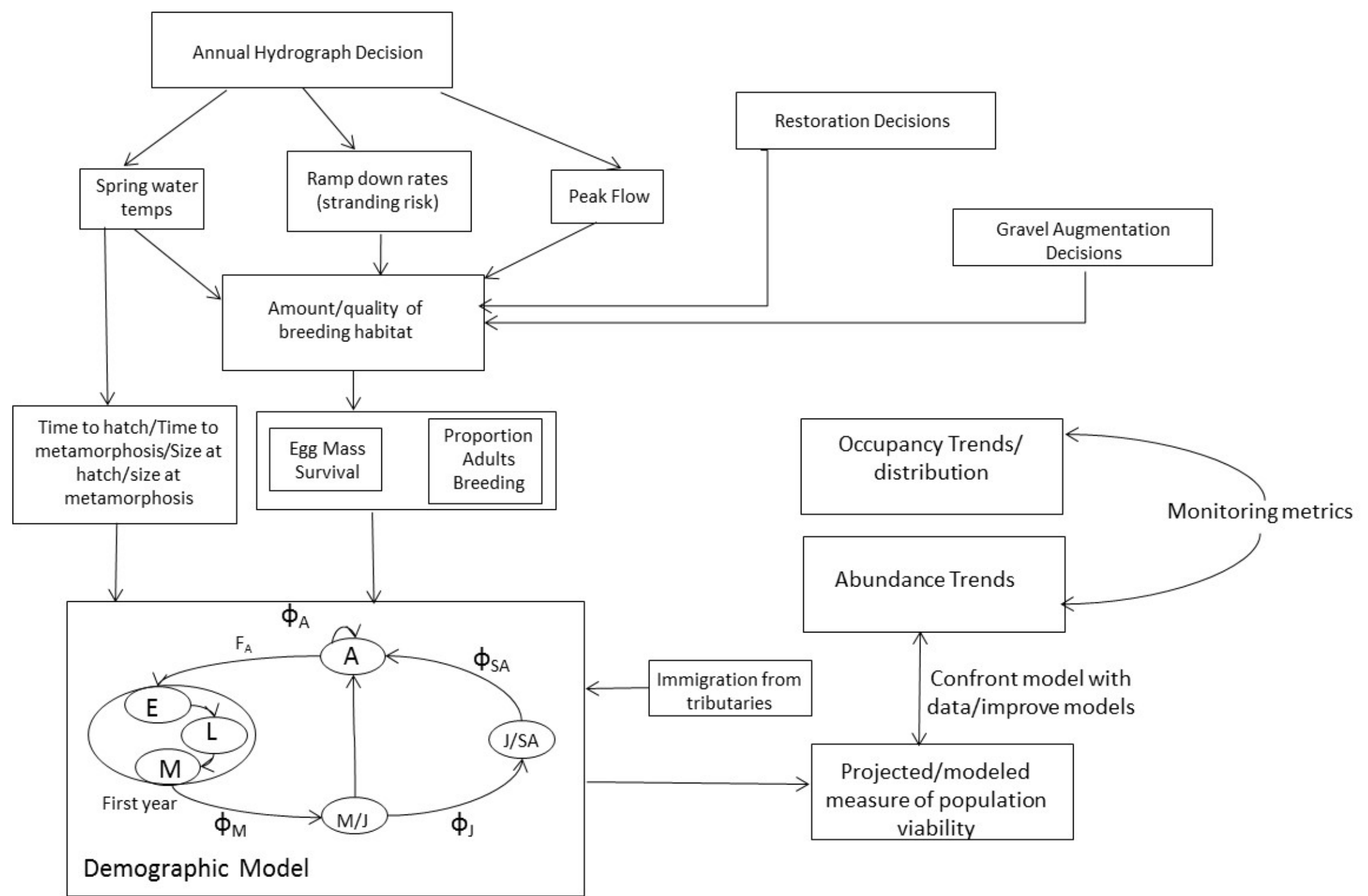

Figure 3. Conceptual model for the links between management decisions and population processes for foothill yellow-legged frogs (Rana boylii) on the Trinity River, Trinity County, California. A decision support tool would be composed of a population model that estimates the impacts of management decisions on the frog population. Data from continued monitoring and targeted research questions can be used to confront and refine the population model and to determine if decisions are achieving objectives. 


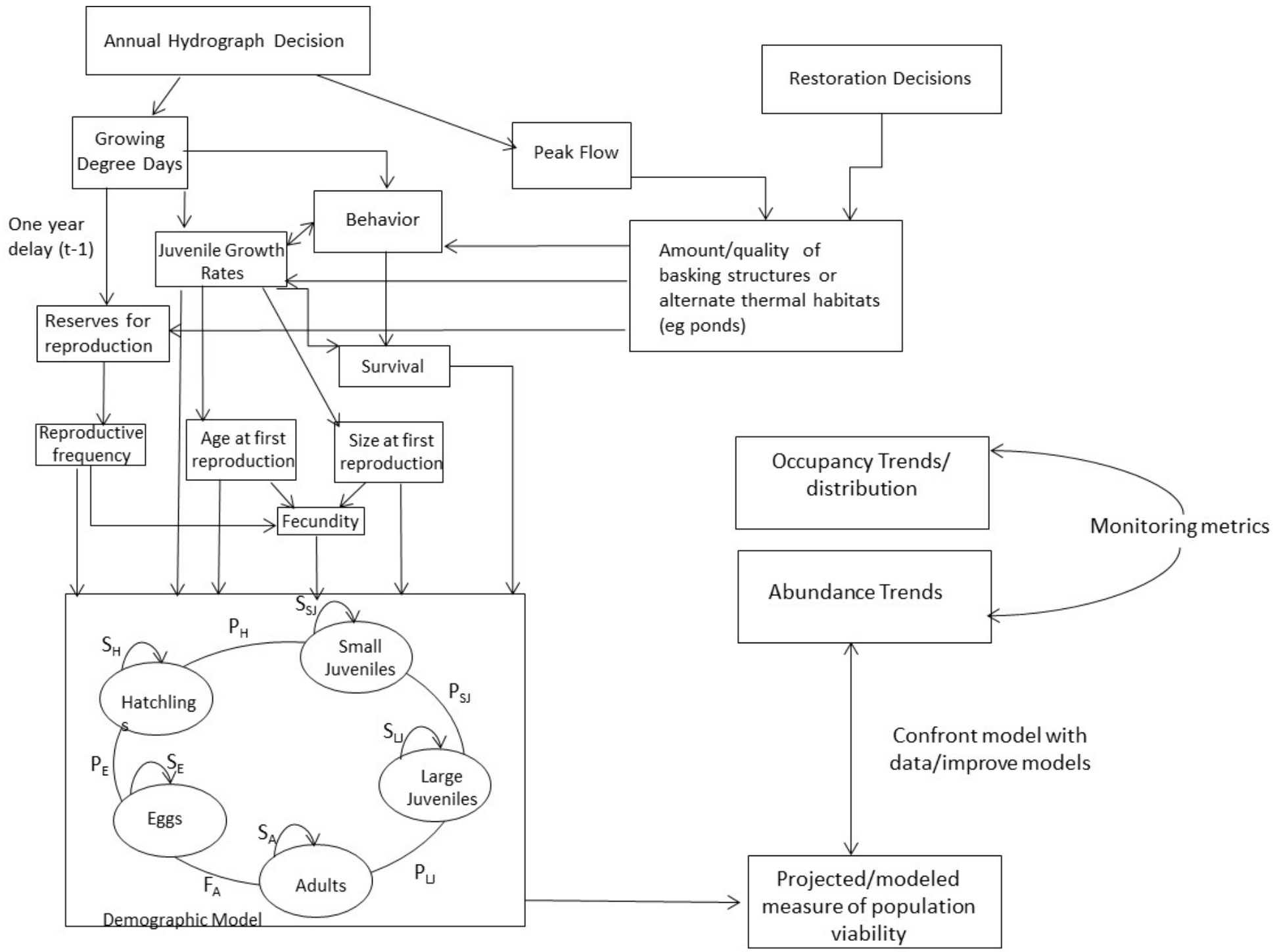

Figure 4. Conceptual model for the links between management decisions and population processes for western pond turtles (Actinemys marmorata) on the Trinity River, Trinity County, California. A decision support tool would be composed of a population model that estimates the impacts of management decisions on the turtle population. Data from continued monitoring and targeted research questions can be used to confront and refine the population model and to determine if decisions are achieving objectives. 


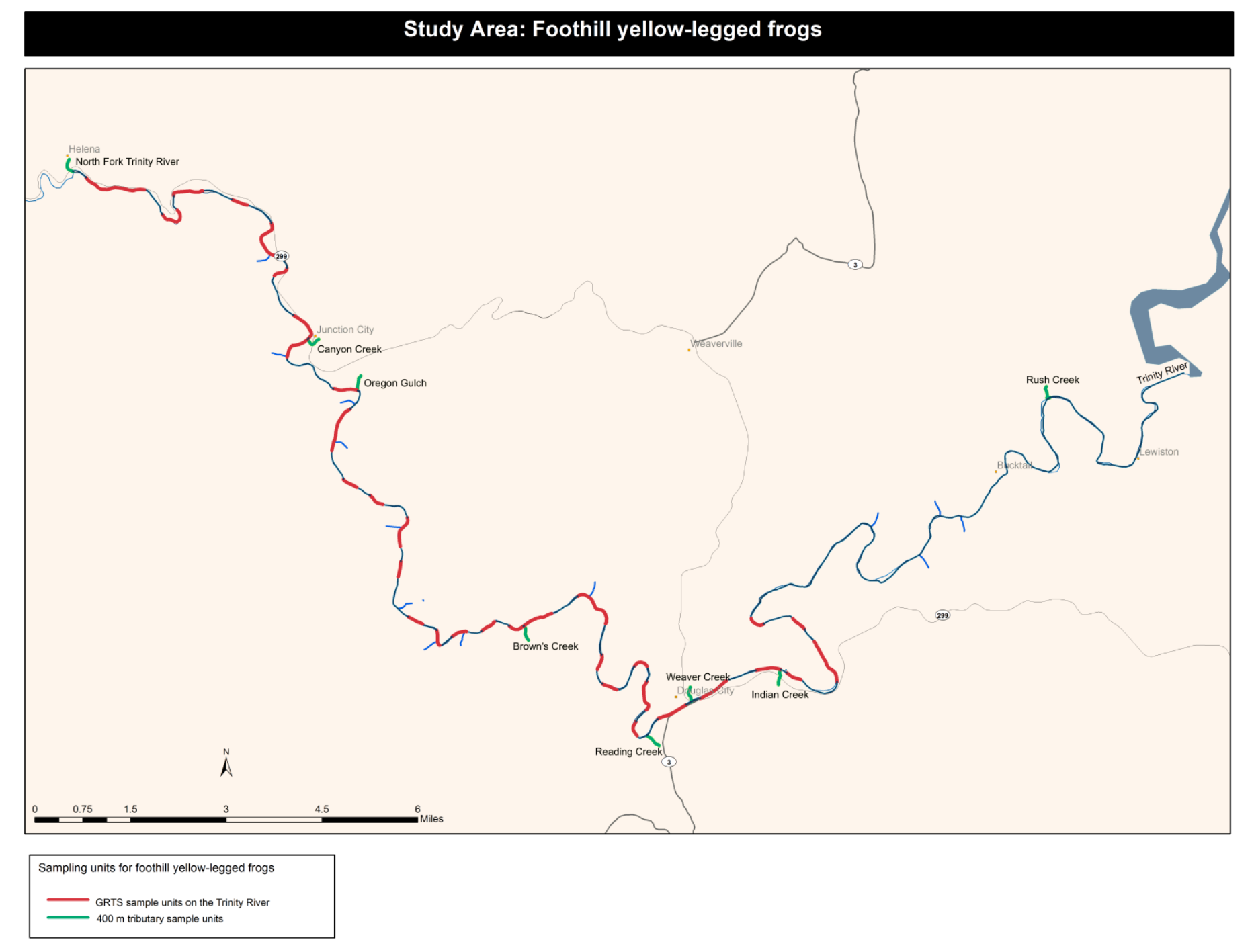

Figure 5. Sample sites for foothill yellow-legged frogs (Rana boylii) from the Lewiston Dam to the confluence with the North Fork Trinity River, Trinity County, California, 2013-15. Survey units for both the main stem Trinity River and the tributaries are $400 \mathrm{~m}$ in length. 


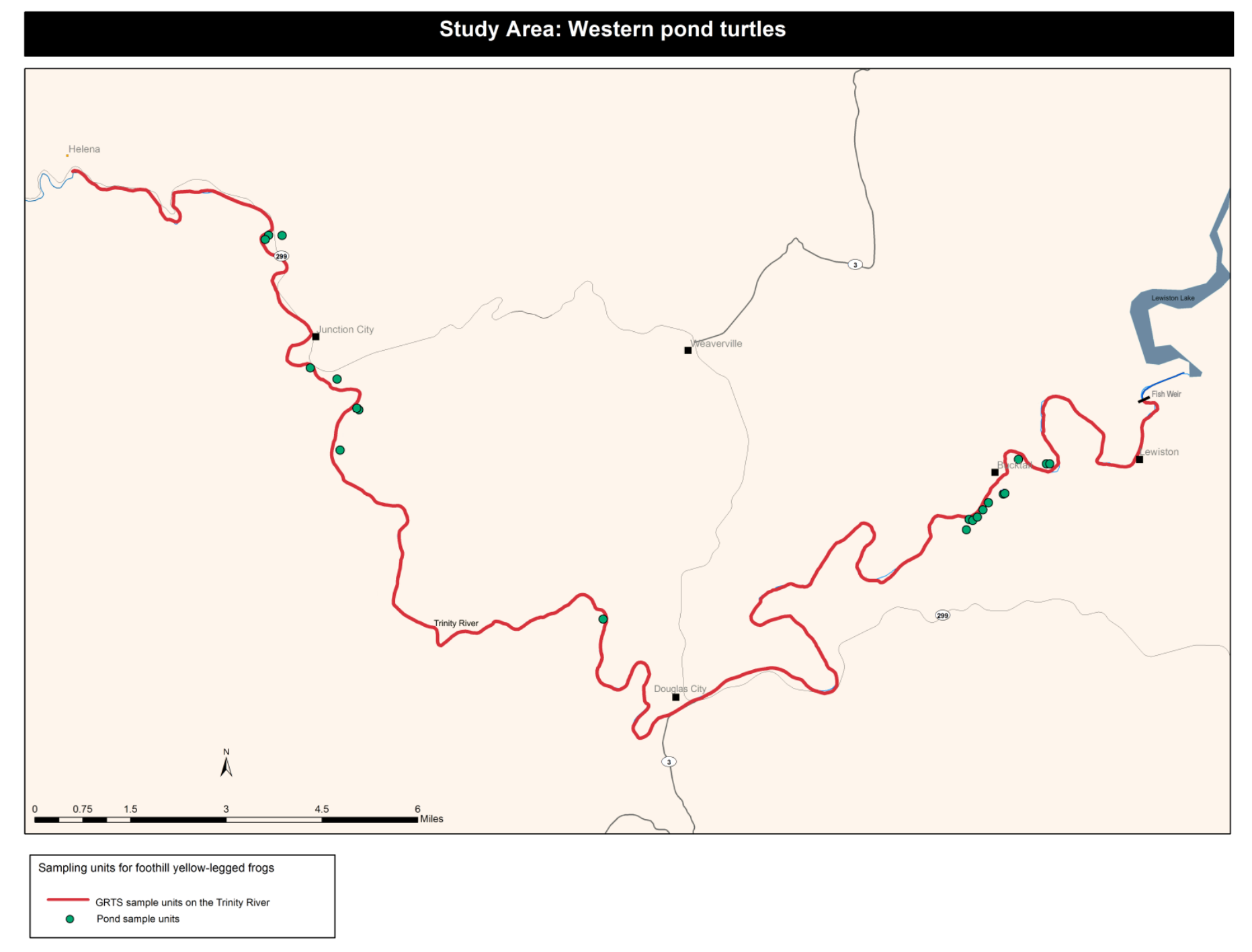

Figure 6. Sample sites for western pond turtle (Actinemys marmorata) surveys from the fish weir to the confluence with the North Fork Trinity River, Trinity River, Trinity County, California, 2013-15. An individual river sample site is defined as a $400 \mathrm{~m}$ stretch based on the river center line; all 156 potential river sample sites within the study area were surveyed. 

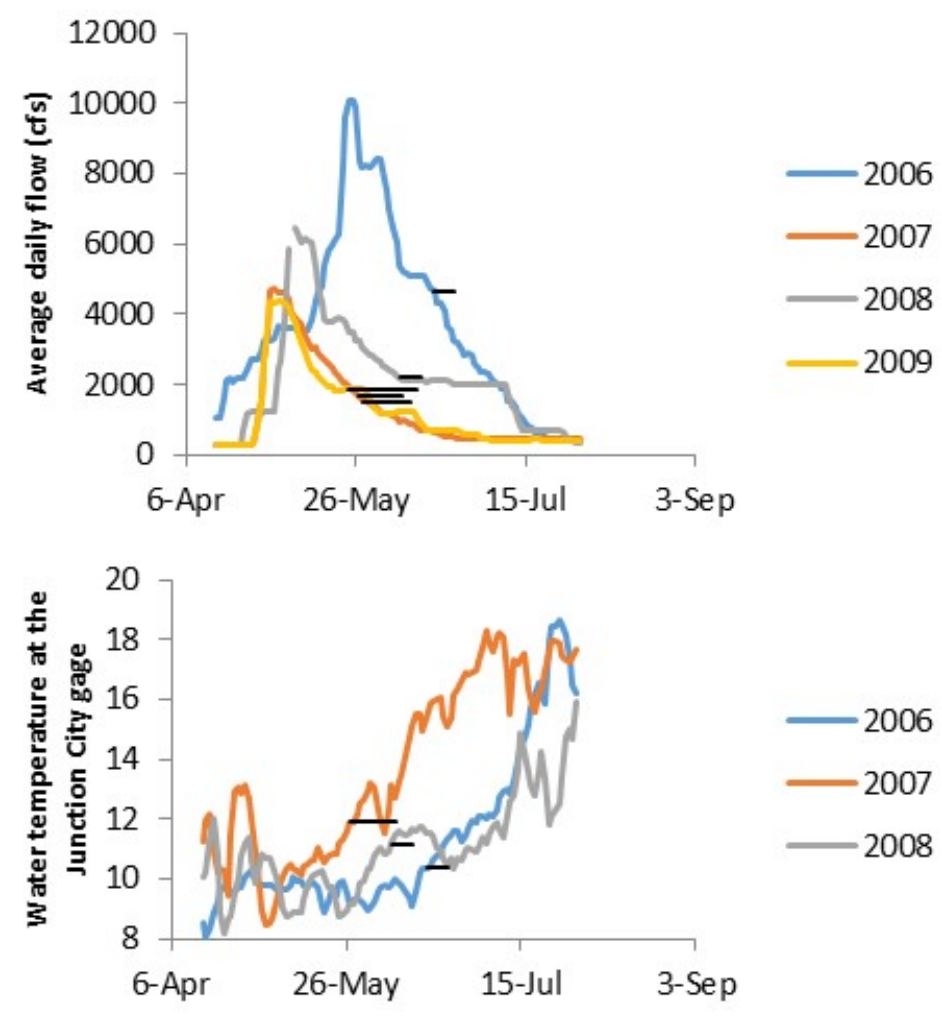

Figure 7. Initiation and duration of foothill yellow-legged frog (Rana boylii) breeding activity as it relates to flow and water temperature, Trinity River, Trinity County, California, 2006-09. (Top graph) Annual spring/summer hydrographs from 2006 to 2009 for the Trinity River based on flow rates at the Lewiston Dam. (Bottom graph) Spring and summer water temperatures from the Junction City (station 11526250, USGS California Water Science Center) streamgage from 2006 to 2008 . Horizontal lines show the start and duration of breeding for each year (Wheeler and others, 2014). 
Table 1. Multi-season occupancy models describing the probability that a site was occupied by breeding foothill yellow-legged frogs (Rana boylii), Trinity River, Trinity County, California, 2013-15.

[Models: indicates the covariates included with the extinction term $(\varepsilon)$ in the models. The $\psi_{1}, \gamma$, and p terms are as described for the global model in the text. Abbreviations: K, number of parameters; AICc, Akaike Information Criterion for small sample size; $\triangle \mathrm{AICc}$, difference between the AICc of the current model and the top ranked model; ModelLik, model likelihood; AICcWt, model weight; LL, log likelihood; CumWt, cumulative weight of models]

\begin{tabular}{lrrrcccc}
\hline \multicolumn{1}{c}{ Models } & K & AICc & AAICc & ModelLik & AICcWt & LL & CumWt \\
\hline Pctmc & 9 & 311.24 & 0.00 & 1.00 & 0.78 & -145.69 & 0.76 \\
Distance & 9 & 315.85 & 4.61 & 0.10 & 0.08 & -148.00 & 0.94 \\
Global & 11 & 316.00 & 4.76 & 0.09 & 0.07 & -145.61 & 0.87 \\
Constant & 8 & 316.79 & 5.55 & 0.06 & 0.05 & -149.66 & 0.98 \\
Gravel & 9 & 318.79 & 7.55 & 0.02 & 0.02 & -149.47 & 1.00 \\
Null & 4 & 352.54 & 41.29 & 0.00 & 0.00 & -172.07 & 1.00 \\
\hline
\end{tabular}


Table 2. Occupancy model results of yellow-legged frogs (Rana boylii) and western pond turtles (Actinemys marmorata), Trinity River, Trinity County, California, 2013-15 using data from all generalized linear random tessellation stratified sampling (GRTS) panels and data from five-panel GRTS only.

[For each model, colonization was held constant, $\gamma($.). Standard error is shown in parenthesis below parameter estimates. Abbreviations: $p$, probability of detection; $\psi$, site occupancy; $\varepsilon$, probability of extinction]

\begin{tabular}{|c|c|c|c|c|c|c|c|c|c|c|}
\hline & \multirow{2}{*}{ Model } & \multicolumn{9}{|c|}{ Parameter estimates from best models based on $\mathrm{AlC}_{\mathrm{c}}$} \\
\hline & & p 2013 & p 2014 & p 2015 & $\Psi 2013$ & $\Psi 2014$ & $\Psi 2015$ & $\varepsilon 2014$ & $\varepsilon 2015$ & Y \\
\hline \multicolumn{11}{|c|}{ Foothill yellow-legged frogs } \\
\hline All GRTS & $\psi($ length,distUP), $\varepsilon($ pctmc $), p($ year $)$ & $\begin{array}{c}0.27 \\
(0.072)\end{array}$ & $\begin{array}{c}0.66 \\
(0.074)\end{array}$ & $\begin{array}{c}0.87 \\
(0.077)\end{array}$ & $\begin{array}{c}0.31 \\
(0.162)\end{array}$ & $\begin{array}{c}0.22 \\
(0.047)\end{array}$ & $\begin{array}{c}0.17 \\
(0.040)\end{array}$ & $\begin{array}{c}0.25 \\
(0.054)\end{array}$ & $\begin{array}{c}0.26 \\
(0.059)\end{array}$ & $\begin{array}{c}0.02 \\
(0.027)\end{array}$ \\
\hline Panel GRTS & $\psi($ length,distUP), $\varepsilon$ (pctmc),p(year) & $\begin{array}{c}0.32 \\
(0.087)\end{array}$ & $\begin{array}{c}0.83 \\
(0.080)\end{array}$ & $\begin{array}{c}1.00 \\
(0.001)\end{array}$ & $\begin{array}{c}0.35 \\
(0.194)\end{array}$ & $\begin{array}{c}0.20 \\
(0.054)\end{array}$ & $\begin{array}{c}0.12 \\
(0.050)\end{array}$ & $\begin{array}{c}0.33 \\
(0.034)\end{array}$ & $\begin{array}{c}0.36 \\
(0.032)\end{array}$ & $\begin{array}{c}0.00 \\
(0.000) \\
\end{array}$ \\
\hline \multicolumn{11}{|c|}{ Western pond turtles } \\
\hline All GRTS & $\begin{array}{l}\psi(\text { bask,dist }), \varepsilon(.), p(\text { date,Idate,air,Iair,water, } \\
\text { year, bank,bask,vel,distance) }\end{array}$ & $\begin{array}{c}0.55 \\
(0.016)\end{array}$ & $\begin{array}{c}0.51 \\
(0.015)\end{array}$ & $\begin{array}{c}0.44 \\
(0.015)\end{array}$ & $\begin{array}{c}0.39 \\
(0.064)\end{array}$ & $\begin{array}{c}0.51 \\
(0.047)\end{array}$ & $\begin{array}{c}0.59 \\
(0.054)\end{array}$ & $\begin{array}{c}0.087 \\
(0.038)\end{array}$ & - & $\begin{array}{c}0.25 \\
(0.042)\end{array}$ \\
\hline Panel GRTS & $\begin{array}{l}\psi(\text { bask,dist }), \varepsilon(.), p(\text { date,Idate,air,Iair,water, } \\
\text { year, bank,bask,vel,distance) }\end{array}$ & $\begin{array}{c}0.48 \\
(0.024)\end{array}$ & $\begin{array}{c}0.52 \\
(0.023)\end{array}$ & $\begin{array}{c}0.52 \\
(0.024)\end{array}$ & $\begin{array}{c}0.48 \\
(0.133)\end{array}$ & $\begin{array}{c}0.49 \\
(0.090)\end{array}$ & $\begin{array}{c}0.50 \\
(0.093)\end{array}$ & $\begin{array}{c}0.15 \\
(0.059)\end{array}$ & - & $\begin{array}{c}0.17 \\
(0.070)\end{array}$ \\
\hline Ponds & $\psi(),. \varepsilon(),. \mathrm{p}()$. & $\begin{array}{c}0.71 \\
(0.045)\end{array}$ & - & - & $\begin{array}{c}0.77 \\
(0.112)\end{array}$ & $\begin{array}{c}0.70 \\
(0.094)\end{array}$ & $\begin{array}{c}0.66 \\
(0.102)\end{array}$ & $\begin{array}{c}0.15 \\
(0.073)\end{array}$ & - & $\begin{array}{c}0.23 \\
(0.147)\end{array}$ \\
\hline
\end{tabular}


Table 3. Multi-season occupancy models describing the probability that a site was occupied by basking western pond turtles (Actinemys marmorata), Trinity River, Trinity County, California, 2013-15.

[Models indicates the covariates included with the extinction term $(\varepsilon)$ in the models. The $\psi_{1}, \gamma$, and p terms are as described for the global model in the text. Abbreviations: K, number of parameters; $\mathrm{AIC}_{\mathrm{c}}$, Akaike Information Criterion for small sample size; $\triangle \mathrm{AIC}_{\mathrm{c}}$, difference between the $\mathrm{AIC}_{\mathrm{c}}$ of the current model and the top ranked model; ModelLik, model likelihood; AICcWt, model weight; LL, log likelihood; CumWt, cumulative weight of models]

\begin{tabular}{lccccccc}
\hline \multicolumn{1}{c}{ Models } & K & AICc & AAICc & ModelLik & AICcWt & LL & CumWt \\
\hline Constant & 23 & 1242.81 & 0.00 & 1.00 & 0.91 & -594.223 & 0.91 \\
Global & 28 & 1248.26 & 5.45 & 0.95 & 0.06 & -589.738 & 0.97 \\
Bank & 23 & 1251.50 & 8.69 & 0.17 & 0.01 & -598.57 & 0.98 \\
Distance & 22 & 1251.58 & 8.77 & 0.13 & 0.01 & -599.985 & 0.99 \\
Velocity & 23 & 1253.83 & 11.02 & 0.09 & 0.00 & -599.735 & 1.00 \\
Bask & 23 & 1253.96 & 11.15 & 0.01 & 0.00 & -599.8 & 1.00 \\
Null & 4 & 1282.09 & 39.28 & 0.00 & 0.00 & -636.914 & 1.00 \\
\hline
\end{tabular}

Table 4. Multi-season occupancy models describing the probability that a site was occupied by basking western pond turtles (Actinemys marmorata) observed in ponds adjacent to the Trinity River, Trinity County, California, 2013-15.

[Model indicates the covariates included with the extinction term $(\varepsilon)$ in the models. The $\psi_{1}, \gamma$, and $p$ terms are as described for the global model in the text. Abbreviations: $\mathrm{K}$, number of parameters; $\mathrm{AIC}_{\mathrm{c}}$, Akaike Information Criterion for small sample size; $\triangle \mathrm{AIC}_{\mathrm{c}}$, difference between the $\mathrm{AIC}_{\mathrm{c}}$ of the current model and the top ranked model; ModelLik, model likelihood; AICcWt, model weight; LL, log likelihood; CumWt, cumulative weight of models]

\begin{tabular}{lccccccc}
\hline \multicolumn{1}{c}{ Models } & K & AIC & DAICc & ModelLik & AICcWt & LL & CumWt \\
\hline Null & 4 & 208.55 & 0.00 & 1.00 & 0.99 & -98.94 & 0.99 \\
Constant & 7 & 219.29 & 10.74 & 0.00 & 0.00 & -97.98 & 1.00 \\
Bask & 8 & 221.75 & 13.20 & 0.00 & 0.00 & -96.33 & 1.00 \\
Depth & 8 & 223.70 & 15.15 & 0.00 & 0.00 & -97.30 & 1.00 \\
Bank & 8 & 224.98 & 16.43 & 0.00 & 0.00 & -97.94 & 1.00 \\
Vegetation & 8 & 225.03 & 16.48 & 0.00 & 0.00 & -97.97 & 1.00 \\
Global & 11 & 245.66 & 37.12 & 0.00 & 0.00 & -95.33 & 1.00 \\
\hline
\end{tabular}




\section{Appendixes}

\section{Appendix A. Example of Field Schedule Based on the 2015 Season}

\begin{tabular}{|c|c|c|c|}
\hline Date & Put-in & Take-out & Itinerary \\
\hline \multicolumn{4}{|c|}{ Foothill yellow-legged frog surveys } \\
\hline 28-April-15 & - & - & $1^{\text {st }}$ survey Oregon Gulch, Weaver Creek, Indian Creek \\
\hline 29-April-15 & - & - & $\begin{array}{l}2^{\text {nd }} \text { survey Oregon Gulch, Weaver Creek, Indian } \\
\text { Creek }\end{array}$ \\
\hline 27-May-15 & - & - & $\begin{array}{l}\text { Frog survey technique training; } 1^{\text {st }} \text { survey North Fork } \\
\text { and Canyon Creek }\end{array}$ \\
\hline 28-May-15 & Rush/Douglas City & $\begin{array}{l}\text { Bucktail/Douglas } \\
\text { City Campground }\end{array}$ & $\begin{array}{l}\text { Kayak training, } 1^{\text {st }} \text { survey Rush and Reading Creeks, } \\
\text { Douglas City Backwater }\end{array}$ \\
\hline 29-May-15 & $\begin{array}{l}\text { Douglas City } \\
\text { Campground }\end{array}$ & Evan's Bar & $\begin{array}{l}2^{\text {nd }} \text { survey Douglas City Backwater; } 1^{\text {st }} \text { survey } \\
\text { Brown's Creek; Canyon Creek after take-out }\end{array}$ \\
\hline 30-May-15 & - & - & 2nd survey North Fork and Canyon Creek \\
\hline 1-Jun-15 & Rush/Douglas City & $\begin{array}{l}\text { BucktailDouglas } \\
\text { City Campground }\end{array}$ & $\begin{array}{l}2^{\text {nd }} \text { survey Rush and Reading Creeks, Douglas City } \\
\text { Backwater }\end{array}$ \\
\hline 2-Jun-15 & Dutton & Evan’s Bar & $\begin{array}{l}2^{\text {nd }} \text { survey Brown's Creek; catch any missed sights or } \\
\text { work on data }\end{array}$ \\
\hline 9-Jun-15 & Sky Ranch & Bagdad & $1^{\text {st }}$ survey GRTS $128-143$ \\
\hline 10-Jun-15 & $\begin{array}{l}\text { Junction City } \\
\text { Campground }\end{array}$ & Bagdad & $\begin{array}{l}\text { starting where left of previous day; } 1^{\text {st }} \text { survey GRTS } \\
144-159\end{array}$ \\
\hline 11-Jun-15 & Evan’s Bar & $\begin{array}{l}\text { Junction City } \\
\text { Campground }\end{array}$ & $\begin{array}{l}1^{\text {st }} \text { survey GRTS } 111 \text { to } 127 ; 2^{\text {nd }} \text { survey GRTS } 128- \\
137\end{array}$ \\
\hline 12-Jun-15 & Evans Bar & $\begin{array}{l}\text { Sky Ranch (or } \\
\text { JCCG or Bagdad) }\end{array}$ & $\begin{array}{l}2^{\text {nd }} \text { survey GRTS } 111 \text { to } 127 \text {; complete survey } 2 \\
\text { between GRTS } 111 \text { and } 159 ; \text { work on data if } \\
\text { finished early }\end{array}$ \\
\hline 14-Jun-15 & $\begin{array}{l}\text { Douglas City } \\
\text { Campground }\end{array}$ & Evan's Bar & $1^{\text {st }}$ survey GRTS 80 to 110 \\
\hline 15-Jun-15 & Indian Creek & $\begin{array}{l}\text { Douglas City } \\
\text { Campground }\end{array}$ & $1^{\text {st }}$ survey GRTS 67 to 79 \\
\hline 16-Jun-15 & $\begin{array}{l}\text { Douglas City } \\
\text { Campground }\end{array}$ & Evan's Bar & $2^{\text {nd }}$ survey GRTS 80 to 110 \\
\hline 17-Jun-15 & Indian Creek & $\begin{array}{l}\text { Douglas City } \\
\text { Campground }\end{array}$ & $2^{\text {nd }}$ survey GRTS 67 to 79 \\
\hline \multicolumn{4}{|c|}{ Western pond turtle surveys } \\
\hline 7-Jul-15 & $\begin{array}{l}\text { Douglas City } \\
\text { Campground }\end{array}$ & Bagdad & Turtle habitat surveys GRTS 80 to 159 \\
\hline 6-Jul-15 & Lewiston Bridge & $\begin{array}{l}\text { Douglas City } \\
\text { Campground }\end{array}$ & Turtle habitat surveys GRTS 4 to 79 \\
\hline 8-Jul-15 & Sky Ranch & Bagdad & $1^{\text {st }}$ survey GRTS 128 to 159 plus ponds in that stretch \\
\hline 9-Jul-15 & $\begin{array}{l}\text { Douglas City } \\
\text { Campground } \\
\end{array}$ & Sky Ranch & $\begin{array}{l}1^{\text {st }} \text { survey GRTS } 80 \text { to } 127 \text { plus ponds along Sky } \\
\text { Ranch }\end{array}$ \\
\hline 10-Jul-15 & Bucktail & $\begin{array}{l}\text { Douglas City } \\
\text { Campground }\end{array}$ & $1^{\text {st }}$ survey GRTS 27 to 79 \\
\hline 13-Jul-15 & Lewiston Bridge & Bucktail & $1^{\text {st }}$ survey GRTS 4 to 26 plus ponds; $1^{\text {st }}$ survey \\
\hline
\end{tabular}




\begin{tabular}{llll}
\hline \multicolumn{1}{c}{ Date } & \multicolumn{1}{c}{ Put-in } & \multicolumn{1}{c}{ Take-out } & \multicolumn{1}{c}{ Itinerary } \\
\hline & & & \multicolumn{1}{c}{ complete, all pond complete } \\
\hline $14-J u l-15$ & Dutton & Bagdad & $2^{\text {nd }}$ survey GRTS 94 to 159 \\
\hline $15-J u l-15$ & Steelbridge & Dutton & $2^{\text {nd }}$ survey GRTS 54 to 93 \\
\hline $16-J u l-15$ & Lewiston Bridge & Steel Bridge & $2^{\text {nd }}$ survey GRTS 4 to 53 \\
\hline $17-J u l-15$ & Dutton & Bagdad & $3^{\text {rd }}$ survey GRTS 94 to 159 \\
\hline $20-J u l-15$ & Steelbridge & Dutton & $3^{\text {rd }}$ survey GRTS 54 to 93 \\
\hline $21-J u l-15$ & Lewiston Bridge & Steel Bridge & $3^{\text {rd }}$ survey GRTS 4 to 53 \\
\hline
\end{tabular}




\section{Appendix B. U.S. Geological Survey Hygiene Protocol for Control of Disease Transmission Between Amphibian Study Sites}

\section{USGS JUNE2015}

To be completed between any sites that are not "water-connected", or that amphibians do not freely move between.

To be completed on all gear/equipment that may have touched site water or especially amphibians, including but not limited to:

- Waders

- Shoes/boots

- Dip nets

- Rulers and other instruments

- Specimen bags/containers

- Traps

\section{Materials:}

- Plastic bucket with handle for sterilization and holding cleaning gear

- Gallon of household chlorine bleach (6\% concentration of sodium hypochlorite)

- Two stiff scrub brushes with handles, one for sterilization, and one for cleaning off mud/dirt

- Rubber dishwashing gloves

- Spray bottle

\section{Procedure:}

1) Before leaving site, wash off in site water as much of the mud/dirt on equipment and gear and remove any vegetation or detritus attached to gear by shaking, rinsing in water and hand picking.

2) Do all sterilizing well away from streams or ponds.

3) Fill bucket with two gallons (7.6 liters) clear water (from pond, spigot or jug).

4) Add 1 cup (237 milliliters) of bleach (for a $\sim 4 \%$ concentration).

5) Stir to mix with brush.

6) Clean off any remaining vegetation or mud with stiff brush that may have been missed earlier.

7) Dip and rotate folded Minnow traps in solution, shake off, open and lay out in sun/wind to dry

8) Dip shoes in solution and scrub, shake off and let dry in sun.

9) Either dip and scrub waders in bucket or lay waders on ground and pour solution on them while scrubbing.

Spray bottle (with same solution concentration) can also be used to apply solution where needed.

10) Sterilize brushes in solution.

11) Any remaining sterilization solution can be saved in a sealable container for future use. If solution must be discarded, dispose of on asphalt, cement or hard roadbed, well away from any water bodies.

12) Allow all gear and equipment to dry completely before reuse at next site.

13) If complete drying after bleaching is not possible, gear and equipment can be washed with non-site water (or water from the next site to be visited) after a 15 minute wait, well away from the site. 


\section{For further reading:}

Interim Hygiene Protocols for Amphibian Field Staff and Researchers in Standard Operating Procedures: Hygiene Protocols for Amphibian Fieldwork, 2008. Ecosystems Branch, Ministry of Environment, British Columbia

Phillott and others, 2010, Minimizing exposure of amphibians to pathogens during field studies. Dis Aquat Organ. Nov; 92(2-3): 175-85.

NSW National Parks and Wildlife Service 2001. Hygiene protocol for the control of disease in frogs. Information Circular Number 6. NSW NPWS, Hurtsville NSW.

Berger and others, 2004, Hygiene protocol for handling amphibians in field studies

Threat Abatement Plan for Infection of amphibians with chytrid fungus resulting in chytridiomycosis 2004. Department of the Environmental and Heritage, Commonwealth of Australia (DRAFT) 


\section{Appendix C. Metadata for Database: Foothill Yellow-legged Frogs}

\section{RABO_GRTS}

We defined sample units on the main stem Trinity River corresponding to the generalized linear random tessellation stratified (GRTS) sample unit selection protocol defined by California Department of Fish and Game and others (2010). We survey all 5 panels that occur between the Steel Bridge river access and the confluence with the North Fork Trinity River each year. Individual tributaries were considered separate sample units. 15 of the 23 tributaries have been eliminated from future surveys due to either lack of breeding habitat, tributaries do not flow into the main stem, or permission was not granted from land owners.

1.1. ID: This is a unique identifier for the GRTS and Survey number and it used as the primary key linking the record to other tables. The first two digits are the last two digits of the survey year, the next 3 digits are the GRTS number (GRTS number is preceded by a zero if it is only a two-digit number). The number after the decimal point indicates the survey number. Hence, for example, survey two of GRTS 53 in 2014 would have the unique identifier of 14053.2

1.2. SurveyNum: Indicates the survey number $(1,2$, or 3$)$. This value is auto-filled based on the unique identifier ID.

1.3. GRTS: Number assigned to each GRTS sample unit as described in appendix E.

1.4. StartTime: Recorded as HR:MIN:SEC, indicates the time the GRTS survey was initiated. If the GRTS was not completed in one day, the time at the start of the first visit is recorded.

1.5. Trib Date: If there is a confluence with a surveyed tributary within the GRTS, record the date that this Tributary was surveyed.

1.6. GRTSStartDate: Date and time that the survey is started Format: DD/MM/YYYY.

1.7. GRTSEndDate: Date that the survey is completed. Format: DD/MM/YYYY/.

*Note: GRTS start and end dates will usually be the same, however some GRTS sample units will be split based on the put-in and take-out locations, hence the start and end dates to may vary.

1.8. SurveyYear: Four-digit number indicating the year of the survey. This value autofills based on the unique identifier ID.

1.9. WaterTemp: Water temperature in degrees Celsius at start of survey in the main channel. If the unit was surveyed over more than one day, record the mean water temperature of the visits.

1.10. AirTemp: Air temperature in degrees Celsius at start of survey in the main channel. If the unit was surveyed over more than one day, record the mean air temperature of the visits.

1.11. Wind: Check one box that best describes the predominant wind conditions.

1.11.1. $1=$ calm

1.11.2. 2 = light breeze

1.11.3. 3 = strong breeze

1.11.4. 4 = gusts.

1.12. Weather: Check one box that best describes the weather conditions at the start of the survey.

1.12.1. $1=$ clear/partly cloudy

1.12.2. $2=$ overcast

1.12.3. 3 = light rain/showers

1.12.4. $4=$ heavy rain 
1.13. Survey Crew: Full names of the people surveying the site on this visit.

1.14. GRTSNotes Add any notes related to survey conditions or other factors that could affect detectability of frogs or egg masses (e.g., evidence of recent water level changes since breeding, other factors affecting search efficiency, etc.).

\section{RABOSubHabitat}

When habitat is identified that appears appropriate for frogs and egg masses (see SOP \#3), surveyors will disembark from kayaks and conduct assessments on foot. We consider a distinct SubHabitat to be an area that is separated from other potential breeding areas by features that are not favored frog habitat (e.g., steep cut banks, deep fast water, etc.).

2.1. ID: This is the same unique identifier for the GRTS and Survey number as in the RABO_GRTS table. It may be repeated multiple times in this table if more than one subhabitat is detected within the GRTS.

2.2. SurveyNum: Indicates the survey number $(1,2$, or 3$)$. This value us auto-filled based on the unique identifier ID.

2.3. GRTS: Number assigned to each GRTS sample unit as described in appendix E.

2.4. SubHabID: Subhabitats on the main stem are labeled based on the order in which they are visited within the GRTS sample unit, appending the GRTS number with a letter. For example, in GRTS 81, the first subhabitat would be labeled 81A, the second 81B, etc. Tributaries are labeled with the GRTS that contains the confluence and the letter T.

2.5. SubHabDate: The date the subhabitat was surveyed, MM/DD/YYYY

2.6. LengthMC: Length of the surveyed shoreline associated with the main channel of the river, from the GPS track

2.7. LengthSC: Length of the surveyed shoreline associated with a side channel that is open to the main channel on both sides

2.8. LengthBWC: Length of the surveyed shoreline associated with a backwater that is connected to the main channel on one side (does not flow through)

2.9. LengthBWU: Length of the surveyed shoreline associated with a backwater that is unconnected to the main channel

2.10. RiverSide: $R=$ Right, $L=L e f t, M=$ island within the main channel, $T=$ tributary

2.11. DominantVeg: Indicate the dominant vegetation at the gravel bar. Choose from: Grasses, Shrub, Hardwood, None, Other (describe)

2.12. Canopy Cover: Estimate how much of the survey area has overhanging canopy.

2.13. Substrate: Choose best description of dominant substrate: sand/silt, pebble, cobble, boulder, bedrock, other.

2.14. Latitude: Record starting location in decimal degrees

2.15. Longitude: Record starting location in decimal degrees

2.16. WaterTemp: Record the water temperature of the main channel near the shore of the subhabitat in degrees Celsius.

2.17. WaterTemp2: If appropriate, record water temperatures of any backwaters or side channels likely to be different from the main channel

2.18. WaterTemp3: If appropriate, record water temperatures of any backwaters or side channels likely to be different from the main channel

2.19. WaterTempNotes: Identify what WaterTemps 2 and 3 are representing.

2.20. AirTemp: Record air temperature in degrees Celsius at start of survey.

2.21. TimeStart: Start time of the survey (24-hr clock). Format: 0830 (=8:30 AM).

2.22. TimeEnd: End time of the survey (24-hr clock). Format: 1610 (=4:10 PM). 
2.23. TotalTime: Amount of time between Time Start and Time End when surveying, expressed in total number of minutes (excluding time when off effort to record details about sightings or for capturing animals).

2.24. Wind: Check one box that best describes the predominant wind conditions.

2.24.1. 1 = calm

2.24.2. 2 = light breeze

2.24.3. 3 = strong breeze

2.24.4. 4 = gusts.

2.25. Weather: Check one box that best describes the weather conditions at the start of the survey.

2.25.1. $1=$ clear/partly cloudy

2.25.2. 2 = overcast

2.25.3. 3 = light rain/showers

2.25.4. 4 = heavy rain

2.26. TrackNames: Record track name from GPS. Create separate tracks for different habitat types within the same subhabitat unit.

2.27. PhotoTimes: Record times when photos were taken

2.28. SubHabNotes: Add any notes related to survey conditions or other factors that could affect detectability of egg masses (e.g., evidence of recent water level changes since breeding, other factors affecting search efficiency, etc.). Also note changes in weather conditions over course of survey not captured by Start and End fields described above.

\section{RABODetections}

Record information for any herpetological species observed

3.1. ID: This is the same unique identifier for the GRTS and Survey number as in the RABO_GRTS table. It may be repeated multiple times in this table if more than one subhabitat is detected within the GRTS.

3.2. SurveyNum: Indicates the survey number (1, 2, or 3$)$. This value us auto-filled based on the unique identifier ID.

3.3. GRTS: Number assigned to each GRTS sample unit as described in appendix E.

3.4. SubHabID: Subhabitats on the main stem are labeled based on the order in which they are visited within the GRTS sample unit, appending the GRTS number with a letter. For example, in GRTS 81, the first subhabitat would be labeled 81A, the second $81 \mathrm{~B}$, etc. Tributaries are labeled with the GRTS that contains the confluence and the letter T.

3.5. Species: Record the 4 letter code identifying the genus and species, here are the most commonly observed:

3.5.1. ACMA Actinemys marmorata (western pond turtle)

3.5.2. ANBO Anaxyrus boreas (western toad)

3.5.3. DITE Dicamptodon tenebrosus (coastal giant salamander)

3.5.4. LICA Lithobates catesbeianus (bullfrog)

3.5.5. PSRE Pseudacris regilla (chorus frog)

3.5.6. RABO Rana boylii (foothill yellow-legged frog)

3.5.7. TAGR Taricha granulosa (rough-skinned newt)

3.5.8. THAT Thamnophis atratus (aquatic garter snake)

3.5.9. THSI Thamnophis sirtalis (common garter snake)

3.5.10. THSP Thamnophis species (if the above two cannot be distinguished)

3.6. Lifestage: Egg, Larva, Juvenile, Adult, Unknown Juvenile or Adult, Unknown

3.7. Actual: Record number if this is an exact count of all species/stage observed

3.8. Estimate: Record number if the count is an estimate of all species/stage observed. 
3.9. Method: Record how the species was observed by selecting one of the following: Captured, Visual, Auditory, Dead

3.10. OnEffort: yes or no, was the species observed during the timed survey (yes), or before or after the timed survey was completed (no), for example while walking back to kayaks after survey.

3.11. Waypoint: Record the waypoint associated with the sighting

3.12. Latitude: Record the location of the waypoint

3.13. Longitude: Record the location of the waypoint

3.14. Photos: Record any times of photos

3.15. DetectionNotes: Add any notes related to species data

4. RABOOviposition An Oviposition Site is a location where either communal or single foothill yellow-legged frog egg masses are found. We consider egg masses that are within the diameter of a large mass of one another $(<20 \mathrm{~cm})$ to be within the same Oviposition site.

4.1. ID: This is the same unique identifier for the GRTS and Survey number as in the RABO_GRTS table. It may be repeated multiple times in this table if more than one subhabitat is detected within the GRTS.

4.2. SurveyNum: Indicates the survey number $(1,2$, or 3$)$. This value us auto-filled based on the unique identifier ID.

4.3. GRTS: Number assigned to each GRTS sample unit as described in appendix E.

4.4. SubHabID: Subhabitats on the main stem are labeled based on the order in which they are visited within the GRTS sample unit, appending the GRTS number with a letter. For example, in GRTS 81, the first subhabitat would be labeled 81A, the second 81B, etc. Tributaries are labeled with the GRTS that contains the confluence and the letter T.

4.5. Species: Same as above

4.6. OvipID: Identify oviposition sites by the subhabitat ID, a dash, and a sequential number. For example if 2 oviposition sites are found at subhabitat $81 \mathrm{~A}$, the first would be $81 \mathrm{~A}-1-1$ and the second would be $81 \mathrm{~A}-2$.

4.7. Waypoint: record the waypoint for the oviposition site

4.8. Latitude: Record the location of the waypoint

4.9. Longitude: Record the location of the waypoint

4.10. OvipNotes: Any notes related to the Oviposition Site.

\section{RABOEggMass}

This section records more detailed information about the egg masses found at each oviposition site

5.1. ID: This is the same unique identifier for the GRTS and Survey number as in the RABO_GRTS table. It may be repeated multiple times in this table if more than one subhabitat is detected within the GRTS.

5.2. SurveyNum: Indicates the survey number $(1,2$, or 3$)$. This value us auto-filled based on the unique identifier ID.

5.3. GRTS: Number assigned to each GRTS sample unit as described in appendix E.

5.4. SubHabID: Subhabitats on the main stem are labeled based on the order in which they are visited within the GRTS sample unit, appending the GRTS number with a letter. For example, in GRTS 81, the first subhabitat would be labeled 81A, the second 81B, etc. Tributaries are labeled with the GRTS that contains the confluence and the letter T.

5.5. OvipID: Record the OvipID for the eggmass, consistent with the Oviposition table.

5.6. MassID: Identify individual egg masses by the subhabitat ID, a dash, and a sequential letter. For example if 2 oviposition sites are found at subhabitat 81A, 81 A-1 (with 3 egg masses) and 
81A-2 (with 2 egg masses), the egg masses at $81 \mathrm{~A}-1$ would be $81 \mathrm{~A}-\mathrm{A}, 81 \mathrm{~A}-\mathrm{B}$, and $81 \mathrm{~A}-\mathrm{C}$; and the egg masses at $81 \mathrm{~A}-2$ would be $81 \mathrm{~A}-\mathrm{D}$ and $81 \mathrm{~A}-\mathrm{E}$.

5.7. DistShore: measure the distance, in $\mathrm{cm}$, from the center of the egg mass to the shore, perpendicular to the shoreline

5.8. MassDepth:Total depth, in $\mathrm{cm}$, of the water column next to the mass

5.9. Gosner: Score developmental stages of eggs in a single egg mass using the attached table from Gosner (1960).

5.10. PCTdead: An estimate of the percentage of the egg mass, if any, that appears dead to the nearest 5\%. Dead embryos often, but not always, appear white. Fungal hyphae may be visible.

5.11. DepthBottom: Depth, in cm, from substrate to bottom of mass

5.12. DepthTop: Depth, in cm, from substrate to top of mass

5.13. Substrate: Select from Vegetation, pebble, cobble, boulder

5.14. WaterTemp: Record the water temperature, in degrees Celsius, near the egg mass

5.15. Photos: Record any times of photos

5.16. MassNotes: Record any details about the egg mass not covered by the fields listed, etc. Note whether an egg mass appears to have been blown or drifted from its oviposition site; this can be discernible if egg masses are in accumulations of flotsam. 


\section{Appendix D. Metadata for Database: Western Pond Turtles}

\section{Western pond turtle Survey}

The Survey Area is the entire study area, including $62 \mathrm{~km}$ of the main stem Trinity River and $400 \mathrm{~m}$ up each of the 23 major tributaries found along this stretch.

1.1. SurveyID: Number of survey, 1, 2, or 3.

1.2. DateStart: Day, month and year on which survey is initiated. Format: MM/DD/YYYY.

1.3. DateEnd: Day, month and year on which survey is completed. Format: MM/DD/YYYY.

1.4. SurveyNotes: Enter any note specific to the survey.

\section{Western Pond TurtleUnit}

Each unit on the main stem is a 400-m segment, starting with unit 4 at the Lewiston Weir and ending with unit 159 at the confluence with the North Fork Trinity River. Each pond is an individual unit.

2.1. Survey: Survey number for the unit, 1, 2, or 3 .

2.2. UnitID: GRTS number 4 through 159 or the name of the pond.

2.3. UnitType: Choose from:

2.3.1. MC: main channel

2.3.2. Pond

2.3.3. BWC: Back water off the main channel but that is connected to the main channel (i.e. likely to have different temperature and flow characteristics)

2.3.4. BWU: Backwater immediately adjacent to the main channel, likely connected at high water levels but is unconnected at the time of the survey.

2.4. StartDate: Enter the date and time that the survey is started Format:

$\mathrm{DD} / \mathrm{MM} / \mathrm{YYYY} / \mathrm{HR} / \mathrm{MIN} / \mathrm{SEC}$.

2.5. EndDate: Enter the date that the survey is completed. Format: DD/MM/YYYY/HR/MIN/SEC. $* * *$ NOTE*** Reach start and end date will almost always be the same day. The exceptions are at the put-ins and take-outs, which usually occur somewhere in the middle of a unit. In this case only a portion of the unit will be completed on a given day, with the remainder completed when the put-in/take-out schedule incorporates the remainder of the unit.

2.6. Survey Crew: Full names of the people surveying the site on this visit.

2.7. StartTime: Time at the beginning of the unit

2.8. EndTime: Time at the beginning of the next unit

2.9. TotalTime: Total time to float unit, excluding breaks and off effort times to catch up on data entry/recording

2.10. WaterTemp: Record water temperature in degrees Celsius at start of survey in the main channel

2.11. AirTemp: Record air temperature in degrees Celsius at start of survey in the main channel Wind: Check one box that best describes the wind conditions at start of the survey: calm, light breeze, strong breeze, gusts.

2.12. Wind: Check one box that best describes the predominant wind conditions.

2.12.1. $1=$ calm

2.12.2. 2 = light breeze

2.12.3. 3 = strong breeze

2.12.4. $4=$ gusts.

2.13. Weather: Check one box that best describes the weather conditions at the start of the survey.

2.13.1. $1=$ clear/partly cloudy

2.13.2. 2 = overcast 
2.13.3. 3 = light rain/showers

2.13.4. 4 = heavy rain

2.14. TurtleUnitNotes Add any notes related to survey conditions or other factors that could affect detectability of western pond turtles.

3. General Habitat

Characterizes the predominant habitat characteristics for each unit

3.1. UnitID: GRTS number 4 through 159 or the name of the pond.

3.2. HabDate: Record the date that the habitat was assessed, MM/DD/YYYY

3.3. Side Indicate the river side if the unit is on the main channel, or the unit type if the unit is not on the main channel, choose from:

3.3.1. River right

3.3.2. River left

3.3.3. Pond

3.3.4. BWC: Back water off the main channel but that is connected to the main channel (i.e. likely to have different temperature and flow characteristics)

3.3.5. BWU: Backwater immediately adjacent to the main channel, likely connected at high water levels but is unconnected at the time of the survey.

3.4. Vegetation: Choose from

3.4.1. Unvegetated, at least $2 / 3$ of shoreline is gravel

3.4.2. Open vegetation, grass

3.4.3. Immature, $2 / 3$ willow

3.4.4. Mixed willow and hardwood

3.4.5. Mature, at least $2 / 3$ hardwood

3.5. Bank: Choose from

3.5.1. Cut bank with exposed soil

3.5.2. Cut bank, stabilized with tree roots

3.5.3. Sloped unvegetated bank

3.5.4. Sloped vegetated bank

3.6. Basking: Choose from

3.6.1. No basking structures

3.6.2. Few basking structures (less than 5 per $50 \mathrm{~m}$ )

3.6.3. Moderate basking structures ( 5 to 15 per $50 \mathrm{~m}$ )

3.6.4. High basking structures (greater than 15 per $50 \mathrm{~m}$ )

3.7. Velocity: Choose from

3.7.1. Flat water along bank and under basking structures

3.7.2. Moving water along bank and under basking structures

3.7.3. White water along bank

3.8. Depth: Choose from

3.8.1. Shallow, less than $10 \mathrm{~cm}$, under basking habitat

3.8.2. $10-100 \mathrm{~cm}$ under basking habitat

3.8.3. Deep, greater than $100 \mathrm{~cm}$, under basking habitat

3.9. GeneralHabitatNotes: Any notes related to the basking Site.

4. TurtleDetections

Complete when basking western pond turtles are sighted

4.1. Survey: Survey number for the unit, 1, 2, or 3 .

4.2. UnitID: GRTS number 4 through 159 or the name of the pond.

4.3. UnitType: Choose from: 
4.3.1. MC: main channel

4.3.2. Pond

4.3.3. BWC: Back water off the main channel but that is connected to the main channel (i.e. likely to have different temperature and flow characteristics)

4.3.4. BWU: Backwater immediately adjacent to the main channel, likely connected at high water levels but is unconnected at the time of the survey.

4.4. SightingID: record the unique waypoint for the basking site

4.5. Latitude: Record the location of the basking site

4.6. Longitude: Record the location of the basking site

4.7. Lifestage: Choose from

4.7.1. Hatchling, juvenile, adult, unknown juvenile/adult

***NOTE*** sightings will most often be classified as unknown juvenile/adult

4.8. Actual: Record number if this is an exact count of all species/stage observed

4.9. Estimate: Record number if the count is an estimate of all species/stage observed.

4.10. Method: Record how the species was observed by selecting one of the following:

Captured, visual, auditory, dead

4.11. OnEffort: yes or no, was the species observed during the timed survey (yes), or before or after the timed survey was completed (no), for example while walking back to kayaks after survey.

4.12. RiverSide: Choose from:

4.12.1. RR River Right

4.12.2. RL River Left

4.12.3. SR Side Channel Right (if the main channel is split by and island)

4.12.4. SL Side Channel Left

4.12.5. Pond

4.12.6. BWC: Back water off the main channel but that is connected to the main channel (i.e. likely to have different temperature and flow characteristics)

4.12.7. BWU: Backwater immediately adjacent to the main channel, likely connected at high water levels but is unconnected at the time of the survey.

4.13. Behavior: Choose from

4.13.1. B_log: Basking on log

4.13.2. B_rock: Basking on rock

4.13.3. B_shore: Basking on shore

4.13.4. B_water: Basking in water (only snout visible)

4.14. Vegetation: Choose from

4.14.1. Unvegetated, at least $2 / 3$ of shoreline is gravel

4.14.2. Open vegetation, grass

4.14.3. Immature, $2 / 3$ willow

4.14.4. Mixed willow and hardwood

4.14.5. Mature, at least $2 / 3$ hardwood

4.15. Bank: Choose from

4.15.1. Cut bank with exposed soil

4.15.2. Cut bank, stabilized with tree roots

4.15.3. Sloped unvegetated bank

4.15.4. Sloped vegetated bank

4.16. Basking: Choose from

4.16.1. No basking structures

4.16.2. Few basking structures (less than 5 per $50 \mathrm{~m}$ ) 
4.16.3. Moderate basking structures ( 5 to 15 per $50 \mathrm{~m}$ )

4.16.4. High basking structures (greater than 15 per $50 \mathrm{~m}$ )

4.17. Velocity: Choose from

4.17.1. Flat water along bank and under basking structures

4.17.2. Moving water along bank and under basking structures

4.17.3. White water along bank

4.18. Depth: Choose from

4.18.1. Shallow, less than $10 \mathrm{~cm}$, under basking habitat

4.18.2. $10-100 \mathrm{~cm}$ under basking habitat

4.18.3. Deep, greater than $100 \mathrm{~cm}$, under basking habitat

4.19. TurtleDetectionNotes: Any notes related to the basking Site.

\section{OtherDetections}

Record information for any herpetological species other than western pond turtles that are observed 5.1. Survey: Survey number for the unit, 1, 2, or 3.

5.2. TurtleUnitID: GRTS number 4 through 159 or the name of the pond.

5.3. Species: Record the 4 letter code identifying the genus and species:

5.3.1. ANBO Anaxyrus boreas (western toad)

5.3.2. DITE Dicamptodon tenebrosus (coastal giant salamander)

5.3.3. LICA Lithobates catesbeianus (bullfrog)

5.3.4. PSRE Pseudacris regilla (chorus frog)

5.3.5. RABO Rana boylii (foothill yellow-legged frog)

5.3.6. SCOC Sceloporus occidentalis (western fence lizard)

5.3.7. TAGR Taricha granulosa (rough-skinned newt)

5.3.8. THAT Thamnophis atratus (aquatic garter snake)

5.3.9. THSI Thamnophis sirtalis (common garter snake)

5.3.10. THSP Thamnopis species (if the above two cannot be distinguished)

5.3.11. EUSK Eumeces skiltonianus (western skink)

5.4. Lifestage: Egg, Larva, Juvenile, Adult, Unknown Juvenile or Adult, Unknown

5.5. Actual: Record number if this is an exact count of all species/stage observed

5.6. Estimate: Record number if the count is an estimate of all species/stage observed.

5.7. Method: Record how the species was observed by selecting one of the following:

5.7.1. Captured

5.7.2. Visual

5.7.3. Auditory

5.7.4. Dead

5.8. OnEffort: yes or no, was the species observed during the timed survey (yes), or before or after the timed survey was completed (no), for example while walking back to kayaks after survey.

5.9. SpeciesNotes: Add any notes related to species data 


\section{Appendix E. Generalized Linear Random Tessellation Stratified Sampling Protocol (GRTS) Panel Identification}

[GRTS Sample Unit values are the unit numbers used in the fish studies, and GRTS Panel indicates which panel each units belongs in, and hence which years they are sampled in the fish studies (California Department of Fish and Game and others, 2010). GRTSID is the value we use as these are sequential along the river, with lower values the closest to the Lewiston Dam]

\begin{tabular}{|c|c|c|c|c|c|}
\hline GRTSID & GRTS Sample Unit & GRTS Panel & GRTSID & GRTS Sample Unit & GRTS Panel \\
\hline 7 & GRTS400-30 & panel_2 & 83 & GRTS400-16 & panel_1 \\
\hline 8 & GRTS400-67 & panel_5 & 86 & GRTS400-56 & panel_4 \\
\hline 9 & GRTS400-35 & panel_3 & 88 & GRTS400-32 & panel_2 \\
\hline 11 & GRTS400-51 & panel 4 & 91 & GRTS400-64 & panel 4 \\
\hline 13 & GRTS400-78 & panel_5 & 92 & GRTS400-80 & panel_5 \\
\hline 14 & GRTS400-46 & panel_3 & 93 & GRTS400-48 & panel_3 \\
\hline 15 & GRTS400-42 & panel_3 & 96 & GRTS400-70 & panel_5 \\
\hline 17 & GRTS400-14 & panel_1 & 97 & GRTS400-22 & panel_2 \\
\hline 18 & GRTS400-58 & panel_4 & 98 & GRTS400-54 & panel_4 \\
\hline 21 & GRTS400-03 & panel_1 & 100 & GRTS400-38 & panel_3 \\
\hline 24 & GRTS400-19 & panel_2 & 104 & GRTS400-50 & panel_4 \\
\hline 27 & GRTS400-71 & panel_5 & 106 & GRTS400-06 & panel_1 \\
\hline 29 & GRTS400-23 & panel_2 & 110 & GRTS400-74 & panel_5 \\
\hline 30 & GRTS400-07 & panel_1 & 112 & GRTS400-26 & panel_2 \\
\hline 32 & GRTS400-39 & panel_3 & 113 & GRTS400-10 & panel_1 \\
\hline 34 & GRTS400-43 & panel_3 & 116 & GRTS400-25 & panel_2 \\
\hline 36 & GRTS400-11 & panel_1 & 118 & GRTS400-09 & panel_1 \\
\hline 38 & GRTS400-75 & panel_5 & 121 & GRTS400-29 & panel_2 \\
\hline 40 & GRTS400-27 & panel_2 & 122 & GRTS400-13 & panel_1 \\
\hline 42 & GRTS400-55 & panel_4 & 123 & GRTS400-73 & panel_5 \\
\hline 46 & GRTS400-79 & panel_5 & 125 & GRTS400-41 & panel_3 \\
\hline 47 & GRTS400-31 & panel_2 & 126 & GRTS400-57 & panel_4 \\
\hline 50 & GRTS400-47 & panel_3 & 131 & GRTS400-45 & panel_3 \\
\hline 51 & GRTS400-63 & panel_4 & 132 & GRTS400-77 & panel_5 \\
\hline 52 & GRTS400-59 & panel_4 & 133 & GRTS400-02 & panel_1 \\
\hline 55 & GRTS400-15 & panel_1 & 134 & GRTS400-61 & panel_4 \\
\hline 58 & GRTS400-20 & panel_2 & 138 & GRTS400-18 & panel_2 \\
\hline 60 & GRTS400-68 & panel_5 & 140 & GRTS400-66 & panel_5 \\
\hline 61 & GRTS400-04 & panel_1 & 141 & GRTS400-53 & panel_4 \\
\hline 62 & GRTS400-36 & panel_3 & 142 & GRTS400-34 & panel_3 \\
\hline 66 & GRTS400-52 & panel_4 & 145 & GRTS400-21 & panel_2 \\
\hline 67 & GRTS400-24 & panel_2 & 148 & GRTS400-05 & panel_1 \\
\hline 68 & GRTS400-72 & panel_5 & 149 & GRTS400-17 & panel_2 \\
\hline 71 & GRTS400-08 & panel_1 & 151 & GRTS400-65 & panel_5 \\
\hline 72 & GRTS400-28 & panel_2 & 152 & GRTS400-01 & panel_1 \\
\hline 74 & GRTS400-76 & panel_5 & 155 & GRTS400-33 & panel_3 \\
\hline 75 & GRTS400-60 & panel_4 & 156 & GRTS400-49 & panel_4 \\
\hline 78 & GRTS400-40 & panel_3 & 157 & GRTS400-37 & panel_3 \\
\hline 80 & GRTS400-44 & panel_3 & 158 & GRTS400-69 & panel_5 \\
\hline 81 & GRTS400-12 & panel_1 & & & \\
\hline
\end{tabular}




\section{Appendix F. Field Datasheet: Foothill Yellow-legged} Frogs

RABO SURVEYS - SUBHABITAT DATA

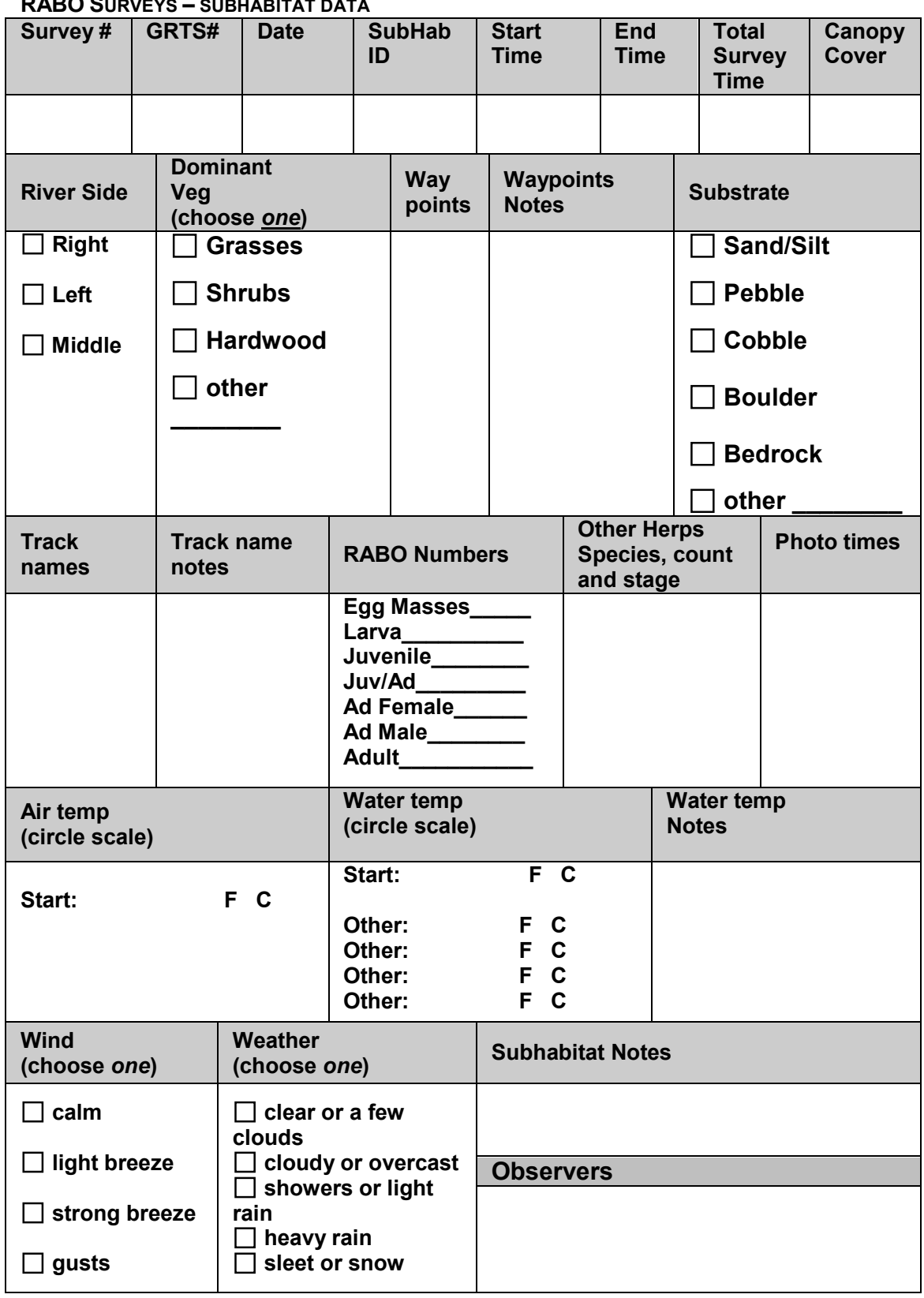

RABO SURVEYS -DETECTIONS

\begin{tabular}{|c|c|c|c|c|c|}
\hline \multirow{2}{*}{$\begin{array}{l}\text { Observers } \\
\text { Species } \\
\text { Code }\end{array}$} & & & \multicolumn{2}{|c|}{ Date } & \multirow{2}{*}{ 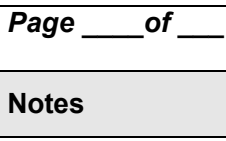 } \\
\hline & Stage & Count & $\begin{array}{l}\text { Photo } \\
\text { times }\end{array}$ & SubHabitat ID & \\
\hline & & A & & & \\
\hline & & E & & & \\
\hline & & A & & & \\
\hline & & $E$ & & & \\
\hline & & A & & & \\
\hline & & $E$ & & & \\
\hline & & A & & & \\
\hline & & $E$ & & & \\
\hline & & A & & & \\
\hline & & E & & & \\
\hline & & A & & & \\
\hline & & E & & & \\
\hline & & A & & & \\
\hline & & E & & & \\
\hline
\end{tabular}

Stage: Egg/mass (EM); Hatchling (H); Larvae (L); Metamorph (M); Paedomorph (P); Juvenile (J); Adult (A), Juvenile/Adult (J/A), Adult Male (M) Adult Female (F)

Amphibians Reptiles Crayfish

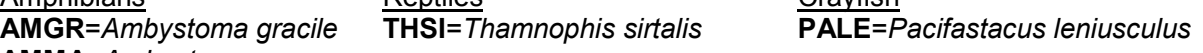
AMMA=Ambystoma macrodactylum ANBO=Anaxyrus boreas DITE=Dicamptodon THEL=Thamonophis elegans THSP=Thamonophis species ACMA=Actinemys marmorata PRCL=Procambarus clarkii tenebrosus LICA=Lithobates catesbeiananus PSRE=Pseudacris regilla RABO=Rana boylii RACA=Rana cascadae RAPR=Rana pretiosa TAGR=Taricha granulosa
LAGE=Lampropeltis getula
Denectes neglectus

ORRU=Orconectes rusticus 


\section{RABO Monitoring Oviposition \& Eggmass Data}

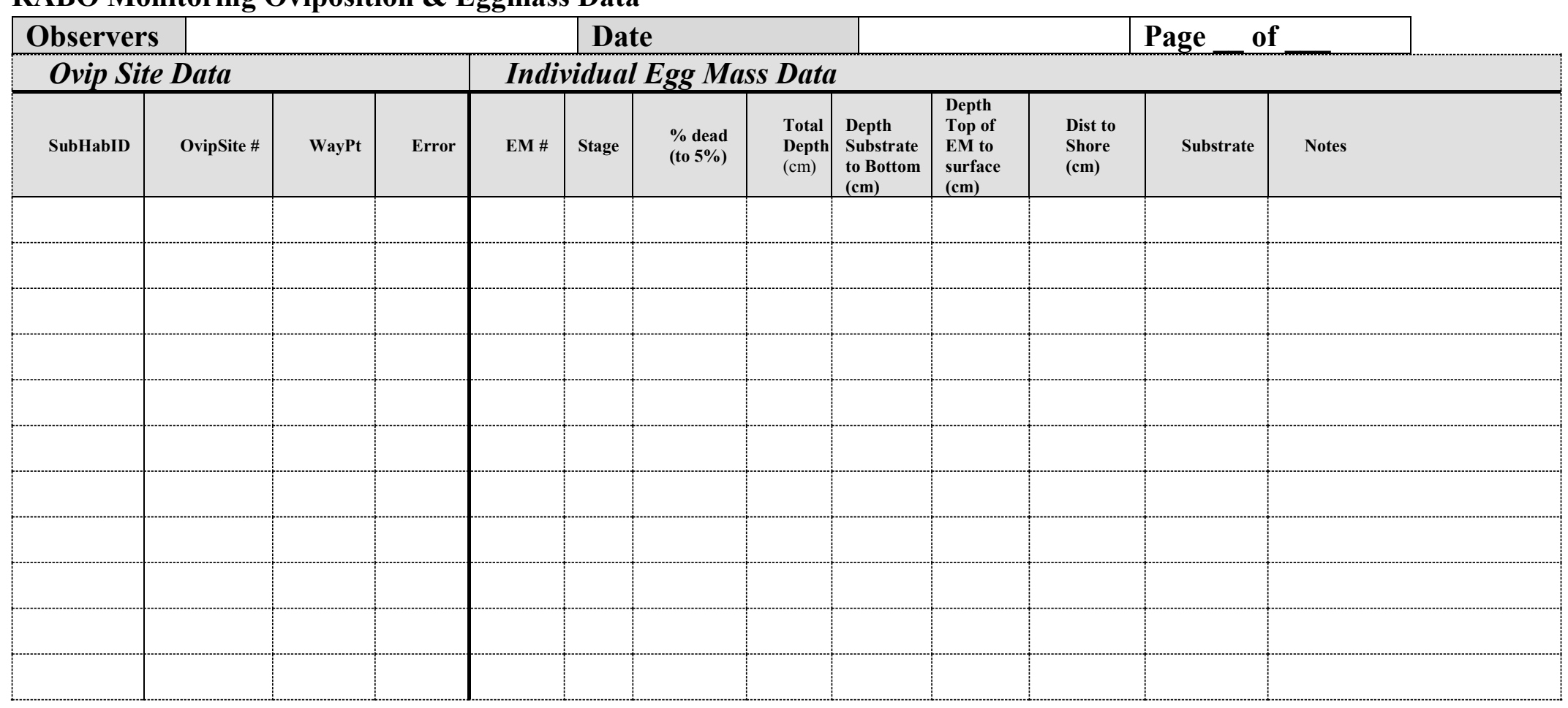


ACMA Incidental Capture Data

Observers

Date

Page

of

\begin{tabular}{|l|l|l|l|l|l|}
\hline SubHab ID & & & & \\
\hline Way Pt & & & & \\
\hline Photo Times & & & & \\
\hline Max SCL (mm) & & & & \\
\hline Min SCL (mm) & & & & & \\
\hline SCW (mm) & & & & & \\
\hline Depth (mm) & & & & & \\
\hline Max SPL (mm) & & & & & \\
\hline Min SPL (mm) & & & & & \\
\hline SPW (mm) & & & & & \\
\hline Mass (g) & & & & & \\
\hline Sex & & & & \\
\hline Age & & & & & \\
\hline Gravid & & & & \\
\hline Mark & & & & \\
\hline TempMark & & & & \\
\hline Notes & & & & & \\
\hline
\end{tabular}




\section{Appendix G. Field Datasheets-Western Pond Turtles}

Datasheet 1: record general habitat characteristics for each unit, no surveys for western pond turtles conducted

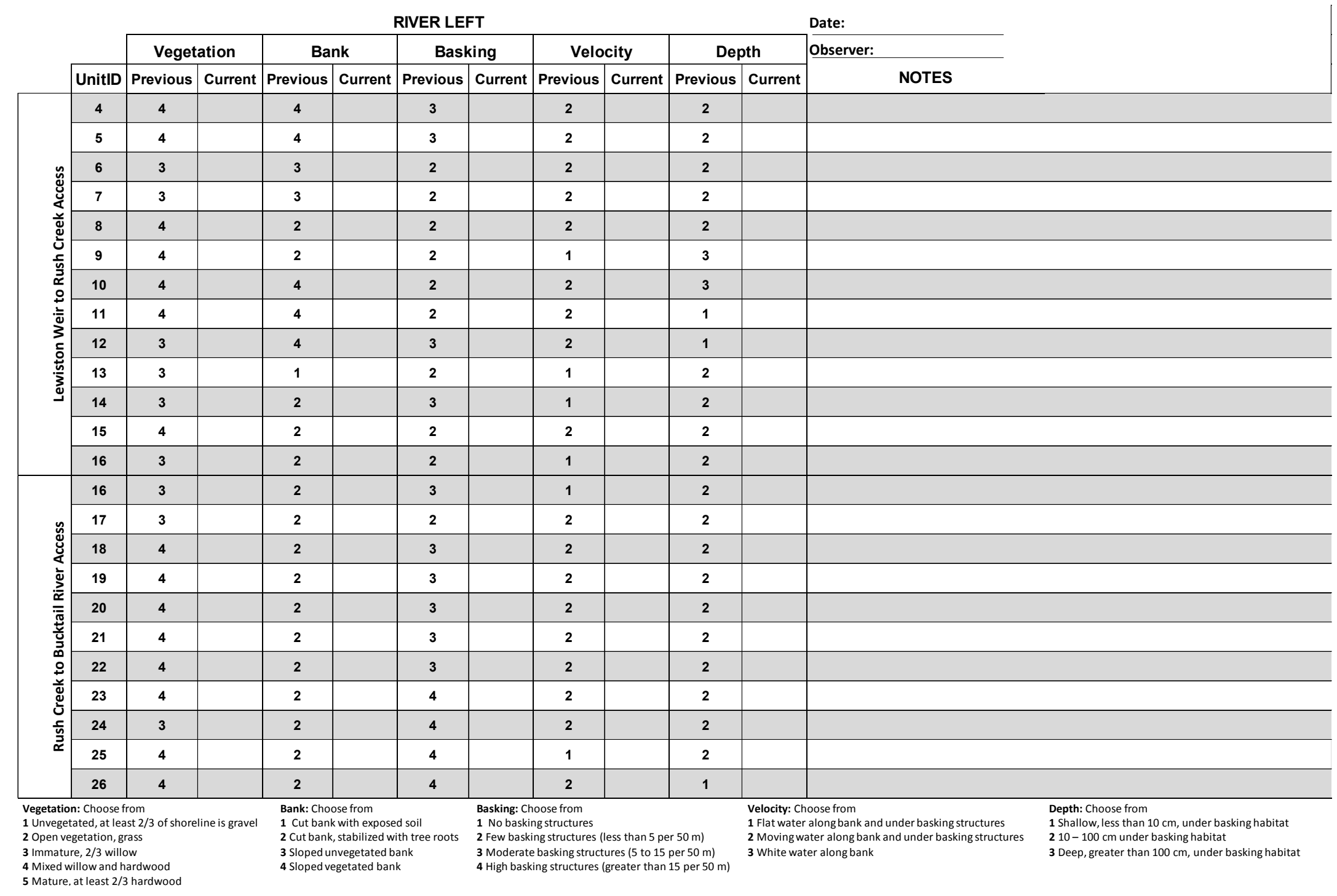




\begin{tabular}{|c|c|c|c|c|c|c|c|c|c|c|c|c|c|}
\hline & \multirow[b]{3}{*}{ UnitID } & \multicolumn{10}{|c|}{ RIVER LEFT } & Date: & \\
\hline & & \multicolumn{2}{|c|}{ Vegetation } & \multicolumn{2}{|c|}{ Bank } & \multicolumn{2}{|c|}{ Basking } & \multicolumn{2}{|c|}{ Velocity } & \multicolumn{2}{|c|}{ Depth } & \multirow{2}{*}{ Observer: } & \\
\hline & & Previous & Current & Previous & Current & \begin{tabular}{|l|} 
Previous \\
\end{tabular} & Current & Previous & Current & \begin{tabular}{|l|} 
Previous \\
\end{tabular} & Current & & \\
\hline \multirow{28}{*}{ 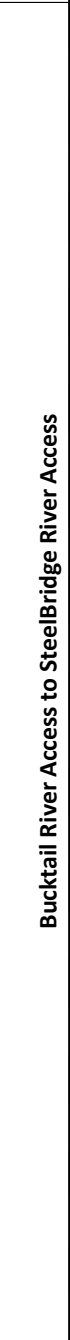 } & 26 & 4 & & 2 & & 4 & & 1 & & 2 & & & \\
\hline & 27 & 4 & & 2 & & 2 & & 2 & & 2 & & & \\
\hline & 28 & 2 & & 4 & & 2 & & 2 & & 2 & & & \\
\hline & 29 & 3 & & 4 & & 4 & & 1 & & 2 & & & \\
\hline & 30 & 3 & & 2 & & 2 & & 2 & & 2 & & & \\
\hline & 31 & 3 & & 3 & & 2 & & 2 & & 2 & & & \\
\hline & 32 & 3 & & 4 & & 2 & & 2 & & 2 & & & \\
\hline & 33 & 1 & & 3 & & 2 & & 1 & & 2 & & & \\
\hline & 34 & 4 & & 2 & & 3 & & 2 & & 2 & & & \\
\hline & 35 & 4 & & 2 & & 3 & & 2 & & 2 & & & \\
\hline & 36 & 4 & & 2 & & 3 & & 2 & & 2 & & & \\
\hline & 37 & 4 & & 2 & & 4 & & 2 & & 2 & & & \\
\hline & 38 & 4 & & 2 & & 3 & & 2 & & 2 & & & \\
\hline & 39 & 4 & & 2 & & 2 & & 2 & & 2 & & & \\
\hline & 40 & 4 & & 2 & & 3 & & 2 & & 2 & & & \\
\hline & 41 & 4 & & 2 & & 4 & & 2 & & 3 & & & \\
\hline & 42 & 2 & & 3 & & 2 & & 2 & & 2 & & & \\
\hline & 43 & 2 & & 4 & & 2 & & 2 & & 1 & & & \\
\hline & 44 & 2 & & 4 & & 2 & & 2 & & 2 & & & \\
\hline & 45 & 4 & & 2 & & 2 & & 2 & & 3 & & & \\
\hline & 46 & 4 & & 2 & & 2 & & 2 & & 2 & & & \\
\hline & 47 & 3 & & 4 & & 2 & & 2 & & 2 & & & \\
\hline & 48 & 4 & & 2 & & 3 & & 2 & & 2 & & & \\
\hline & 49 & 4 & & 2 & & 3 & & 2 & & 2 & & & \\
\hline & 50 & 4 & & 2 & & 3 & & 2 & & 2 & & & \\
\hline & 51 & 4 & & 2 & & 3 & & 2 & & 2 & & & \\
\hline & 52 & 4 & & 2 & & 3 & & 2 & & 2 & & & \\
\hline & 53 & 3 & & 3 & & 2 & & 2 & & 1 & & & \\
\hline 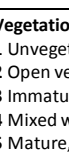 & 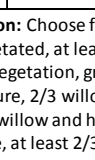 & $\begin{array}{l}\text { rom } \\
\text { st } 2 / 3 \text { of shore } \\
\text { rass } \\
\text { ow } \\
\text { ardwood } \\
\text { a hardwood }\end{array}$ & eline is gravel & $\begin{array}{l}\text { Bank: Cho } \\
1 \text { Cut bank } \\
2 \text { Cut bank } \\
3 \text { slopedu } \\
4 \text { sloped ve }\end{array}$ & 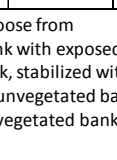 & $\begin{array}{l}\text { ed soil } \\
\text { ithtree roots } \\
\text { ank } \\
\text { kk }\end{array}$ & $\begin{array}{l}\text { Basking: ch } \\
\text { 1 N o baski } \\
2 \text { Few wask } \\
3 \text { Moderate } \\
4 \text { High bask }\end{array}$ & $\begin{array}{l}\text { loose from } \\
\text { ng structures } \\
\text { ing structures } \\
\text { bakking structc } \\
\text { ing structures }\end{array}$ & 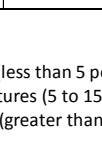 & 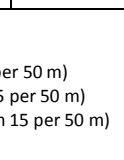 & $\begin{array}{l}\text { Velocity: Ch } \\
1 \text { Flatwater } \\
2 \text { Moving wa } \\
3 \text { White wate }\end{array}$ & $\begin{array}{l}\text { hoose from } \\
\text { ralongbank and under basking structures } \\
\text { vater along bank and under basking structures } \\
\text { ter along bank }\end{array}$ & 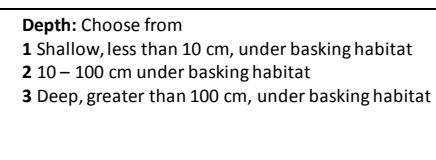 \\
\hline
\end{tabular}




\begin{tabular}{|c|c|c|c|c|c|c|c|c|c|c|c|c|c|}
\hline & \multirow[b]{3}{*}{ UnitID } & \multicolumn{10}{|c|}{ RIVER LEFT } & Date: & \\
\hline & & \multicolumn{2}{|c|}{ Vegetation } & \multicolumn{2}{|c|}{ Bank } & \multicolumn{2}{|c|}{ Basking } & \multicolumn{2}{|c|}{ Velocity } & \multicolumn{2}{|c|}{ Depth } & \multirow{2}{*}{ Observer: } & \\
\hline & & Previous & Current & \begin{tabular}{|l|} 
Previous \\
\end{tabular} & Current & Previous & Current & Previous & Current & Previous & Current & & \\
\hline \multirow{15}{*}{ 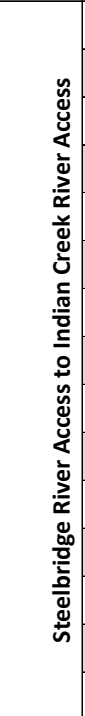 } & 53 & 3 & & 3 & & 2 & & 2 & & 1 & & & \\
\hline & 54 & 4 & & 2 & & 4 & & 2 & & 2 & & & \\
\hline & 55 & 4 & & 4 & & 2 & & 2 & & 2 & & & \\
\hline & 56 & 4 & & 4 & & 3 & & 2 & & 3 & & & \\
\hline & 57 & 4 & & 4 & & 3 & & 2 & & 2 & & & \\
\hline & 58 & 4 & & 2 & & 4 & & 2 & & 2 & & & \\
\hline & 59 & 4 & & 2 & & 4 & & 2 & & 2 & & & \\
\hline & 60 & 4 & & 2 & & 3 & & 2 & & 2 & & & \\
\hline & 61 & 2 & & 4 & & 2 & & 2 & & 2 & & & \\
\hline & 62 & 4 & & 2 & & 3 & & 2 & & 3 & & & \\
\hline & 63 & 4 & & 2 & & 3 & & 1 & & 3 & & & \\
\hline & 64 & 4 & & 2 & & 3 & & 1 & & 3 & & & \\
\hline & 65 & 4 & & 2 & & 3 & & 2 & & 2 & & & \\
\hline & 66 & 2 & & 4 & & 2 & & 1 & & 3 & & & \\
\hline & 67 & 4 & & 2 & & 3 & & 2 & & 2 & & & \\
\hline \multirow{13}{*}{ 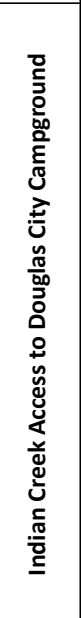 } & 67 & 4 & & 2 & & 3 & & 2 & & 2 & & & \\
\hline & 68 & 4 & & 2 & & 3 & & 2 & & 1 & & & \\
\hline & 69 & 1 & & 3 & & 1 & & 2 & & 2 & & & \\
\hline & 70 & 2 & & 3 & & 2 & & 2 & & 2 & & & \\
\hline & 71 & 4 & & 4 & & 3 & & 2 & & 2 & & & \\
\hline & 72 & 4 & & 2 & & 3 & & 2 & & 2 & & & \\
\hline & 73 & 4 & & 2 & & 3 & & 2 & & 2 & & & \\
\hline & 74 & 4 & & 2 & & 3 & & 2 & & 2 & & & \\
\hline & 75 & 4 & & 2 & & 2 & & 2 & & 2 & & & \\
\hline & 76 & 4 & & 2 & & 4 & & 1 & & 2 & & & \\
\hline & 77 & 4 & & 2 & & 3 & & 2 & & 2 & & & \\
\hline & 78 & 4 & & 2 & & 3 & & 2 & & 3 & & & \\
\hline & 79 & 4 & & 2 & & 4 & & 2 & & 2 & & & \\
\hline \multicolumn{4}{|c|}{$\begin{array}{l}\text { Vegetation: Choose from } \\
1 \text { Unvegetated, at least } 2 / 3 \text { of shoreline is gravel } \\
2 \text { Open vegetataio, }, \text { rass } \\
3 \text { Imature, } / 2 \text { willow } \\
4 \text { Mixed willow wand hardwood } \\
5 \text { Mature, at least } 2 / 3 \text { hardwood }\end{array}$} & $\begin{array}{l}\text { Bank: choc } \\
\text { 1 cutbank } \\
2 \text { Cutbank } \\
3 \text { Stlopedu } \\
4 \text { sloped ve }\end{array}$ & $\begin{array}{l}\text { oose from } \\
\text { nkwith expose } \\
k \text {, stabilized wi } \\
\text { unvegetated ba } \\
\text { vegetated bank }\end{array}$ & $\begin{array}{l}\text { d soil } \\
\text { ith tree roots } \\
\text { ank } \\
\text { an }\end{array}$ & $\begin{array}{l}\text { Basking: CK } \\
1 \text { No baski } \\
2 \text { 2 Few bask } \\
3 \text { Moderat } \\
4 \text { High bash }\end{array}$ & 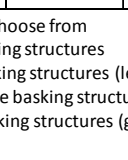 & $\begin{array}{l}\text { lless than } 5 \text { pe } \\
\text { tures } 5 \text { to } 15 \\
\text { (greater than }\end{array}$ & $\begin{array}{l}\text { eer } 50 \mathrm{~m} \text { ) } \\
\text { per } 50 \mathrm{~m} \text { ) } \\
\text { n } 15 \text { per } 50 \mathrm{~m} \text { ) }\end{array}$ & \multicolumn{2}{|c|}{$\begin{array}{l}\text { Velocity: Choose from } \\
1 \text { Flat water alonganank and under basking structures } \\
2 \text { Moving water along bank and under basking structures } \\
3 \text { White water along bank }\end{array}$} & $\begin{array}{l}\text { Depth: Choose from } \\
1 \text { Shallow, less than } 10 \mathrm{~cm} \text {, under basking habitat } \\
2100-10 \mathrm{~cm} \text { under basking habitat } \\
3 \text { Deee, greater than } 100 \mathrm{~cm} \text {, under basking habitat }\end{array}$ \\
\hline
\end{tabular}


RIVER LEFT

Date:

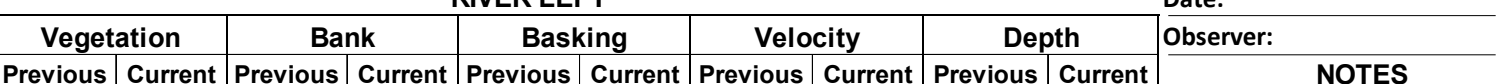

\begin{tabular}{|c|c|c|c|c|c|c|c|c|c|c|c|c|c|}
\hline \multirow{15}{*}{ 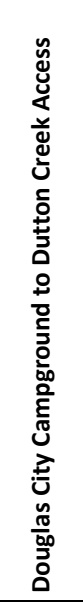 } & UnitID & Previous & Current & Previous & Current & Previous & Current & Previous & Current & Previous & Current & NOIES & \\
\hline & 80 & 4 & & 2 & & 3 & & 2 & & 2 & & & \\
\hline & 81 & 4 & & 2 & & 3 & & 2 & & 2 & & & \\
\hline & 82 & 4 & & 2 & & 2 & & 2 & & 3 & & & \\
\hline & 83 & 4 & & 2 & & 3 & & 2 & & 2 & & & \\
\hline & 84 & 4 & & 2 & & 2 & & 2 & & 2 & & & \\
\hline & 85 & 4 & & 2 & & 4 & & 2 & & 2 & & & \\
\hline & 86 & 4 & & 2 & & 2 & & 2 & & 3 & & & \\
\hline & 87 & 4 & & 2 & & 3 & & 2 & & 3 & & & \\
\hline & 88 & 4 & & 2 & & 3 & & 2 & & 3 & & & \\
\hline & 89 & 4 & & 2 & & 2 & & 2 & & 2 & & & \\
\hline & 90 & 4 & & 2 & & 3 & & 2 & & 2 & & & \\
\hline & 91 & 3 & & 2 & & 3 & & 2 & & 1 & & & \\
\hline & 92 & 4 & & 2 & & 4 & & 2 & & 2 & & & \\
\hline & 93 & 4 & & 2 & & 3 & & 2 & & 2 & & & \\
\hline \multirow{19}{*}{ 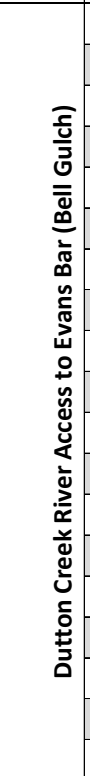 } & 93 & 4 & & 2 & & 3 & & 2 & & 2 & & & \\
\hline & 94 & 4 & & 2 & & 4 & & 2 & & 2 & & & \\
\hline & 95 & 4 & & 2 & & 3 & & 2 & & 3 & & & \\
\hline & 96 & 4 & & 2 & & 4 & & 1 & & 3 & & & \\
\hline & 97 & 4 & & 2 & & 3 & & 2 & & 3 & & & \\
\hline & 98 & 4 & & 2 & & 3 & & 2 & & 3 & & & \\
\hline & 99 & 4 & & 2 & & 3 & & 2 & & 3 & & & \\
\hline & 100 & 4 & & 2 & & 3 & & 2 & & 3 & & & \\
\hline & 101 & 4 & & 2 & & 3 & & 2 & & 3 & & & \\
\hline & 102 & 4 & & 2 & & 4 & & 2 & & 2 & & & \\
\hline & 103 & 4 & & 2 & & 3 & & 2 & & 2 & & & \\
\hline & 104 & 4 & & 2 & & 4 & & 2 & & 3 & & & \\
\hline & 105 & 4 & & 2 & & 3 & & 2 & & 2 & & & \\
\hline & 106 & 4 & & 4 & & 3 & & 2 & & 3 & & & \\
\hline & 107 & 3 & & 2 & & 3 & & 2 & & 2 & & & \\
\hline & 108 & 4 & & 2 & & 3 & & 2 & & 3 & & & \\
\hline & 109 & 3 & & 4 & & 2 & & 2 & & 2 & & & \\
\hline & 110 & 4 & & 2 & & 4 & & 2 & & 3 & & & \\
\hline & 111 & 3 & & 2 & & 3 & & 2 & & 1 & & & \\
\hline \multicolumn{4}{|c|}{$\begin{array}{l}\text { Vegetation: Choose from } \\
1 \text { Unvegetated, at least } 2 / 3 \text { of shoreline is gra } \\
2 \text { Open vegetation, grass } \\
3 \text { Immature, } 2 / 3 \text { willow } \\
4 \text { Mixed willow and hardwood } \\
5 \text { Mature, at least } 2 / 3 \text { hardwood }\end{array}$} & \multicolumn{3}{|c|}{$\begin{array}{l}\text { Bank: Choose from } \\
1 \text { Cut bank with exposed soil } \\
2 \text { Cut bank, stabilized with tree roots } \\
3 \text { Sloped unvegetated bank } \\
4 \text { Sloped vegetated bank }\end{array}$} & \multicolumn{4}{|c|}{$\begin{array}{l}\text { Basking: Choose from } \\
1 \text { No basking structures } \\
2 \text { Few basking structures (less than } 5 \text { per } 50 \mathrm{~m} \text { ) } \\
3 \text { Moderate basking structures }(5 \text { to } 15 \text { per } 50 \mathrm{~m} \text { ) } \\
4 \text { High basking structures (greater than } 15 \text { per } 50 \mathrm{~m} \text { ) }\end{array}$} & \multicolumn{2}{|c|}{$\begin{array}{l}\text { Velocity: Choose from } \\
1 \text { Flat water along bank and under basking structures } \\
2 \text { Moving water along bank and under basking structures } \\
3 \text { White water along bank }\end{array}$} & $\begin{array}{l}\text { Depth: Choose from } \\
1 \text { Shallow, less than } 10 \mathrm{~cm} \text {, under basking habitat } \\
210-100 \mathrm{~cm} \text { under basking habitat } \\
3 \text { Deep, greater than } 100 \mathrm{~cm} \text {, under basking habitat }\end{array}$ \\
\hline
\end{tabular}


RIVER LEFT

Date:

\begin{tabular}{|l|l|l|l|l|}
\hline Vegetation & Bank & Basking & Velocity & Depth \\
\hline
\end{tabular}

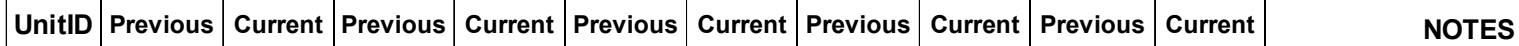

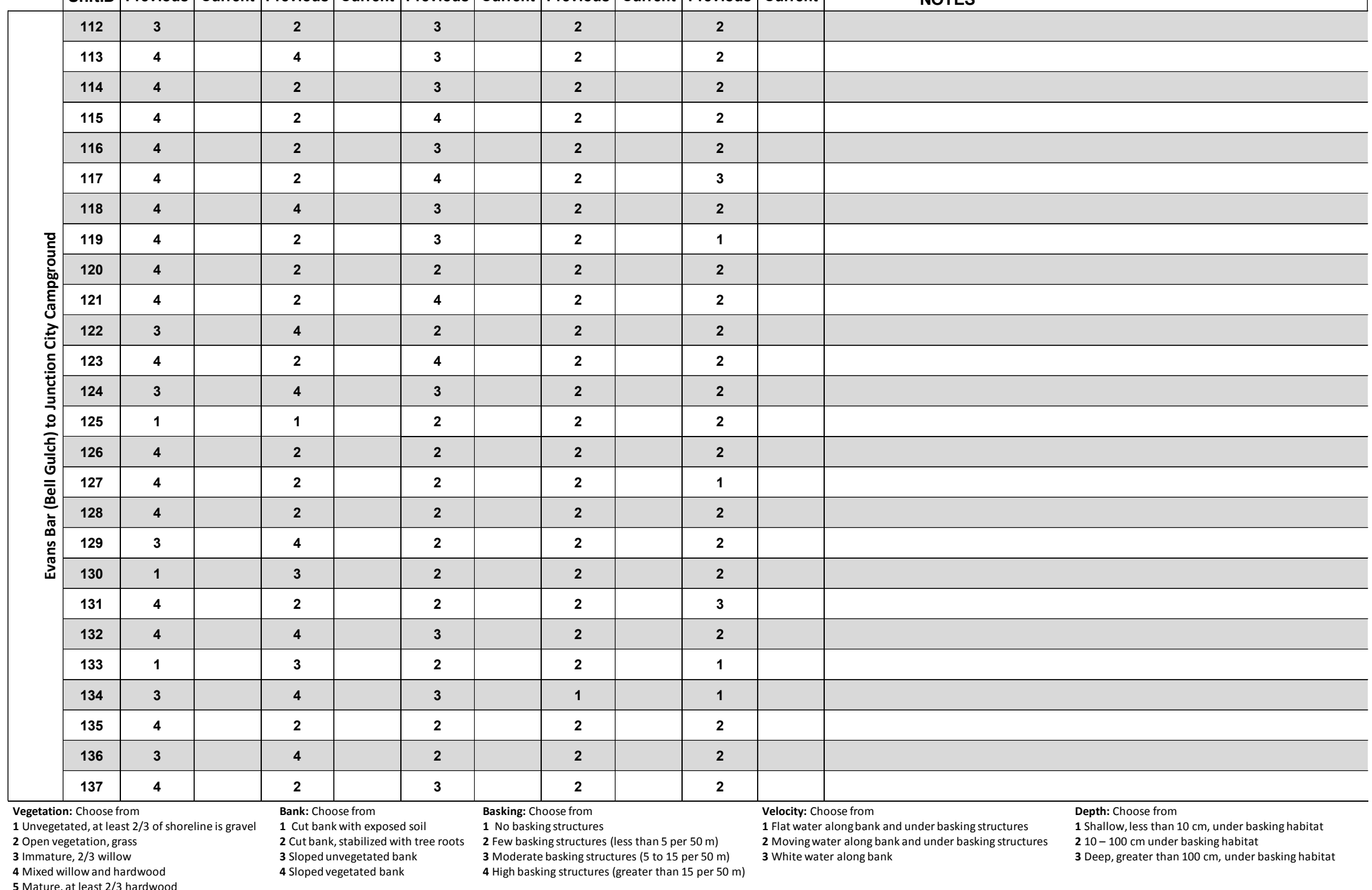




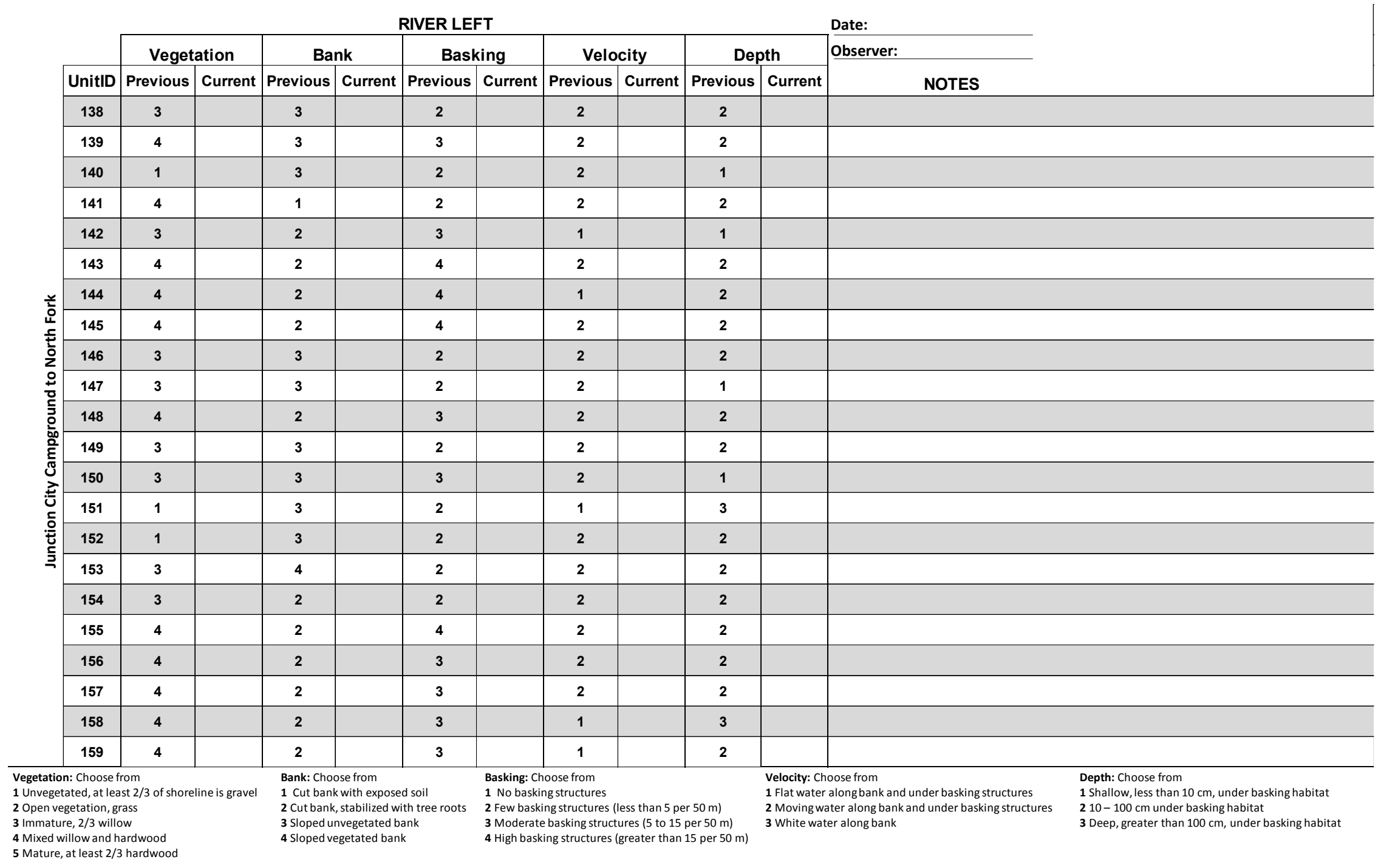


RIVER RIGHT

Date:

\begin{tabular}{|l|l|l|l|l|} 
Vegetation & Bank & Basking & Velocity & Depth \\
\hline
\end{tabular}

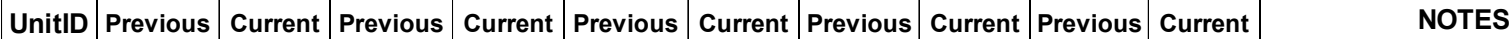

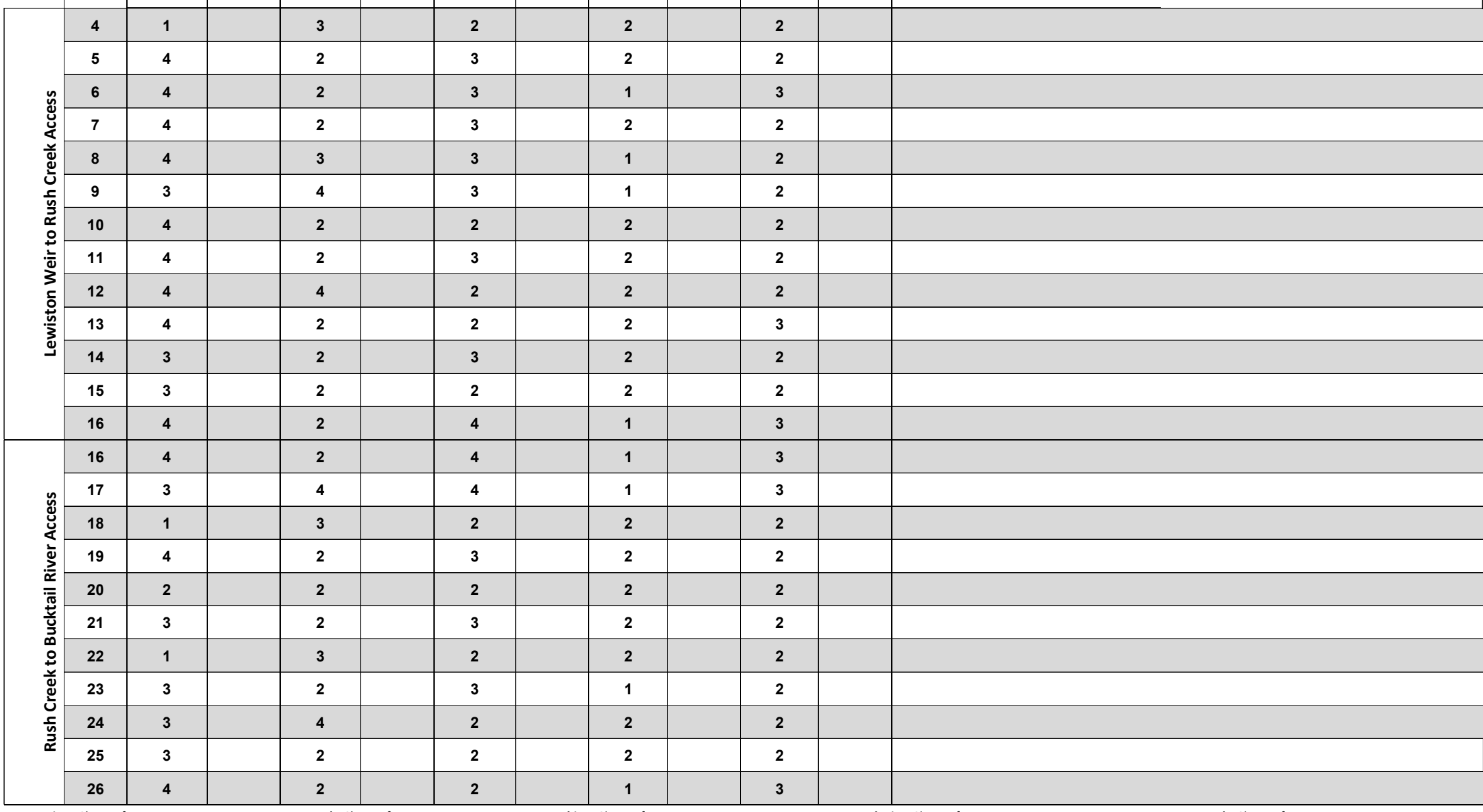

Vegetation: Choose from

1 Unvegetated, at least $2 / 3$ of shoreline is gravel

2 Open vegetation, grass

I Mixed willowand hardwood

5 Mature, at least $2 / 3$ hardwood
Bank: Choose from

1 Cut bank with exposed soil

2 Cut bank, stabilized with tree roots

4 Sloped vegetated bank
Basking: Choose from

1 No basking structures

(

4 High basking strutures (Bes 15 to 15 per $50 \mathrm{~m}$ )

1 Flat water along bank and under basking structure

2 Moving water along bank

Depth: Choose from

1 Shallow, less than $10 \mathrm{~cm}$, under basking habita

$210-100 \mathrm{~cm}$ under basking habitat

3 white water along bank 
RIVER RIGHT

Date:

\begin{tabular}{l|l|l|l|l|}
\hline Vegetation & Bank & Basking & Velocity & Depth \\
\hline
\end{tabular}

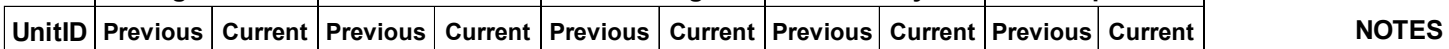

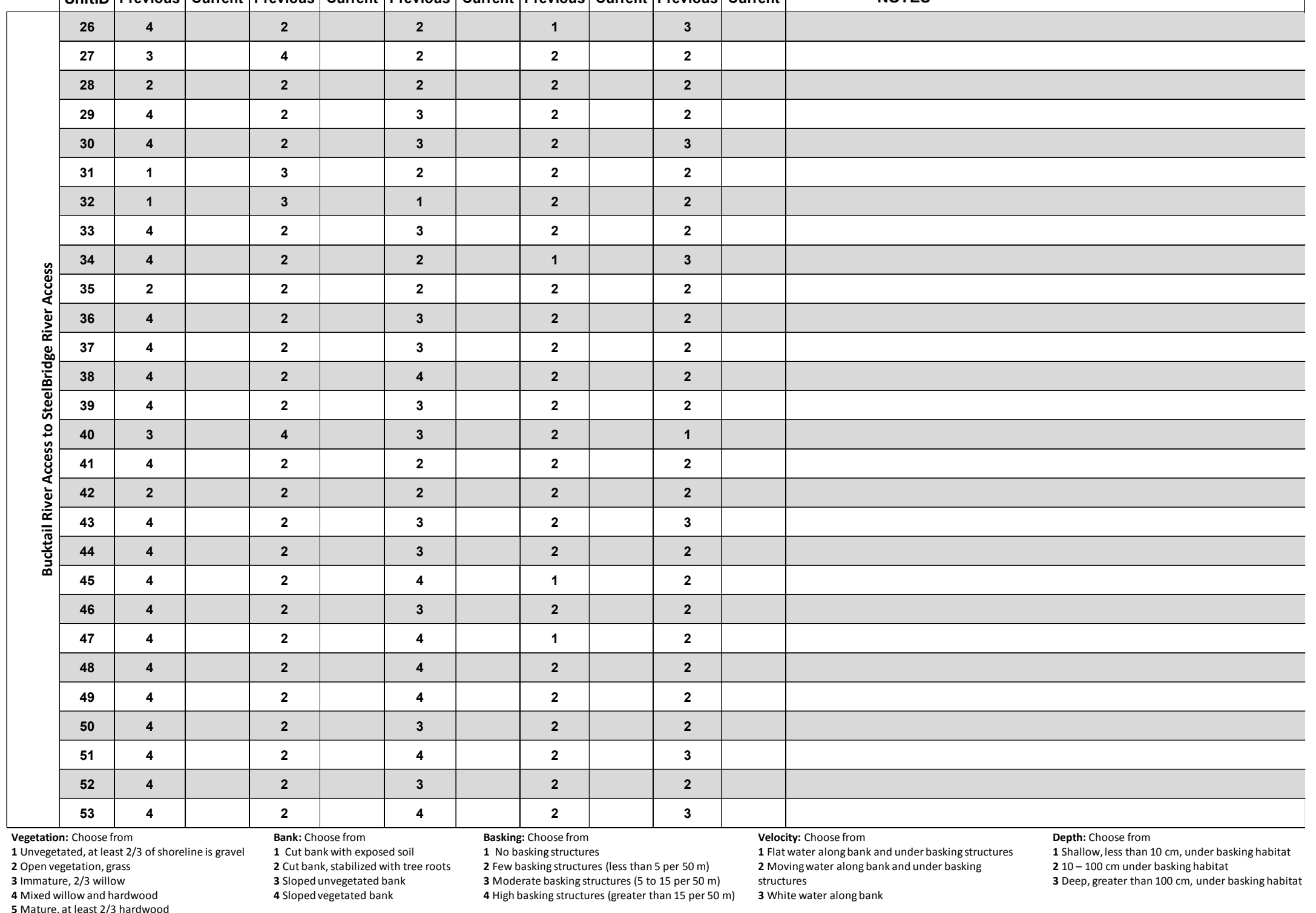




\begin{tabular}{|c|c|c|c|c|c|c|c|c|c|c|c|c|c|}
\hline & \multirow[b]{3}{*}{ UnitlD } & \multicolumn{10}{|c|}{ RIVER RIGHT } & Date: & \\
\hline & & \multicolumn{2}{|c|}{ Vegetation } & \multicolumn{2}{|c|}{ Bank } & \multicolumn{2}{|c|}{ Basking } & \multicolumn{2}{|c|}{ Velocity } & \multicolumn{2}{|c|}{ Depth } & \multirow{2}{*}{ Observer: } & \\
\hline & & Previous & Current & Previous & Current & Previous & Current & Previous & Current & Previous & Current & & \\
\hline \multirow{3}{*}{ 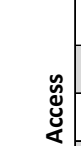 } & 53 & 4 & & 2 & & 4 & & 2 & & 3 & & & \\
\hline & 54 & 4 & & 2 & & 4 & & 2 & & 2 & & & \\
\hline & 55 & 4 & & 2 & & 4 & & 2 & & 2 & & & \\
\hline \multirow{3}{*}{ 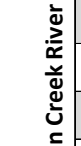 } & 56 & 4 & & 2 & & 4 & & 1 & & 3 & & & \\
\hline & 57 & 2 & & 2 & & 2 & & 1 & & 2 & & & \\
\hline & 58 & 2 & & 2 & & 2 & & 2 & & 2 & & & \\
\hline \multirow{2}{*}{ 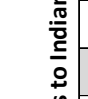 } & 59 & 4 & & 2 & & 3 & & 1 & & 2 & & & \\
\hline & 60 & 4 & & 2 & & 3 & & 1 & & 2 & & & \\
\hline \multirow{3}{*}{. } & 61 & 4 & & 4 & & 3 & & 2 & & 2 & & & \\
\hline & 62 & 4 & & 2 & & 4 & & 2 & & 2 & & & \\
\hline & 63 & 4 & & 2 & & 3 & & 2 & & 2 & & & \\
\hline \multirow{4}{*}{ 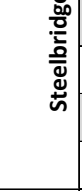 } & 64 & 4 & & 2 & & 4 & & 1 & & 2 & & & \\
\hline & 65 & 4 & & 2 & & 4 & & 2 & & 2 & & & \\
\hline & 66 & 4 & & 2 & & 4 & & 1 & & 3 & & & \\
\hline & 67 & 4 & & 2 & & 3 & & 2 & & 2 & & & \\
\hline \multirow{13}{*}{ 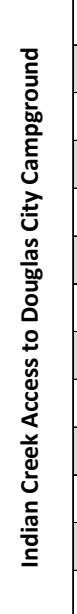 } & 67 & 4 & & 2 & & 3 & & 2 & & 2 & & & \\
\hline & 68 & 4 & & 2 & & 3 & & 2 & & 2 & & & \\
\hline & 69 & 2 & & 3 & & 3 & & 2 & & 2 & & & \\
\hline & 70 & 4 & & 2 & & 3 & & 2 & & 2 & & & \\
\hline & 71 & 3 & & 4 & & 2 & & 2 & & 2 & & & \\
\hline & 72 & 3 & & 4 & & 2 & & 2 & & 2 & & & \\
\hline & 73 & 2 & & 4 & & 2 & & 2 & & 2 & & & \\
\hline & 74 & 4 & & 4 & & 3 & & 1 & & 2 & & & \\
\hline & 75 & 4 & & 2 & & 3 & & 1 & & 2 & & & \\
\hline & 76 & 4 & & 2 & & 4 & & 2 & & 3 & & & \\
\hline & 77 & 1 & & 3 & & 2 & & 2 & & 2 & & & \\
\hline & 78 & 3 & & 4 & & 2 & & 2 & & 2 & & & \\
\hline & 79 & 2 & & 4 & & 2 & & 2 & & 2 & & & \\
\hline \multicolumn{4}{|c|}{ 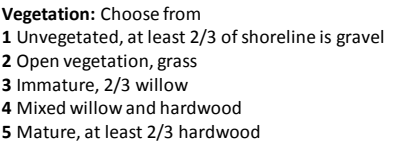 } & $\begin{array}{l}\text { Bank: Cho } \\
\text { 1 cut ban } \\
2 \text { Cut ban } \\
3 \text { Sloped } \\
4 \text { sloped }\end{array}$ & $\begin{array}{l}\text { ose from } \\
\text { kivith expos } \\
\text { k, stabilied } \\
\text { nuegetated } \\
\text { egetated bat }\end{array}$ & $\begin{array}{l}\text { ed soil } \\
\text { itth iree roots } \\
\text { ank } \\
\text { ank }\end{array}$ & & 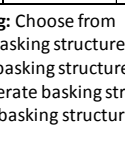 & $\begin{array}{l}\text { es } \\
\text { es } \\
\text { sultess than } \\
\text { rutures } 5 \text { to } \\
\text { res greatert }\end{array}$ & $\begin{array}{l}5 \text { per } 50 \mathrm{~m}) \\
\text { p15 per } 50 \mathrm{~m}) \\
\text { han } 15 \text { per } 50\end{array}$ & $\begin{array}{l}\text { Veloci } \\
1 \text { Flat } \\
2 \text { Mou } \\
\text { structu } \\
3 \text { Whit }\end{array}$ & $\begin{array}{l}\text { ity: Choose from } \\
\text { watera rong bank and under basking structures } \\
\text { vinwater along bank and under basking } \\
\text { ures } \\
\text { te water along bank }\end{array}$ & $\begin{array}{l}\text { Depth: Choose from } \\
1 \text { shallow, less than } 10 \mathrm{~cm} \text {, under basking habitat } \\
210-10 \mathrm{~cm} \text { under abskinh habitat } \\
3 \text { Deep, greater than } 100 \mathrm{~cm} \text {, under basking habitat }\end{array}$ \\
\hline
\end{tabular}




\begin{tabular}{|c|c|c|c|c|c|c|c|c|c|c|c|c|c|}
\hline & \multirow[b]{3}{*}{ UnitID } & \multicolumn{10}{|c|}{ RIVER RIGHT } & \multirow{2}{*}{ Date: } & \\
\hline & & \multicolumn{2}{|c|}{ Vegetation } & \multicolumn{2}{|c|}{ Bank } & \multicolumn{2}{|c|}{ Basking } & \multicolumn{2}{|c|}{ Velocity } & \multicolumn{2}{|c|}{ Depth } & & \\
\hline & & \begin{tabular}{|l|} 
Previous \\
\end{tabular} & Current & \begin{tabular}{|l|} 
Previous \\
\end{tabular} & Current & \begin{tabular}{|l|} 
Previous \\
\end{tabular} & Current & \begin{tabular}{|l|} 
Previous \\
\end{tabular} & Current & \begin{tabular}{|l|} 
Previous \\
\end{tabular} & Current & Observer: & \\
\hline \multirow{14}{*}{ 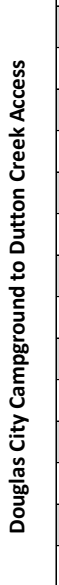 } & \begin{tabular}{|l|}
80 \\
\end{tabular} & 1 & & 3 & & 2 & & 2 & & 2 & & & \\
\hline & 81 & 4 & & 3 & & 2 & & 2 & & 2 & & & \\
\hline & 82 & 3 & & 3 & & 2 & & 2 & & 2 & & & \\
\hline & 83 & 4 & & 2 & & 3 & & 2 & & 2 & & & \\
\hline & 84 & 4 & & 2 & & 3 & & 1 & & 2 & & & \\
\hline & 85 & 4 & & 2 & & 3 & & 1 & & 2 & & & \\
\hline & 86 & 4 & & 2 & & 2 & & 1 & & 2 & & & \\
\hline & 87 & 1 & & 3 & & 2 & & 2 & & 2 & & & \\
\hline & 88 & 4 & & 2 & & 2 & & 1 & & 2 & & & \\
\hline & 89 & 1 & & 3 & & 2 & & 2 & & 2 & & & \\
\hline & 90 & 4 & & 2 & & 3 & & 2 & & 2 & & & \\
\hline & 91 & 4 & & 2 & & 3 & & 2 & & 2 & & & \\
\hline & 92 & 4 & & 2 & & 3 & & 1 & & 3 & & & \\
\hline & 93 & 4 & & 2 & & 3 & & 2 & & 3 & & & \\
\hline \multirow{19}{*}{ 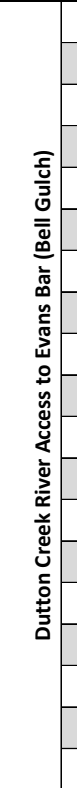 } & 93 & 4 & & 2 & & 3 & & 2 & & 3 & & & \\
\hline & 94 & 4 & & 2 & & 3 & & 2 & & 3 & & & \\
\hline & 95 & 4 & & 2 & & 3 & & 2 & & 2 & & & \\
\hline & 96 & 4 & & 2 & & 3 & & 1 & & 3 & & & \\
\hline & 97 & 3 & & 2 & & 3 & & 1 & & 3 & & & \\
\hline & 98 & 3 & & 4 & & 2 & & 2 & & 3 & & & \\
\hline & 99 & 4 & & 2 & & 3 & & 2 & & 3 & & & \\
\hline & 100 & 3 & & 2 & & 3 & & 1 & & 3 & & & \\
\hline & 101 & 1 & & 3 & & 2 & & 2 & & 3 & & & \\
\hline & 102 & 4 & & 2 & & 3 & & 2 & & 3 & & & \\
\hline & 103 & 4 & & 2 & & 4 & & 2 & & 2 & & & \\
\hline & 104 & 3 & & 4 & & 3 & & 2 & & 3 & & & \\
\hline & 105 & 3 & & 4 & & 3 & & 1 & & 2 & & & \\
\hline & 106 & 3 & & 4 & & 3 & & 1 & & 3 & & & \\
\hline & 107 & 4 & & 2 & & 4 & & 2 & & 2 & & & \\
\hline & 108 & 4 & & 2 & & 2 & & 2 & & 2 & & & \\
\hline & 109 & 1 & & 3 & & 1 & & 2 & & 2 & & & \\
\hline & 110 & 4 & & 2 & & 3 & & 2 & & 2 & & & \\
\hline & 111 & 4 & & 4 & & 3 & & 2 & & 2 & & & \\
\hline & 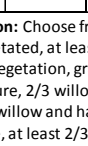 & $\begin{array}{l}\text { trom } \\
\text { tros } 2 / 3 \text { of shor } \\
\text { ars } \\
\text { orardwood } \\
\text { shardwood }\end{array}$ & is gravel & \multicolumn{3}{|c|}{ 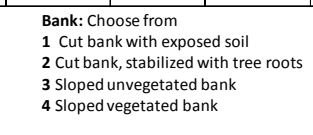 } & $\begin{array}{l}\text { Baskin } \\
1 \text { Nob } \\
2 \text { Few } \\
3 \text { Mod } \\
4 \text { High }\end{array}$ & 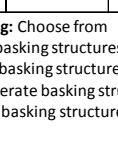 & es & $\begin{array}{l}5 \text { per } 50 \mathrm{~m} \\
15 \text { er } 50\end{array}$ & \multicolumn{2}{|c|}{ 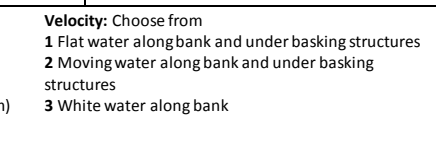 } & $\begin{array}{l}\text { Depth: Choose from } \\
1 \text { Shallow, less than } 10 \mathrm{~cm} \text {, under basking habitat } \\
210-10 \mathrm{com} \text { under baskinh habititat } \\
3 \text { Deep, greater than } 100 \mathrm{~cm} \text {, under basking habitat }\end{array}$ \\
\hline
\end{tabular}


RIVER RIGHT

Date:

\begin{tabular}{|l|l|l|l|l|}
\hline Vegetation & Bank & Basking & Velocity & Depth \\
\hline
\end{tabular}

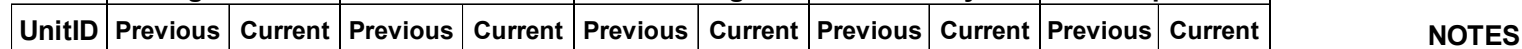

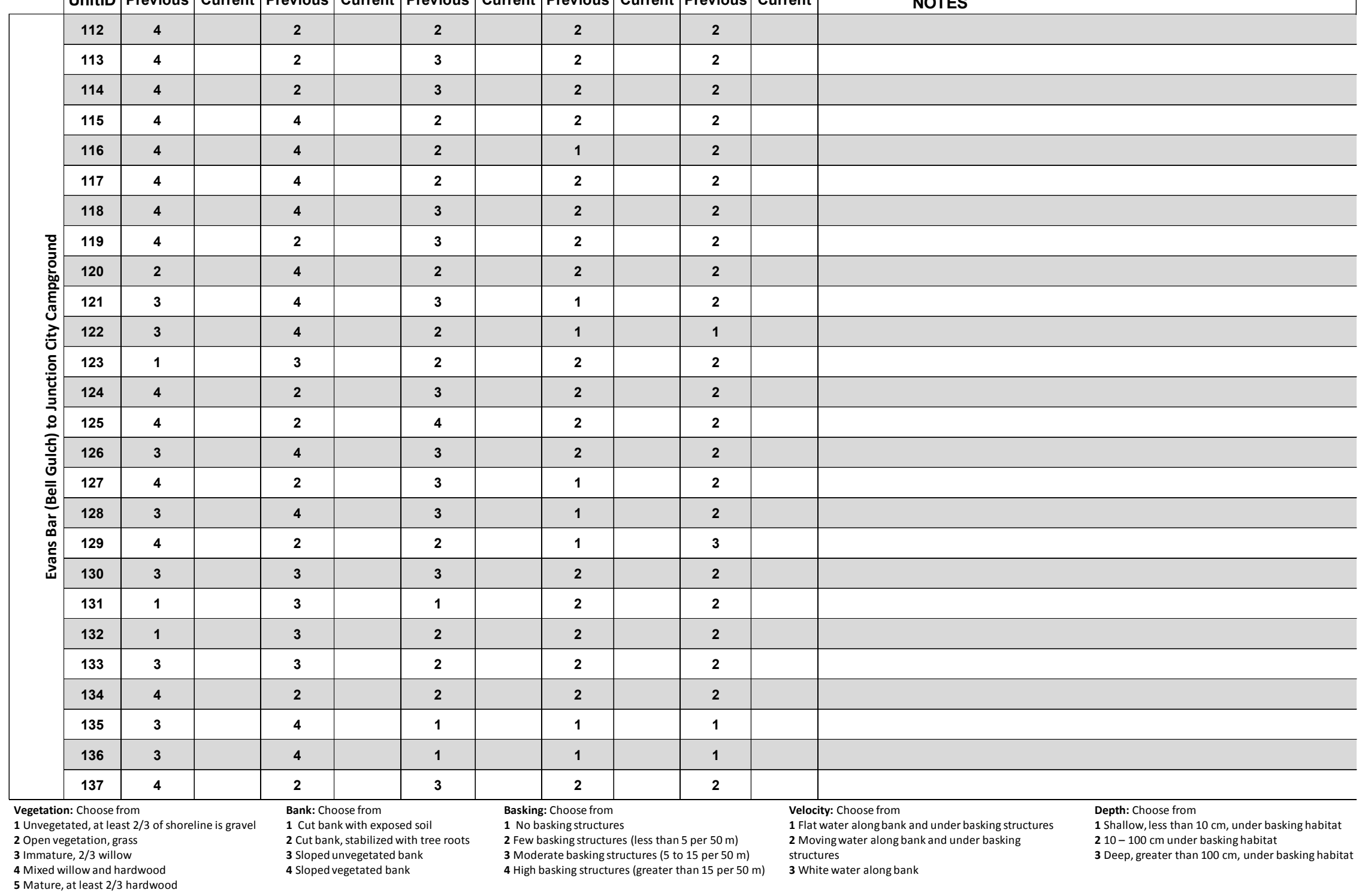




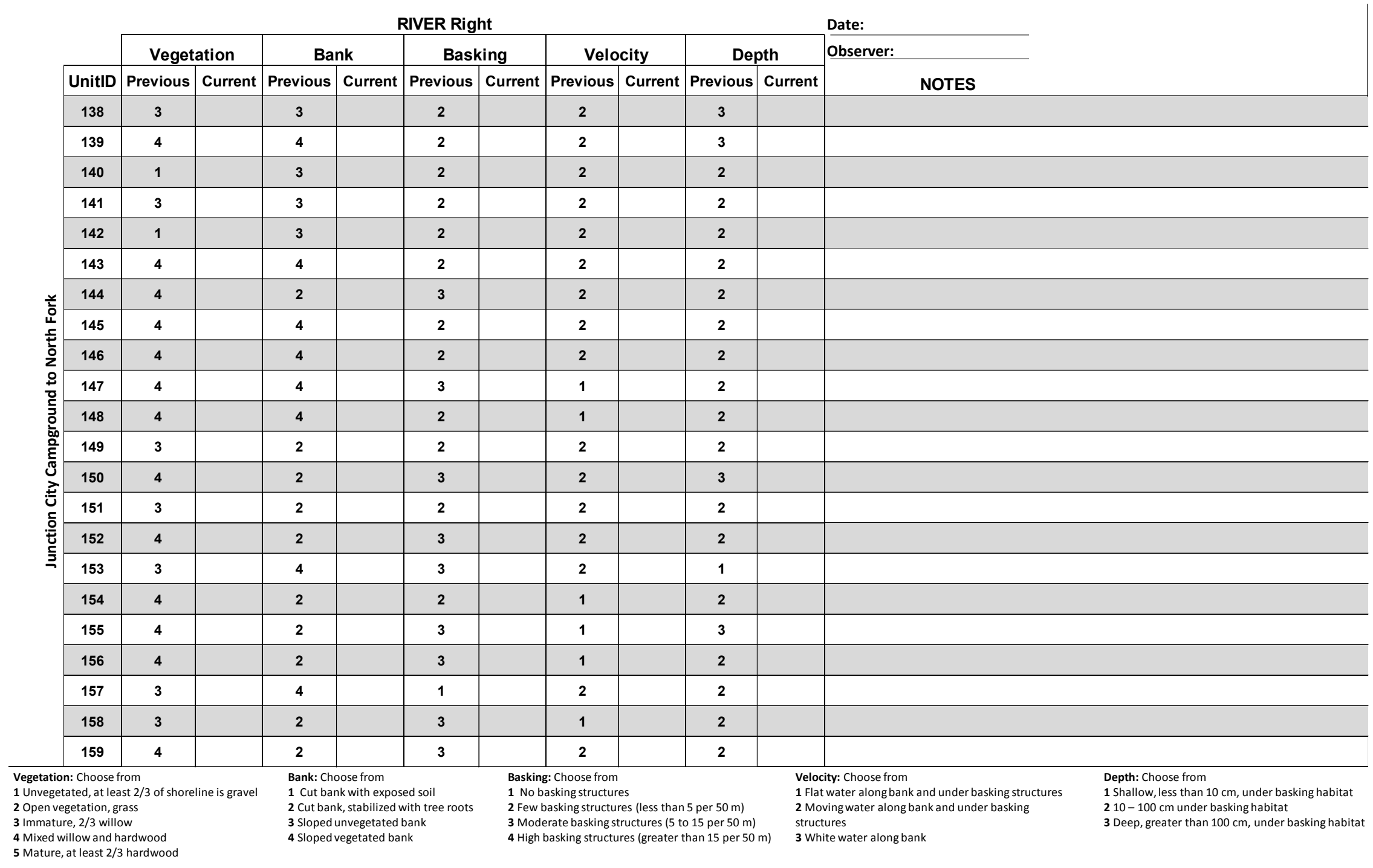


Datasheet 2: use for subsequent Main Stem surveys once habitat is characterized for each unit

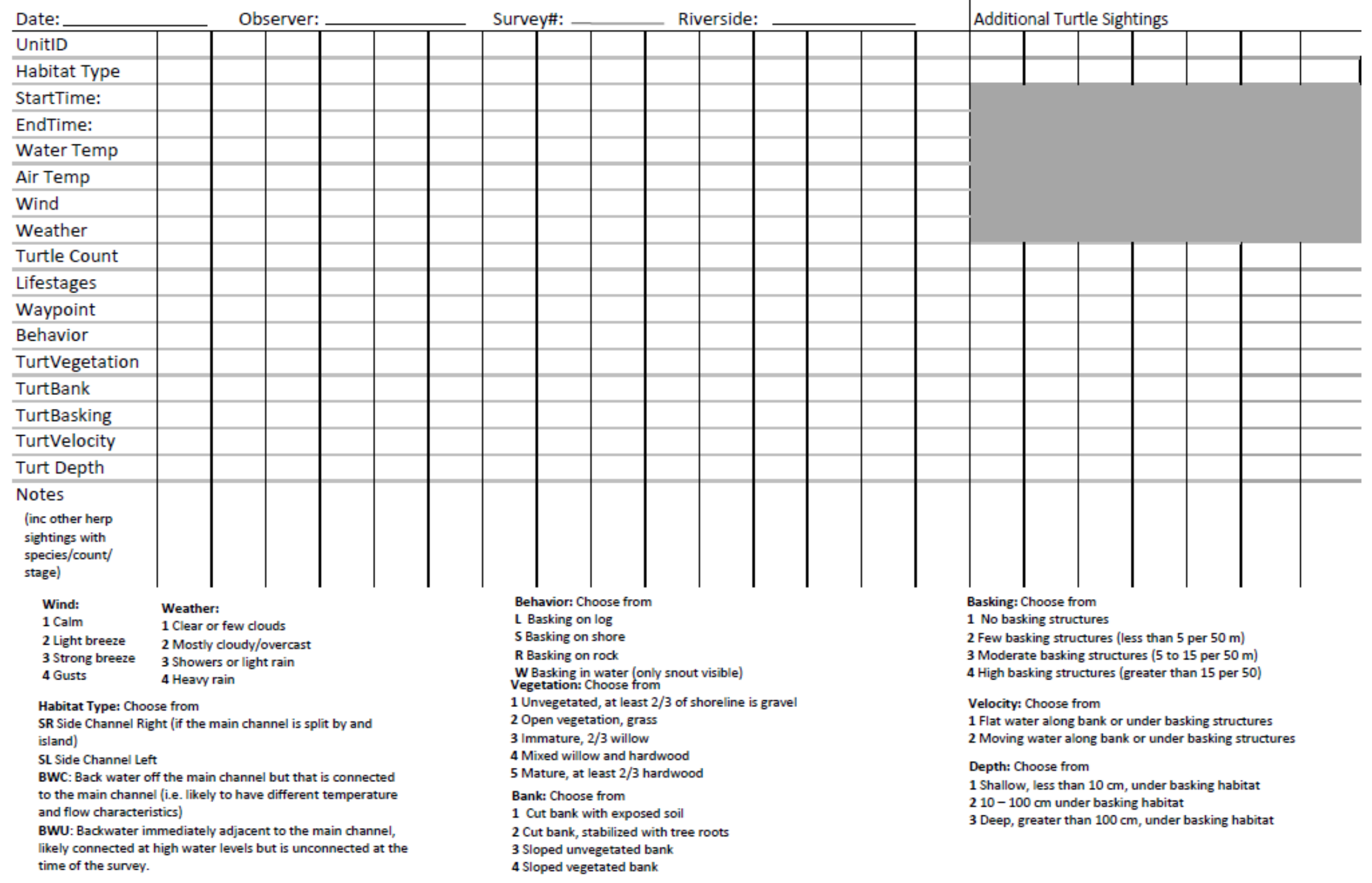


Datasheet 3: habitat characteristics for pond surveys

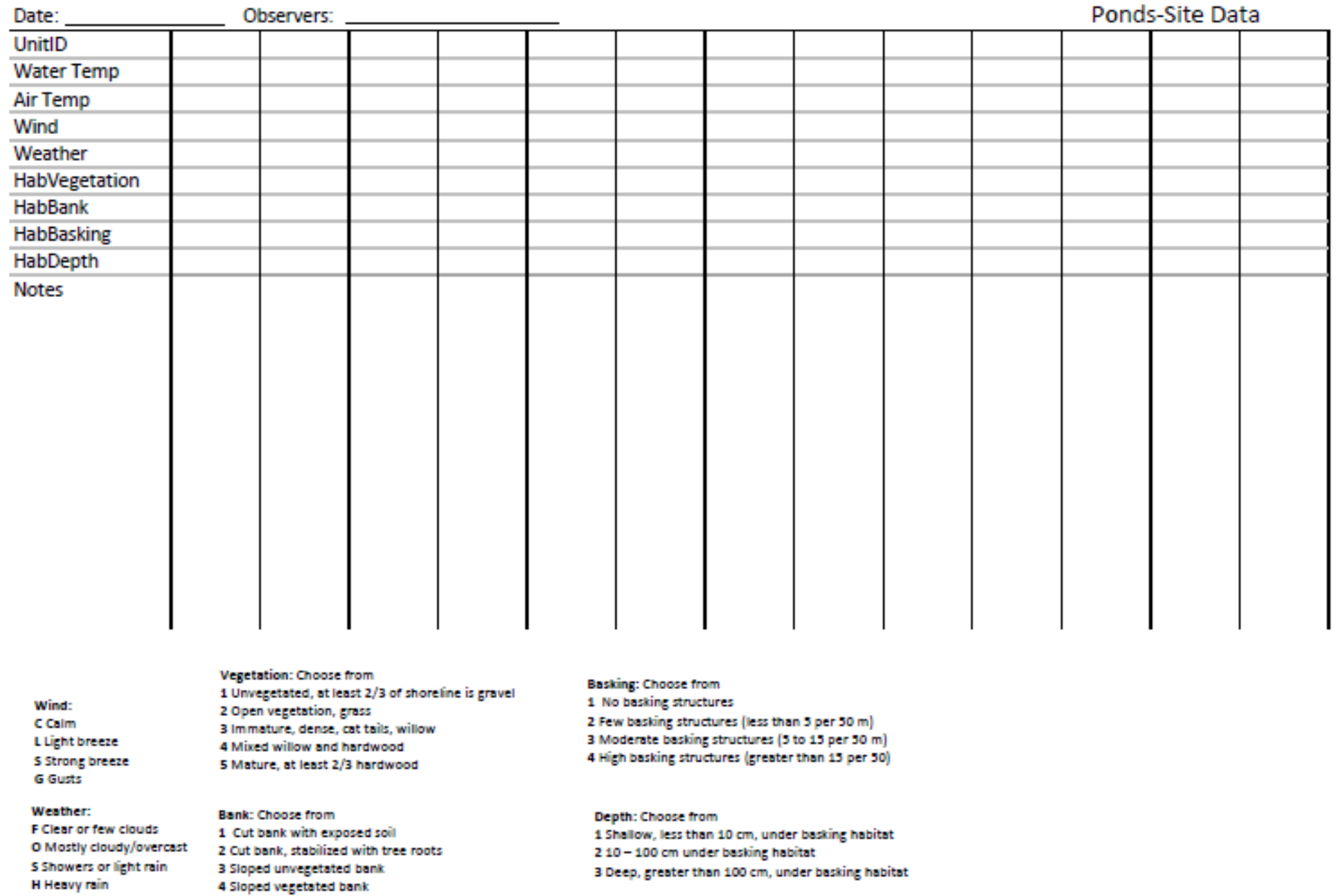




\section{Datasheet 4: Turtle observations at ponds}

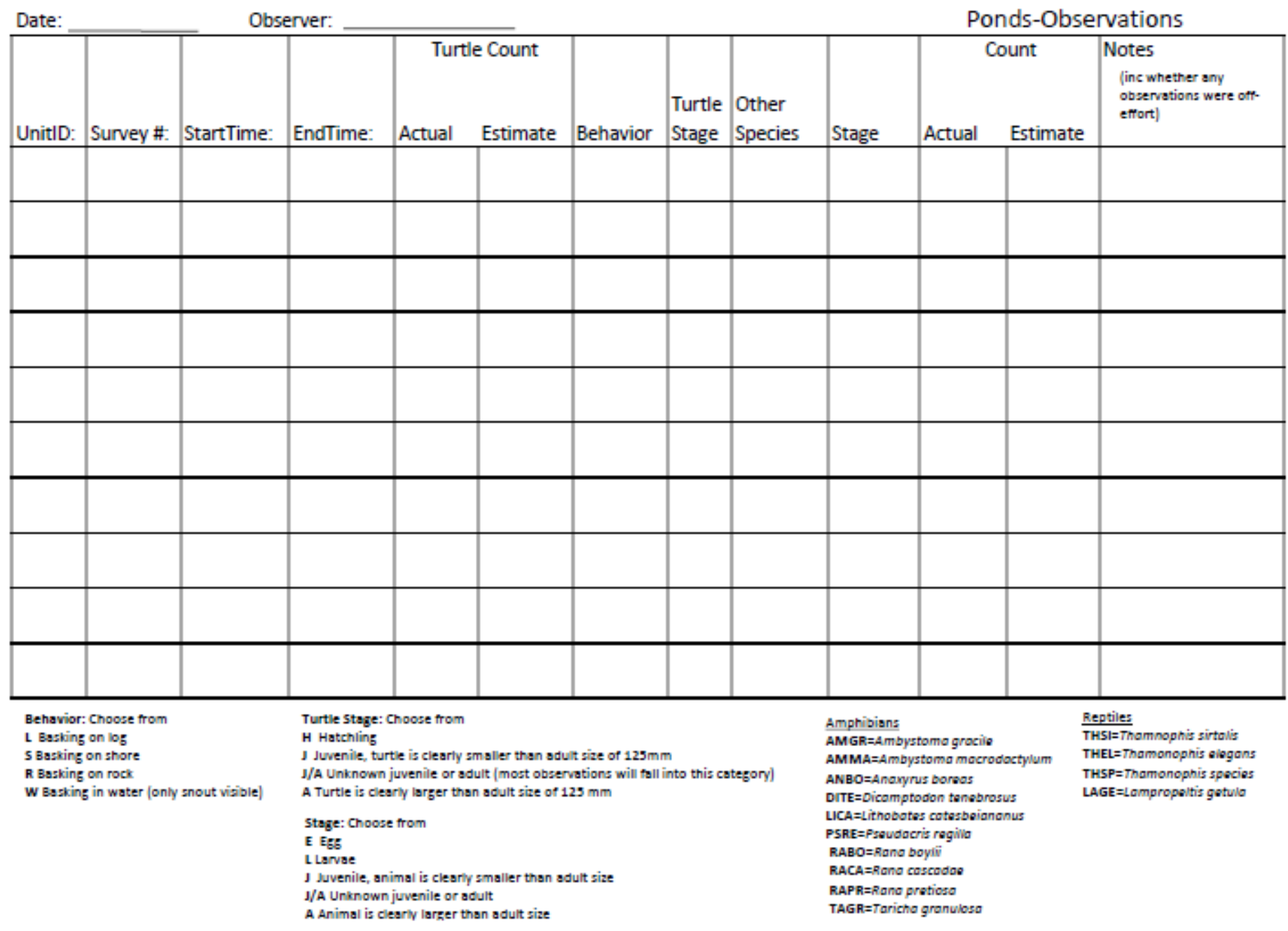




\section{Appendix H. Pond Location and Information for Western Pond Turtle Surveys}

\begin{tabular}{|c|c|c|c|}
\hline ID & UTMN & UTME & Notes \\
\hline RW-5a & 4504707 & 512005 & Lowden Ranch, get key from TRRP \\
\hline$R W-5 b$ & 4504687 & 512094 & Lowden Ranch, get key from TRRP \\
\hline RW-3 & 4504767 & 512209 & Lowden Ranch, get key from TRRP \\
\hline FEW-3 & 4504954 & 512352 & Lowden Ranch, get key from TRRP \\
\hline PD-2 & 4505134 & 512484 & Lowden Ranch, get key from TRRP \\
\hline Hamilton & 4504444 & 511939 & Lowden Ranch, get key from TRRP \\
\hline Pvt 1a & 4505357 & 512859 & Diane Gannon landowner, sheep farm, 2872 Lewiston Rd, don't have a phone number \\
\hline Pvt $1 b$ & 4505375 & 512897 & Diane Gannon landowner, sheep farm, 2872 Lewiston Rd, don't have a phone number, \\
\hline Bucktail & 4506238 & 513228 & $\begin{array}{l}\text { At the Bucktail River Access } \\
\text { Chuck and Liz Johnson, Donmovin Rd (private) off of Sky Ranch, call first 530-623-4147 or 530- }\end{array}$ \\
\hline Pvt 4a & 4507296 & 496589 & $\begin{array}{l}\text { 739-3639 } \\
\text { Chuck and Liz Johnson, Donmovin Rd (private) off of Sky Ranch, call first 530-623-4147 or 530- }\end{array}$ \\
\hline Pvt $4 b$ & 4507337 & 496536 & $739-3639$ \\
\hline Pvt 5d & 4506277 & 496127 & No landowner issue (absent owner), long hike in, maybe get at it from the river \\
\hline JC BLM & 4508068 & 496030 & At the Sky Ranch River Access, near the road \\
\hline NHD1 & 4511673 & 494597.7 & Across highway from McCarthy, up Power House Road a short way \\
\hline NHD2 & 4511674 & 494260.1 & McCarthy Pond, no landowner issue \\
\hline NHD3 & 4511563 & 494175.6 & Between McCarthy and the river, no landowner issue \\
\hline NHD12 & 4506134 & 513938.2 & Salt Flats near Bucktail \\
\hline NHD13 & 4506134 & 514022.7 & Salt Flats near Bucktail \\
\hline Lorenz & 4502087.1 & 502806.3 & River left at restoration site, near channel \\
\hline JC Rehab & 4508336.1 & 495348.6 & River left at upstream end of restoration site \\
\hline
\end{tabular}




\section{Appendix I. Gosner Stage Tables for Frog Embryo and Larva}
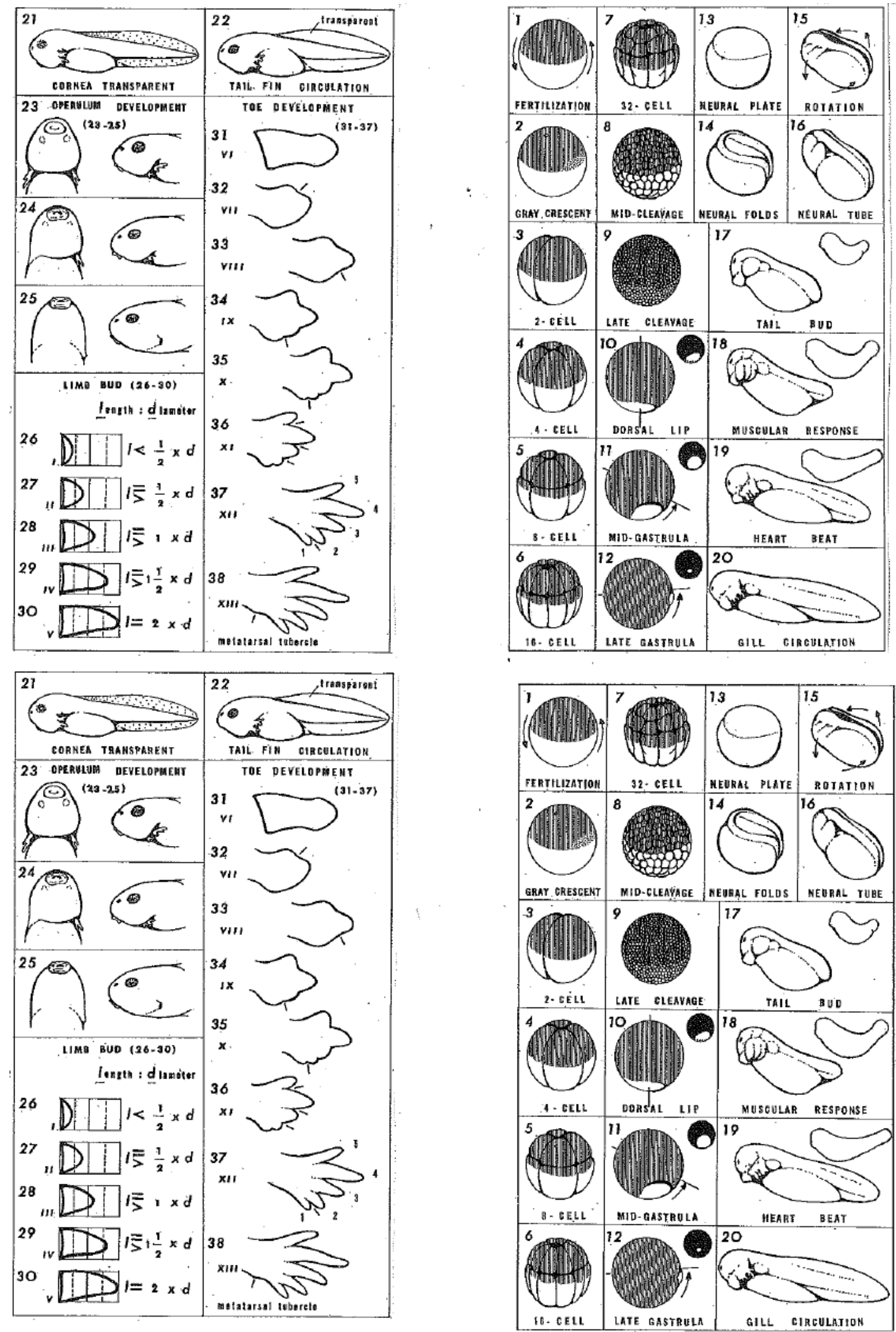


\section{Appendix J. Input Data Structure for R Script: Foothill Yellow-Legged Frogs}

[Table shows the column headers and data for the first three sites, and the definition of each column. Data have been transposed for better display as a table, in the comma separate values (.csv) file, the headers appear on the first row, and data for each site in the subsequent rows. The 'Definitions' are not part of the .csv file and are only given here for clarification]

\begin{tabular}{|c|c|c|c|c|}
\hline Header & Data Site1 & Data Site 2 & Data Site 3 & Definition \\
\hline $\mathrm{y} .1$ & 0 & 0 & 0 & Detections Survey 1, Season 1 \\
\hline $\mathrm{y} .2$ & 0 & 0 & 0 & Detections Survey 2, Season 1 \\
\hline$y .3$ & 0 & 0 & 0 & Detections Survey 1, Season 2 \\
\hline $\mathrm{y} .4$ & 0 & 0 & 0 & Detections Survey 2, Season 2 \\
\hline $\mathrm{y} .5$ & 0 & 0 & 0 & Detections Survey 1, Season 3 \\
\hline y. 6 & 0 & 0 & 0 & Detections Survey 2 Season 3 \\
\hline Date1 & 41442 & 41442 & 41442 & Date Survey 1, Season 1 \\
\hline Date2 & 41467 & 41467 & 41467 & Date Survey 2, Season 1 \\
\hline Date3 & 41813 & 41813 & 41813 & Date Survey 1, Season 2 \\
\hline Date4 & 41821 & 41821 & 41821 & Date Survey 2, Season 2 \\
\hline Date5 & 42174 & 42174 & 42174 & Date Survey 1, Season 3 \\
\hline Date6 & 42191 & 42191 & 42191 & Date Survey 2, Season 3 \\
\hline Water 1 & 13.0 & 12.5 & 13.0 & Water temperature Survey 1 , Season 1 \\
\hline Water2 & 10.6 & 10.8 & 11.0 & Water temperature Survey 2, Season 1 \\
\hline Water3 & 11.7 & 12.0 & 12.3 & Water temperature Survey 1, Season 2 \\
\hline Water4 & 12.2 & 12.5 & 12.8 & Water temperature Survey 2, Season 2 \\
\hline Water5 & 11.5 & 12.0 & 12.5 & Water temperature Survey 1, Season 3 \\
\hline Water6 & 15.8 & 16.3 & 17.5 & Water temperature Survey 2 Season 3 \\
\hline Air1 & 25.0 & 25.0 & 26.0 & Air temperature Survey 1, Season 1 \\
\hline Air2 & 16.0 & 20.0 & 21.0 & Air temperature Survey 2, Season 1 \\
\hline Air3 & 19.9 & 22.5 & 23.6 & Air temperature Survey 1, Season 2 \\
\hline Air4 & 21.0 & 26.2 & 24.3 & Air temperature Survey 2, Season 2 \\
\hline Air5 & 16.7 & 21.8 & 22.8 & Air temperature Survey 1, Season 3 \\
\hline Air6 & 30.8 & 32.3 & 31.0 & Air temperature Survey 2 Season 3 \\
\hline Wind1 & 3 & 2 & 2 & Wind Survey 1, Season 1 \\
\hline Wind2 & 1 & 1 & 1 & Wind Survey 2, Season 1 \\
\hline Wind3 & 1 & 1 & 1 & Wind Survey 1, Season 2 \\
\hline Wind4 & 1 & 1 & 1 & Wind Survey 2, Season 2 \\
\hline Wind5 & 1 & 2 & 2 & Wind Survey 1, Season 3 \\
\hline Wind6 & 2 & 2 & 2 & Wind Survey 2 Season 3 \\
\hline Weather1 & 1 & 1 & 1 & Weather Survey 1, Season 1 \\
\hline Weather2 & 1 & 1 & 1 & Weather Survey 2, Season 1 \\
\hline Weather3 & 1 & 1 & 1 & Weather Survey 1, Season 2 \\
\hline Weather4 & 1 & 1 & 1 & Weather Survey 2, Season 2 \\
\hline Weather5 & 1 & 1 & 1 & Weather Survey 1, Season 3 \\
\hline
\end{tabular}




\begin{tabular}{|c|c|c|c|c|}
\hline Header & Data Site1 & Data Site 2 & Data Site 3 & Definition \\
\hline Weather6 & 2 & 1 & 1 & Weather Survey 2 Season 3 \\
\hline Gravel1 & 58 & 46 & 0 & Amount of breeding habitat $(\mathrm{m})$ Season 1 \\
\hline Gravel2 & 81.11468 & 77.434 & 174.2718 & Amount of breeding habitat $(\mathrm{m})$ Season 2 \\
\hline Gravel3 & 233.8 & 114.32 & 466.91 & $\begin{array}{l}\text { Amount of breeding habitat }(\mathrm{m}) \text { Season } 3 \\
\text { Percent of habitat in main channel }(\mathrm{m}) \text { Season }\end{array}$ \\
\hline PctMc1 & 1 & 1 & 0 & $\begin{array}{l}1 \\
\text { Percent of habitat in main channel (m) Season }\end{array}$ \\
\hline PctMc2 & 1 & 1 & 0.887448 & $\begin{array}{l}2 \\
\text { Percent of habitat in main channel (m) Season }\end{array}$ \\
\hline PctMc3 & 1 & 1 & 0.878777 & 3 \\
\hline DistUp & 99999 & 99999 & 99999 & Distance to nearest upstream inhabited trib (m) \\
\hline
\end{tabular}




\section{Appendix K. Input Data Structure for R script, Western Pond Turtles}

[Table shows the column headers and data for the first three sites, and the definition of each column. Data have been transposed for better display as a table, in the comma separate values (.csv) file, the headers appear on the first row, and data for each site in the subsequent rows. The 'Definitions' are not part of the .csv file and are only given here for clarification]

\begin{tabular}{|c|c|c|c|c|}
\hline Header & Data Site1 & Data Site 2 & Data Site 3 & Definition \\
\hline y.1 & 0 & 0 & 0 & Detections Survey 1, Season 1 \\
\hline y. 2 & 0 & 0 & 0 & Detections Survey 2, Season 1 \\
\hline y.3 & 0 & 0 & 0 & Detections Survey 3, Season 1 \\
\hline y.4 & 0 & 0 & 0 & Detections Survey 1, Season 2 \\
\hline y.5 & 0 & 0 & 0 & Detections Survey 2, Season 2 \\
\hline y.6 & 0 & 0 & 0 & Detections Survey 3, Season 2 \\
\hline y.7 & 0 & 0 & 0 & Detections Survey 1, Season 3 \\
\hline y.8 & 0 & 0 & 0 & Detections Survey 2 Season 3 \\
\hline y.9 & 0 & 0 & 0 & Detections Survey 3 Season 3 \\
\hline Date1 & 41480 & 41480 & 41480 & Date Survey 1, Season 1 \\
\hline Date2 & 41485 & 41485 & 41485 & Date Survey 2, Season 1 \\
\hline Date3 & 41489 & 41489 & 41489 & Date Survey 3, Season 1 \\
\hline Date4 & 41830 & 41830 & 41830 & Date Survey 1, Season 2 \\
\hline Date5 & 41836 & 41836 & 41836 & Date Survey 2, Season 2 \\
\hline Date6 & 41843 & 41843 & 41843 & Date Survey 3, Season 2 \\
\hline Date7 & 42194 & 42194 & 42194 & Date Survey 1, Season 3 \\
\hline Date8 & 42201 & 42201 & 42201 & Date Survey 2, Season 3 \\
\hline Date9 & 42204 & 42204 & 42204 & Date Survey 3, Season 3 \\
\hline Water1 & 9 & 9 & 9 & Water temperature Survey 2 Season 3 \\
\hline Water2 & 10 & 10 & 11 & Water temperature Survey 2 Season 3 \\
\hline Water3 & 14 & 10 & 10 & Water temperature Survey 2 Season 3 \\
\hline Water4 & 10.5 & 10.5 & 10.5 & Water temperature Survey 2 Season 3 \\
\hline Water5 & 10.7 & 10.7 & 10.7 & Water temperature Survey 2 Season 3 \\
\hline Water6 & 10.8 & 10.9 & 11 & Water temperature Survey 2 Season 3 \\
\hline Water7 & 12 & 12 & 12 & Water temperature Survey 2 Season 3 \\
\hline Water8 & 12 & 12 & 12 & Water temperature Survey 2 Season 3 \\
\hline Water9 & 12.1 & 12 & 12.1 & Water temperature Survey 2 Season 3 \\
\hline Air1 & 17 & 17 & 18 & Air temperature Survey 2 Season 3 \\
\hline Air2 & 25 & 25 & 25 & Air temperature Survey 2 Season 3 \\
\hline Air3 & 30 & 22 & 22 & Air temperature Survey 2 Season 3 \\
\hline Air4 & 17.7 & 18.7 & 20.9 & Air temperature Survey 2 Season 3 \\
\hline Air5 & 17.8 & 18.1 & 18.7 & Air temperature Survey 2 Season 3 \\
\hline Air6 & 18.7 & 18.7 & 19.8 & Air temperature Survey 2 Season 3 \\
\hline Air7 & 18.6 & 17.7 & 16.6 & Air temperature Survey 2 Season 3 \\
\hline Air8 & 18.7 & 14.7 & 15.8 & Air temperature Survey 2 Season 3 \\
\hline Air9 & 0 & 18.1 & 19.1 & Air temperature Survey 2 Season 3 \\
\hline
\end{tabular}




\begin{tabular}{lllll}
\hline \multicolumn{1}{c}{ Header } & Data Site1 & Data Site 2 & Data Site 3 & \multicolumn{1}{c}{ Definition } \\
\hline Wind1 & 1 & 1 & 1 & Wind Survey 1, Season 1 \\
Wind2 & 1 & 1 & 1 & Wind Survey 2, Season 1 \\
Wind3 & 1 & 1 & 1 & Wind Survey 3, Season 1 \\
Wind4 & 1 & 1 & 1 & Wind Survey 1, Season 2 \\
Wind5 & 1 & 1 & 1 & Wind Survey 2, Season 2 \\
Wind6 & 2 & 2 & 2 & Wind Survey 3, Season 2 \\
Wind7 & 1 & 1 & 1 & Wind Survey 1, Season 3 \\
Wind8 & 1 & 1 & 1 & Wind Survey 2, Season 3 \\
Wind9 & 1 & 1 & 2 & Wind Survey 3, Season 3 \\
Weather1 & 1 & 1 & 1 & Weather Survey 1, Season 1 \\
Weather2 & 2 & 2 & 2 & Weather Survey 2, Season 1 \\
Weather3 & 1 & 1 & 1 & Weather Survey 3, Season 1 \\
Weather4 & 1 & 1 & 1 & Weather Survey 1, Season 2 \\
Weather5 & 2 & 2 & 1 & Weather Survey 2, Season 2 \\
Weather6 & 2 & 2 & 1 & Weather Survey 3, Season 2 \\
Weather7 & 2 & 2 & 2 & Weather Survey 1, Season 3 \\
Weather8 & 1 & 1 & 1 & Weather Survey 2, Season 3 \\
Weather9 & 1 & 1 & 1 & Weather Survey 3, Season 3 \\
VegetationL & 3 & 3 & 2 & Left bank vegetation \\
BankL & 2 & 2 & 1 & Left bank composition \\
BaskingL & 2 & 2 & 1 & Left bank density of basking structures \\
VelocityL & 2 & 2 & 2 & Left bank water velocity \\
DepthL & 3 & 3 & 3 & Left bank water depth \\
VegetationR & 1 & 3 & 3 & Right bank vegetation \\
BankR & 1 & 3 & 2 & Right bank density of basking structures \\
BaskingR & 1 & 2 & 2 & Right bank water velocity \\
VelocityR & 2 & 2 & Right bank water depth \\
DepthR & 3 & 3 & Right bank density of basking structures \\
Dist & 4 & 5 & Distance to Lewiston Dam (100X m) \\
\hline & & 3 &
\end{tabular}




\section{Appendix L. R Script for Foothill Yellow-Legged Frog Occupancy Model \# \\ \# Script for conducting a multi-year occupancy model using the package unmarked \\ \# for Trinity River Foothill yellow-legged frog breeding \\ \# This program was used to produce the estimates in the report: \\ \# Snover, M.L., and Adams, M.J., 2016, Herpetological monitoring and assessment on the Trinity River, Trinity \\ Country, California—Final report: U.S. Geological Survey Open-File Report 2016-1089, 93 p. \\ \# See report for a description of the variables.

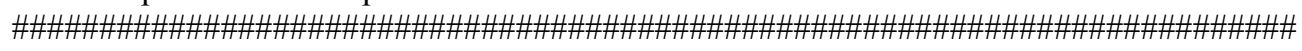

\#\#Load the libraries needed for this analysis

library(unmarked)

\#\#Set the working directory for the location of the input files, this will need to be changed to run setwd( "C:/Users/Melissa/USGS_C_Drive/R_Work/RABOAbundance")

\#\#Read in the data, observations and covariates

SurveyData<-read.csv(file="RABO_Occu_2013_2015GRTS.csv",head=TRUE,sep=",")

\#\# Tell $\mathrm{R}$ what the observations are (1/0 for detected or not detected at each survey)

breed.y<-SurveyData[,c("y.1","y.2","y.3","y.4","y.5","y.6")]

\#Standarize the continuous observation (survey-specific) covariates

DATE $<$ - as.matrix(SurveyData[,7:12])

sd.DATE $<-$ sd(c(DATE), na.rm=TRUE)

mean.DATE $<-$ mean(DATE, na.rm=TRUE)

DATE $<-($ DATE - mean.DATE $) /$ sd.DATE

Water <- as.matrix(SurveyData[,13:18])

sd.Water $<-\operatorname{sd}(\mathrm{c}($ Water $)$, na.rm=TRUE)

mean. Water $<-$ mean (Water, na.rm $=$ TRUE)

Water $<$ - (Water - mean.Water) / sd.Water

Air $<$ - as.matrix(SurveyData[,19:24])

sd.Air $<-\operatorname{sd}(\mathrm{c}$ (Air), na.rm=TRUE)

mean.Air $<$ - mean(Air, na.rm=TRUE)

Air $<-$ (Air - mean. Air) $/$ sd.Air

\#Standardize the Site-specific Covariates

DistUp $<$-as.matrix(SurveyData[,49])

sd.DistUp <- sd(c(DistUp), na.rm=TRUE)

mean.DistUp <- mean(DistUp, na.rm=TRUE)

DistUp <- (DistUp - mean.DistUp) / sd.DistUp

\#Standardize the Yearly site covariates

Gravel<-as.matrix(SurveyData[,37:39])

sd.Gravel <- sd(c(Gravel), na.rm=TRUE)

mean.Gravel <- mean(Gravel, na.rm=TRUE)

Gravel <- (Gravel - mean.Gravel) / sd.Gravel

PctMc $<$-as.matrix(SurveyData[,40:42])

sd.PctMc $<-$ sd(c(PctMc), na.rm=TRUE)

mean.PctMc $<-$ mean (PctMc, na.rm=TRUE)

PctMc <- $($ PctMc - mean.PctMc) / sd.PctMc

\#Define the years 
years $<-$ as.character(2013:2015)

years $<-$ matrix (years, nrow(SurveyData), 3, byrow=TRUE)

\#Create the unmarked dataframe for a multi-year occupancy analysis

spotUMF $<$-unmarkedMultFrame( $\mathrm{y}=$ breed.y,

siteCovs=data.frame(distUP=DistUp, gravel1=SurveyData[,c("Gravel1")]),

yearlySiteCovs=list (year=years, gravel $=$ Gravel,pctmc $=$ PctMc),

obsCovs $=$ list $($ date $=$ DATE, water $=$ Water,

air=Air,Wind=SurveyData[,c("Wind1","Wind2","Wind3","Wind4","Wind5","Wind6")],Weather=SurveyData[,c("

Weather1","Weather2","Weather3","Weather4","Weather5","Weather6")] ),

numPrimary $=3$ )

\#\#Output the summary details of the dataframe

summary(spotUMF)

\#\#Test models for detection covariates

fm1 $<$ - $\operatorname{colext}(\sim$ gravel1+distUP, 1, 1, date+air+water+year+Wind+Weather,spotUMF)

fm $2<-$ colext( $\sim$ gravel1+distUP, $1, \sim 1, \sim$ date+year,spotUMF)

fm3 $<-$ colext( $\sim$ gravel1+distUP, 1, 1, air+year,spotUMF)

fm $4<-$ colext $(\sim$ gravel1+distUP, 1, 1, $\sim$ water+year,spotUMF)

fm5 $<$ - colext( $\sim$ gravel1+distUP, 1, 1, $\sim$ year,spotUMF)

fm6 $<$ - $\operatorname{colext}(\sim$ gravel1+distUP, 1, 1, Wind+year,spotUMF)

$\mathrm{fm} 7<-$ colext $(\sim \operatorname{gravel} 1+$ distUP, 1, 1, Weather+year,spotUMF $)$

fm8 <- colext( gravel1+distUP, 1, 1, air+water+year+Wind+Weather,spotUMF)

fm9 <- colext( $\sim$ gravel1+distUP, 1, 1, date+water+year+Wind+Weather,spotUMF)

fm10 <- colext( $\sim$ gravel1+distUP, 1, 1, date+air+year+Wind+Weather,spotUMF)

fm11<- colext( gravel1+distUP, 1, 1, date+air+water+year+Wind,spotUMF)

fm1 $2<$ - colext( $\sim$ gravel1+distUP, $\sim 1, \sim 1, \sim$ date+air+water+year+Weather,spotUMF)

fm13<- $\operatorname{colext}(\sim 1, \sim 1, \sim 1, \sim 1, \operatorname{spotUMF})$

RABO.det $<$ - fitList('Global' $=$ fm1,

'Date' $=\mathrm{fm} 2$,

'Air' = fm3,

'Water' $=\mathrm{fm} 4$,

'Year' $=\mathrm{fm} 5$,

'Wind' $=$ fm6,

'Weather' $=\mathrm{fm} 7$,

'Global-Date' $=\mathrm{fm} 8$,

'Global-Air' = fm9,

'Global-Water' $=$ fm10,

'Global-Wind' $=$ fm11,

'Global-Weather' $=\mathrm{fm} 12$,

'Null' = fm13

)

\#Compare AIC value for the models

ms $1<$-modSel(RABO.det)

\#Output the model comparison results

toExport <- as(ms1, "data.frame") \# Everything

write.csv(toExport,file="RABOColExtInitPsi.csv")

\#\#Using detection covariates based on the highest performing model from above,

\#\#run models for the extinction covariates, including the global,

\#\#null, constant, and individual models for each covariate 
fm14 <- $\operatorname{colext}(\sim 1, \sim 1, \sim 1, \sim 1, \operatorname{spotUMF})$

fm1 $5<$ - colext( $\sim$ gravel1+distUP, $\sim 1, \sim 1, \sim$ year,spotUMF)

fm16<- colext( $\sim$ gravel1+distUP, 1, gravel+distUP+pctmc, $\sim$ year,spotUMF)

fm1 $7<$ - colext( $\sim$ gravel1+distUP, 1, gravel, $\sim$ year,spotUMF)

fm18<- colext( gravel1+distUP, 1, distUP, $\sim$ year,spotUMF)

fm19<- colext( $\sim$ gravel1+distUP, $1, \sim$ pctmc, $\sim$ year,spotUMF)

RABO.ext $<$ - fitList('null' $=$ fm14,

'constant' $=$ fm15,

'global' $=$ fm16,

'gravel' $=$ fm17,

'distTrib' = fm18,

'pctmc' $=$ fm19)

\#Compare AIC value for the models

ms $2<-\operatorname{modSel}($ RABO.ext)

\#Output the model comparison results

toExport <- as(ms2, "data.frame") \# Everything

write.csv(toExport,file="RABOColExtSummary.csv")

\#Output model results based on the model with the highest AICc from above

\#Estimates for initial psi

Est.Psi<-predict(fm19,type $=$ 'psi', data $=$ spotUMF $)$

write.csv(Est.Psi,file="RABOColExtPredictPsi.csv")

\#Estimates for extinction rates

E.ext $<$-predict $(\mathrm{fm} 19$, type $=$ 'ext')

write.csv(E.ext,file="RABOColExtPredictExt.csv")

\#Estimates for colonization rates

E.col<-predict $\left(\mathrm{fm} 19\right.$, type $=$ ' col' $\left.^{\prime}\right)$

write.csv(E.col,file="RABOColExtPredictCol.csv")

\#Detection probabilities

E.det $<$-predict(fm19, type $=$ 'det')

write.csv(E.det,file="RABOColExtPredictDet.csv")

\#\#Derive occupancy estimates

fm19<-nonparboot $(\mathrm{fm} 19, \mathrm{~B}=100)$

cbind(projected=projected(fm19)[2,],SE=fm19@projected.mean.bsse[2,]) 


\section{Appendix M. R Script for Western Pond Turtle Occupancy Model}

\#

\# Script for conducting a multi-year occupancy model using the package unmarked

\# for Trinity River basking western pond turtles.

\# This program was used to produce the estimates in the report:

\# Snover, M.L., and Adams, M.J., 2016, Herpetological monitoring and assessment on the Trinity River, Trinity

Country, California—Final report: U.S. Geological Survey Open-File Report 2016-1089, 93 p.

\# See report for a description of the variables.

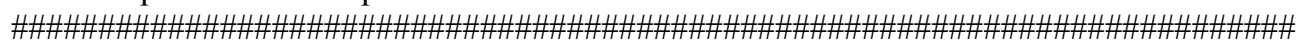

\#\#Load the libraries needed for this analysis

library(unmarked)

\#library(MuMIn)

\#\#Set the working directory for the location of the input files, this will need to be changed to run setwd( "C:/Users/Melissa/USGS_C_Drive/R_Work/WPTAbundance")

\#\#Read in the data, observations and covariates

SurveyData<-read.csv(file="WPT_Occu.csv",head=TRUE,sep=",")

\#\# Tell $\mathrm{R}$ what the observations are (1/0 for detected or not detected at each survey)

baskers.y<-SurveyData[,c("y.1","y.2","y.3","y.4","y.5","y.6","y.7","y.8","y.9")]

\#Standarize the continuous observation (survey-specific) covariates

DATE $<$ - as.matrix(SurveyData[,10:18])

$\#$ y.cross $<-$ as.matrix(SurveyData[,5:31])

\#y.cross[is.na(DATE) != is.na(y.cross)] <- NA

sd.DATE $<-\operatorname{sd}(\mathrm{c}(\mathrm{DATE})$, na.rm=TRUE)

mean.DATE $<-$ mean(DATE, na.rm=TRUE)

DATE <- (DATE - mean.DATE) / sd.DATE

Time <- as.matrix(SurveyData[,19:27])

sd.Time $<-$ sd(c(Time), na.rm=TRUE)

mean. Time $<-$ mean(Time, na.rm=TRUE)

Time $<$ - (Time - mean.Time) / sd.Time

Water <- as.matrix(SurveyData[,28:36])

sd. Water $<-$ sd(c(Water), na.rm=TRUE)

mean. Water $<-$ mean (Water, na.rm $=$ TRUE)

Water $<$ - (Water - mean.Water) / sd.Water

Air $<$ - as.matrix(SurveyData[,37:45])

sd.Air $<-\operatorname{sd}(\mathrm{c}($ Air), na.rm=TRUE)

mean.Air $<$ - mean(Air, na.rm=TRUE)

Air $<-$ (Air - mean.Air) / sd.Air

\#Standardize the Site-specific Covariates

Dist<-as.matrix(SurveyData[,74])

sd.Dist $<-\operatorname{sd}(\mathrm{c}$ (Dist), na.rm=TRUE)

mean.Dist $<$ - mean(Dist, na.rm=TRUE)

Dist $<-($ Dist - mean.Dist $) /$ sd.Dist

\#Yearly site covariates

VegR.z<-(SurveyData\$VegetationR)

VegL.z<-(SurveyData\$VegetationL) 


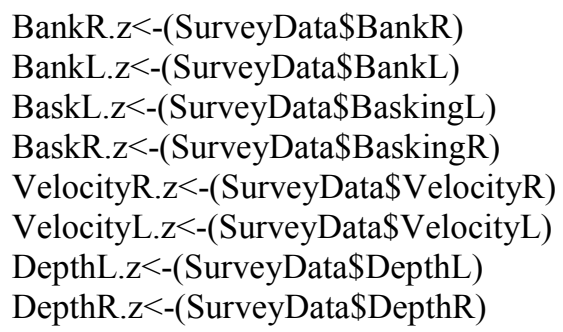

\#Define the years

years $<-$ as.character(2013:2015)

years $<-$ matrix (years, nrow(SurveyData), 3, byrow=TRUE)

\#Create the unmarked dataframe for a multi-year occupancy analysis

spotUMF $<$-unmarkedMultFrame $(y=$ baskers. $y$,

siteCovs $=$ data.frame(vegR=VegR.z, vegL $=$ VegL.z,bankR=BankR.z,bankL=BankL.z,

baskL=BaskL.z,baskR=BaskR.z,velR=VelocityR.z, velL=VelocityL.z,depthR=DepthR.z,

depthL=DepthL.z,distD=Dist),

yearlySiteCovs=list(year=years),

obsCovs $=$ list $($ date $=$ DATE, time $=$ Time, water $=$ Water,

air=Air,Wind=SurveyData[,c("Wind1","Wind2","Wind3","Wind4","Wind5","Wind6","Wind7","Wind8","Wind9")]

,Weather=SurveyData[,c("Weather1","Weather2","Weather3","Weather4","Weather5","Weather6","Weather7","We ather8","Weather9")] ),

numPrimary $=3$ )

\#\#Output the summary details of the dataframe

summary(spotUMF)

\#\#Test models for detection covariates

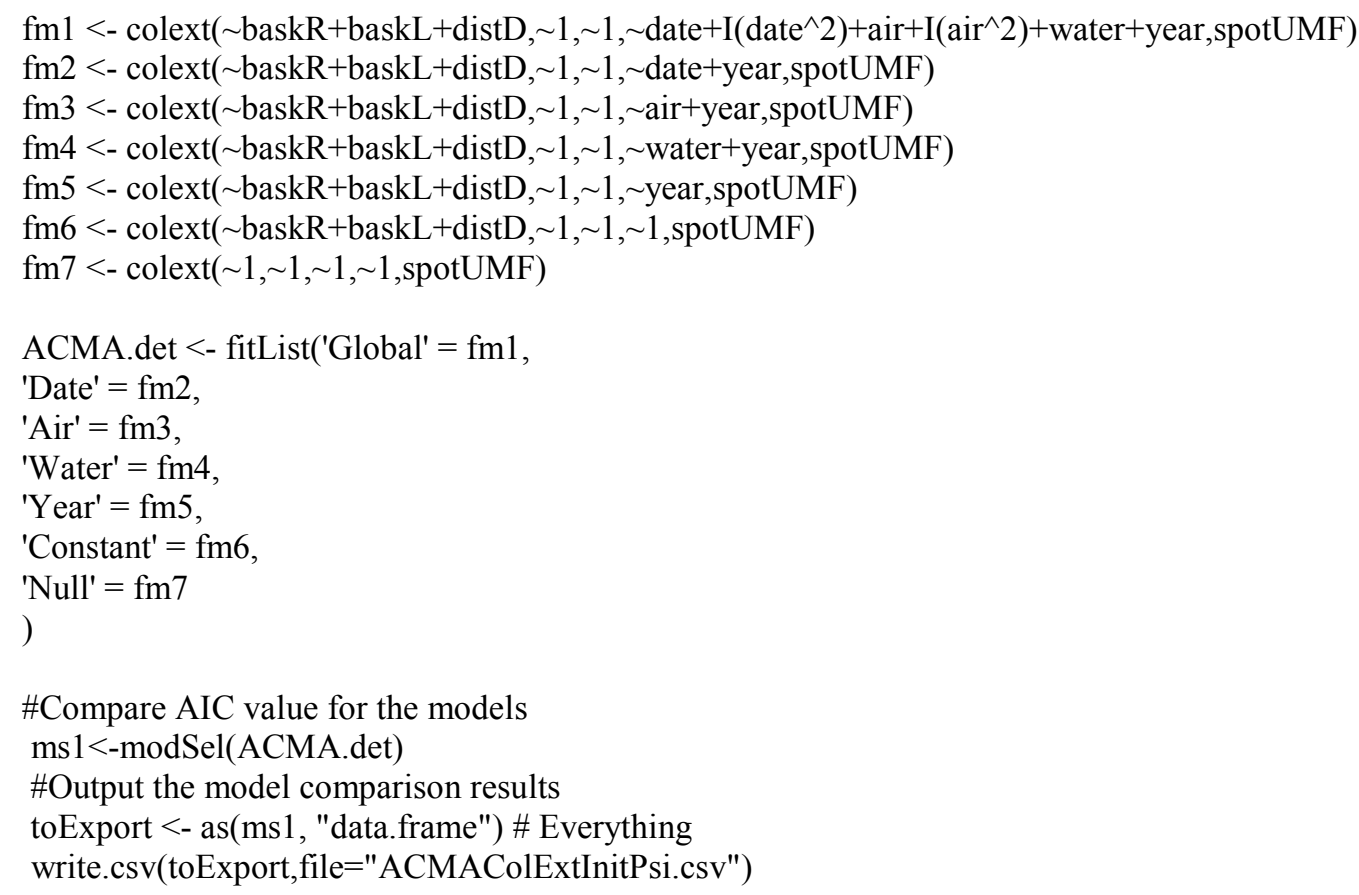

\#Compare AIC value for the models ms $1<-\operatorname{modSel}($ ACMA.det) \#Output the model comparison results toExport <- as(ms1, "data.frame") \# Everything write.csv(toExport,file="ACMAColExtInitPsi.csv")

\#\#Using detection covariates based on the highest performing model from above, 
\#\#run models for the extinction covariates, including the global,

\#\#null, constant, and individual models for each covariate

fm8 $<-\operatorname{colext}(\sim 1, \sim 1, \sim 1, \sim 1, \operatorname{spotUMF})$

fm9 $<-$

colext $\left(\sim\right.$ baskR+baskL+distD $, \sim 1, \sim 1, \sim$ bankR+bankL+baskR+baskL+velR + velL + depthR + depthL + distD + date $+\mathrm{I}\left(\right.$ date ${ }^{\wedge}$

2) + air $+\mathrm{I}\left(\right.$ air $\left.^{\wedge} 2\right)+$ water+year,spotUMF)

fm10 $<$ -

$\operatorname{colext}(\sim$ baskR+baskL+distD $, \sim 1, \sim$ bankR+bankL+baskR+baskL+velR +velL +distD $\sim$ bankR+bankL + baskR + baskL $+v$ elR+velL+distD+date $+\mathrm{I}\left(\right.$ date $\left.^{\wedge} 2\right)+$ air $+\mathrm{I}\left(\right.$ air $\left.^{\wedge} 2\right)+$ water + year,spotUMF $)$

fm1 $1<-$

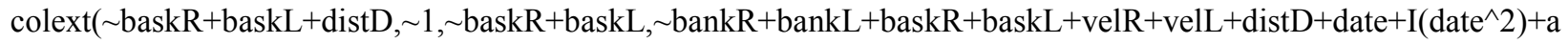
$\operatorname{ir}+\mathrm{I}\left(\operatorname{air}^{\wedge} 2\right)+$ water+year,spotUMF $)$

fm12<-

$\operatorname{colext}\left(\sim\right.$ baskR+baskL+distD $, \sim 1, \sim$ bankR+bankL, $\sim$ bankR+bankL+baskR+baskL+velR + velL + distD + date $+\mathrm{I}\left(\right.$ date $\left.{ }^{\wedge}\right)+$ air+I(air^2)+water+year,spotUMF)

fm13<-

$\operatorname{colext}\left(\sim\right.$ baskR+baskL+distD $, \sim 1, \sim$ velR + velL, $\sim$ bankR+bankL+baskR+baskL+velR + velL + distD + date $+\mathrm{I}\left(\right.$ date $\left.{ }^{\wedge} 2\right)+$ air + $\mathrm{I}\left(\right.$ air $\left.^{\wedge} 2\right)+$ water+year,spotUMF $)$

fm14 <-

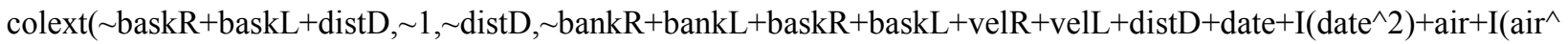
2)+water+year,spotUMF)

ACMA.ext <- fitList('null' $=$ fm8, 'constant' $=$ fm9,

'global' = fm10,

'bask' $=$ fm11,

'bank' = fm12,

'velocity' = fm13,

'distance'=fm14)

\#Compare AIC value for the models

ms $2<-$ modSel(ACMA.ext)

\#Output the model comparison results

toExport <- as(ms2, "data.frame") \# Everything

write.csv(toExport,file="ACMAColExtSummary.csv")

\#Output model results based on the model with the highest AICc from above

\#Estimates for initial psi

Est.Psi<-predict(fm9,type='psi',data=spotUMF)

write.csv(Est.Psi,file="ACMAColExtPredictPsi.csv")

\#Estimates for extinction rates

E.ext $<$-predict $\left(\right.$ fm9, type $={ }^{\prime}$ 'ext')

write.csv(E.ext,file="ACMAColExtPredictExt.csv")

\#Estimates for colonization rates

E.col<-predict $(\mathrm{fm} 9$, type $=$ 'col')

write.csv(E.col,file="ACMAColExtPredictCol.csv")

\#Detection probabilities

E.det $<$-predict $(\mathrm{fm} 9$, type $=$ 'det' $)$ 
write.csv(E.det,file="ACMAColExtPredictDet.csv")

\#\#Derive occupancy estimates

fm9<-nonparboot(fm9,B=100)

cbind(projected=projected(fm9)[2,],SE=fm9@projected.mean.bsse[2,]) 


\section{Appendix N. R Script for Western Pond Turtle Abundance Model}

\#

\# Script for conducting a multi-year occupancy model using the package unmarked

\# for Trinity River basking western pond turtles.

\# This program was used to produce the estimates in the report:

\# Snover, M.L., and Adams, M.J., 2016, Herpetological monitoring and assessment on the Trinity River,

Trinity Country, California—Final report: U.S. Geological Survey Open-File Report 2016-1089, 93 p.

\# See report for a description of the variables.

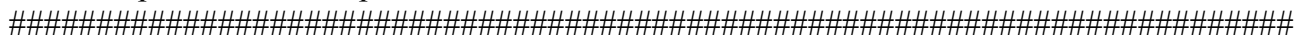

\#\#Load the libraries needed for this analysis

library(unmarked)

\#\#Set the working directory for the location of the input files, this will need to be changed to run

setwd( "C:/Users/Melissa/USGS_C_Drive/R_Work/WPTAbundance");

\#\#Read in the data, observations and covariates

SurveyData<-read.csv(file="WPT_Abund_2013_2015HML.csv",head=TRUE,sep=",")

\#\# Tell $\mathrm{R}$ what the observations are (1/0 for detected or not detected at each survey)

baskers.y<-SurveyData[,c("y.1","y.2","y.3","y.4","y.5","y.6","y.7","y.8","y.9")]

\#Standarize the continuous observation (survey-specific) covariates

DATE $<$ - as.matrix(SurveyData[,10:18])

sd.DATE $<-\operatorname{sd}(\mathrm{c}(\mathrm{DATE})$, na.rm=TRUE)

mean.DATE $<-$ mean(DATE, na.rm=TRUE)

DATE $<-($ DATE - mean.DATE) / sd.DATE

Time $<$ - as.matrix(SurveyData[,19:27])

sd.Time $<-\operatorname{sd}(\mathrm{c}($ Time $)$, na.rm=TRUE)

mean. Time $<-$ mean(Time, na.rm=TRUE)

Time $<-$ (Time - mean.Time) / sd.Time

Water $<$ - as.matrix(SurveyData[,28:36])

sd.Water $<-$ sd(c(Water), na.rm=TRUE)

mean. Water $<-$ mean(Water, na.rm=TRUE)

Water $<$ - (Water - mean.Water) / sd.Water

Air $<$ - as.matrix(SurveyData[,37:45])

sd.Air $<-$ sd(c(Air), na.rm=TRUE)

mean.Air $<$ - mean(Air, na.rm=TRUE)

Air $<$ - (Air - mean.Air) / sd.Air

\#Standardize the Site-specific Covariates

Dist<-as.matrix(SurveyData[,79])

sd.Dist $<-\operatorname{sd}(\mathrm{c}($ Dist $)$, na.rm=TRUE)

mean.Dist $<-$ mean(Dist, na.rm=TRUE)

Dist $<$ - (Dist - mean.Dist) / sd.Dist

\#Yearly site covariates

VegR.z<-(SurveyData\$VegetationR)

VegL.z $<-($ SurveyData\$VegetationL)

BankR.z<-(SurveyData\$BankR)

BankL.z<-(SurveyData\$BankL)

BaskL.z<-(SurveyData\$BaskingL)

BaskR.z<-(SurveyData\$BaskingR)

VelocityR.z<-(SurveyData\$VelocityR)

VelocityL.z<-(SurveyData\$VelocityL) 
DepthL.z<-(SurveyData\$DepthL)
DepthR.z<-(SurveyData\$DepthR)

\#Define the years

years $<-$ as.character $(2013: 2015)$

years $<-$ matrix (years, nrow(SurveyData), 3, byrow=TRUE)

\#Create the unmarked dataframe for a multi-year open population abundance analysis spotUMF $<$-unmarkedFramePCO(y=baskers.y, siteCovs $=$ data.frame(vegR $=$ VegR.z, vegL $=$ VegL.z,bankR=BankR.z,bankL=BankL.z, baskL=BaskL.z,baskR=BaskR.z,velR=VelocityR.z, velL=VelocityL.z,depthR=DepthR.z, $\operatorname{depthL}=$ DepthL.z,distD $=$ Dist $)$, yearlySiteCovs=list(year=years), obsCovs $=$ list $($ date $=$ DATE, time $=$ Time, water $=$ Water, air=Air,Wind=SurveyData[,c("Wind1","Wind2","Wind3","Wind4","Wind5","Wind6","Wind7","Wind8","Wind9")] ,Weather=SurveyData[,c("Weather1","Weather2","Weather3","Weather4","Weather5","Weather6","Weather7","We ather8","Weather9")] ),

numPrimary $=3$ )

\#Output the summary details of the dataframe

summary(spotUMF)

\#Test Models

fm1<-pcountOpen $(\sim$ baskL+baskR, $\sim 1, \sim 1$,

$\sim \mathrm{I}\left(\right.$ date $\left.^{\wedge} 2\right)+$ date $+\mathrm{I}\left(\mathrm{air}^{\wedge} 2\right)+$ air+water+year,data=spotUMF, mixture=c("NB"),K=50, dynamics="trend",se=TRUE) fm2<-pcountOpen $(\sim 1, \sim 1, \sim 1$,

$\sim \mathrm{I}\left(\right.$ date $\left.^{\wedge} 2\right)+$ date $+\mathrm{I}\left(\right.$ air^$\left.^{\wedge} 2\right)+$ air+water+year,data $=$ spotUMF, mixture=c("NB"),K=50, dynamics="trend",se=TRUE) fm3<-pcountOpen $(\sim 1, \sim 1, \sim 1$,

$\sim \mathrm{I}\left(\right.$ date $\left.^{\wedge} 2\right)+$ date $+\mathrm{I}\left(\operatorname{air}^{\wedge} 2\right)+$ air + water+year,data $=$ spotUMF, mixture $=\mathrm{c}(" \mathrm{NB} "), \mathrm{K}=50$, dynamics="notrend",se=TRUE)

WPT.abund<-fitList("lam(bask),gam(.),O(.),p(g)T"=fm1,

"lam(.),gam(.), $\mathrm{O}(),. \mathrm{p}(\mathrm{g}) \mathrm{T} "=\mathrm{fm} 2$,

"lam(.),gam(.),O(.),p(g)NT"=fm3)

ms $1<-\operatorname{modSel}($ WPT.abund)

toExport <- as(ms1, "data.frame") \# Everything

write.csv(toExport,file="WPTOpenCountSummary.csv")

\#Output population growth rate

$($ Est.Gamma<-predict(fm1,type='gamma',data=spotUMF) 
Publishing support provided by the U.S. Geological Survey Science Publishing Network, Tacoma Publishing Service Center

For more information concerning the research in this report, contact the Director, Forest and Rangeland Ecosystem Science Center U.S. Geological Survey

777 NW 9th St., Suite 400

Corvallis, Oregon 97330

http://fresc.usgs.gov/ 
